\title{
Boundary critical behavior at $m$-axial Lifshitz points for a boundary plane parallel to the modulation axes
}

\author{
H. W. Diehl, A. Gerwinski, and S. Rutkevich* \\ Fachbereich Physik, Universität Duisburg-Essen, 45117 Essen, Germany
}

(Dated: November 21, 2018)

\begin{abstract}
The critical behavior of semi-infinite $d$-dimensional systems with $n$-component order parameter $\phi$ and short-range interactions is investigated at an $m$-axial bulk Lifshitz point whose wave-vector instability is isotropic in an $m$-dimensional subspace of $\mathbb{R}^{d}$. The associated $m$ modulation axes are presumed to be parallel to the surface, where $0 \leq m \leq d-1$. An appropriate semi-infinite $|\phi|^{4}$ model representing the corresponding universality classes of surface critical behavior is introduced. It is shown that the usual $O(n)$ symmetric boundary term $\propto \phi^{2}$ of the Hamiltonian must be supplemented by one of the form $\dot{\lambda} \sum_{\alpha=1}^{m}\left(\partial \phi / \partial x_{\alpha}\right)^{2}$ involving a dimensionless (renormalized) coupling constant $\lambda$. The implied boundary conditions are given, and the general form of the field-theoretic renormalization of the model below the upper critical dimension $d^{*}(m)=4+m / 2$ is clarified. Fixed points describing the ordinary, special, and extraordinary transitions are identified and shown to be located at a nontrivial value $\lambda^{*}$ if $\epsilon \equiv d^{*}(m)-d>0$. The surface critical exponents of the ordinary transition are determined to second order in $\epsilon$. Extrapolations of these $\epsilon$ expansions yield values of these exponents for $d=3$ in good agreement with recent Monte Carlo results for the case of a uniaxial $(m=1)$ Lifshitz point. The scaling dimension of the surface energy density is shown to be given exactly by $d+m(\theta-1)$, where $\theta=\nu_{l 4} / \nu_{l 2}$ is the anisotropy exponent.
\end{abstract}

PACS numbers: 75.10.Hk, 68.35.Rh, 64.60.Ht, 05.70.Jk

Keywords: surface critical behavior, Lifshitz points, scaling, field theory

\section{INTRODUCTION}

As is well known, there are many physical systems whose phase diagrams exhibit a Lifshitz point. ${ }^{1-4}$ A Lifshitz point is a multicritical point at which a disordered, a homogeneous ordered, and a modulated ordered phase meet. It divides the critical line that separates the disordered phase from the two ordered ones into two sections, and marks the onset of a wave-vector instability. In the case of an $m$-axial Lifshitz point this instability occurs in an $m$-dimensional subspace of $\mathbb{R}^{d}$. Prominent examples of systems with uniaxial $(m=1)$ Lifshitz points are, on the experimental side, the metallic compound ${ }^{5-8} \mathrm{MnP}$, and on the theoretical side, the three-dimensional axial next-nearest-neighbor Ising (ANNNI) model. ${ }^{9,10}$

Although the notion of a Lifshitz point was introduced decades ago, ${ }^{11}$ the successful application of modern field theory tools to the study of critical behavior at such points is a fairly recent development. ${ }^{12-17}$ A full twoloop renormalization group analysis and $\epsilon$ expansion of all critical exponents to second order in $\epsilon$ about the upper critical dimension $d^{*}(m)=4+m / 2(0 \leq m \leq 8)$ has been accomplished for general values of $m$ only two years ago. ${ }^{14,15}$

In the present paper we wish to study the effects of surfaces on the critical behavior at an $m$-axial Lifshitz point. Previous work on this problem is scarce. It started in 1986 with Gumbs' investigation ${ }^{18}$ based on Landau theory. This was reconsidered, completed, and partly corrected by Binder and Frisch. ${ }^{19}$ In either one of these papers, only the uniaxial case $m=1$ was considered, and the special axis along which ferromagnetic nearest-neighbor and antiferromagnetic next- nearest-neighbor interactions compete was chosen perpendicular to the surface plane. The complementary case in which the special axis of the considered semi-infinite ANNNI model is parallel to the surface was dealt with on the level of mean field theory in subsequent work by Frisch et al. ${ }^{20}$ Even more recently, Pleimling ${ }^{21}$ presented results of Monte Carlo simulations of semi-infinite ANNNI models for both types of surface orientations.

A characteristic feature of critical behavior at Lifshitz points is anisotropic scale invariance. Let $\Delta x_{\alpha}$ and $\Delta x_{\beta}$ with $1 \leq \alpha \leq m$ and $m<\beta \leq d$ denote displacements along the corresponding Euclidean axes and suppose that the $\Delta x_{\beta}$ are rescaled by a factor $\ell$. Anisotropic scale invariance means that the $\Delta x_{\alpha}$ must be rescaled by a nontrivial power $\ell^{\theta}$ in order that the system looks selfsimilar on different scales. ${ }^{22}$

An obvious consequence of this property is that two distinct cases of surface orientations must be distinguished when dealing with surface critical behavior at bulk Lifshitz points: a parallel one for which the surface normal $\boldsymbol{n}$ is along a $\beta$-direction (all $\alpha$-directions being parallel to the surface plane), and a perpendicular one for which $\boldsymbol{n}$ is along an $\alpha$-direction. Since the distance $z$ from the surface scales differently in these two cases, one expects (i) that the respective values of the surface exponents are in general different, even in mean-field theory, and (ii) that the boundary terms which must be included in the Hamiltonian are different as well. Expectation (i) is borne out by both the mean-field results of Refs. 19 and 20 as well as by Pleimling's Monte Carlo results; ${ }^{21}$ (ii) turns out to be equally true. ${ }^{23}$

In this paper ${ }^{24,25}$ we will focus our attention on the case of parallel surface orientation, leaving the study of 
the other, "perpendicular" case to a subsequent paper [which will confirm the above conclusion (ii)]. To our knowledge, experimental investigations of even bulk critical behavior at Lifshitz points $5^{5,8}$ have so far been restricted to the uniaxial case $m=1$, and this is also still the only one for which Monte Carlo simulation results on surface critical behavior at bulk Lifshitz points are available. ${ }^{21}$

Although $m=1$ thus appears to be the case of greatest interest, we will keep the value of $m$ as general as possible, imposing at this stage no restriction other than the obvious one $0 \leq m \leq d-1 .{ }^{26}$ Just as in essentially all renormalization group (RG) analyses of bulk critical behavior with $m>1$, we will make, however, the simplifying assumption that the wave-vector instability is isotropic in the $m$-dimensional subspace of $\alpha$ directions. Let us nevertheless add a cautionary remark about this presumed " $m$-isotropy". In a recent study of bulk critical behavior, ${ }^{27}$ this assumption has been relaxed by requiring only invariance in this subspace under the cubic, or even a lower, symmetry group. The results indicate that the isotropic bulk fixed point for $m \geq 2$ gets destabilized by bulk terms of second order in $\phi$ and fourth order in the derivatives $\partial / \partial x_{\alpha}$, although the associated crossover exponent appears to be fairly small. The inclusion of such non-isotropic terms is beyond the scope of the present work and, of course, meaningless in the uniaxial case $m=1$.

The remainder of this paper is organized as follows. In the next section we specify the Hamiltonian representing the corresponding universality classes for surface critical behavior at $m$-axial bulk Lifshitz points. We explain which boundary terms must be included in it and give the implied boundary conditions. In Sec. III we first clarify the renormalization of the model for general values of its parameters and derive the resulting RG equations. On the basis of two-loop results, we then discuss the form of the RG flow in the space of the surface interaction constants and identify the fixed points describing the ordinary, special, and extraordinary transitions.

Section IV deals specifically with the ordinary transition. We show that the RG analysis can be simplified in much the same way as in the $m=0$ case of a critical point ${ }^{28}$ by choosing Dirichlet boundary conditions, considering correlation functions involving the normal derivative of the order parameter at the boundary, and making use of the boundary operator expansion. The required RG functions are evaluated to two-loop order; the scaling index of the surface energy density is determined exactly.

In Sec. $\mathrm{V}$ results to order $\epsilon^{2}$ are given for the critical exponents of the ordinary transition. These $\epsilon$ expansions are exploited to estimate the values of these exponents for the uniaxial, one-component case $m=n=1$ in three dimensions. Section VI contains a brief summary and concluding remarks. Finally, there are five appendixes, explaining details of our calculations.

\section{THE HAMILTONIAN, ITS BOUNDARY TERMS, AND IMPLIED BOUNDARY CONDITIONS}

We consider systems with short-range interactions, assuming also that the perturbations of these interactions induced by the presence of the surface decay to zero within a short distance from it. With these assumptions the Hamiltonian can be taken to be of the form

$$
\mathcal{H}=\int_{\mathfrak{V}} \mathcal{L}_{\mathrm{b}}(\boldsymbol{x}) d V+\int_{\mathfrak{B}} \mathcal{L}_{1}(\boldsymbol{x}) d A,
$$

where $\mathcal{L}_{\mathrm{b}}(\boldsymbol{x})$ and $\mathcal{L}_{1}(\boldsymbol{x})$ depend on the order parameter density $\boldsymbol{\phi}(\boldsymbol{x})=\left(\phi_{a}(\boldsymbol{x}), a=1, \ldots, n\right)$ and its spatial derivatives up to a finite order. Here the volume and surface integrals extend over $\mathfrak{V}$, the $d$-dimensional half-space $\mathbb{R}_{+}^{d}=\mathbb{R}^{d-1} \times[0, \infty)$, and $\mathfrak{B}$, the $d-1$ dimensional surface plane $z=0$, respectively. Writing $\boldsymbol{x}=\left(\left(x_{\alpha}\right),\left(x_{\beta}\right)\right)=(\boldsymbol{r}, z)$, we split the position vector $\boldsymbol{x}$ into its $m$-dimensional component $\left(x_{\alpha}\right) \in \mathbb{R}^{m}$ and $(d-m)$-dimensional one $\left(x_{\beta}\right)=\left(\left(r_{\beta}\right), z\right)$, where $\boldsymbol{r}=\left(\left(r_{\alpha}\right) \equiv\left(x_{\alpha}\right),\left(r_{\beta}\right)\right)$ is the $(d-1)$-dimensional coordinate along the surface. We choose the same bulk density as in Refs. 14-17, namely

$$
\begin{aligned}
\mathcal{L}_{\mathrm{b}}(\boldsymbol{x})= & \frac{\stackrel{\circ}{2}}{2}\left(\sum_{\alpha=1}^{m} \partial_{\alpha}^{2} \phi\right)^{2}+\frac{1}{2} \sum_{\beta=m+1}^{d}\left(\partial_{\beta} \phi\right)^{2} \\
& +\frac{\stackrel{\rho}{2}}{2} \sum_{\alpha=1}^{m}\left(\partial_{\alpha} \phi\right)^{2}+\frac{\stackrel{\circ}{\tau}}{2} \phi^{2}+\frac{\stackrel{\circ}{u}}{4 !}|\phi|^{4},
\end{aligned}
$$

where $\partial_{\alpha}$ and $\partial_{\beta}$ denote the spatial derivatives $\partial / \partial x_{\alpha}$ and $\partial / \partial x_{\beta}$ with $1 \leq \alpha \leq m$ and $m+1 \leq \beta \leq d$, respectively. Had we not assumed that the wave-vector instability is isotropic in the subspace $\mathbb{R}^{m}$, the term $\left(\sum_{\alpha=1}^{m} \partial_{\alpha}^{2} \phi\right)^{2}$ would have to be supplemented by similar, albeit less symmetric, terms involving four derivatives, such $a^{27}$ $\sum_{\alpha=1}^{m}\left(\partial_{\alpha}^{2} \phi\right)^{2}$.

In order to decide which monomials should be included in $\mathcal{L}_{1}(\boldsymbol{x})$, we use power counting. Recalling from Ref. 14 the naive dimensions $\left[x_{\beta}\right]=[z]=\mu^{-1},\left[x_{\alpha}\right]=\dot{\sigma}^{1 / 4} \mu^{-1 / 2}$, and $[\phi(\boldsymbol{x})]=\stackrel{\circ}{\sigma}^{-m / 8} \mu^{(d-2-m / 2) / 2}$ (where $\mu$ is an arbitrary momentum scale), we include only such $O(n)$ invariant monomials whose interaction constants have nonnegative $\mu$-dimensions at the upper critical dimension $d^{*}(m)$. It is not difficult to see that the choice

$$
\mathcal{L}_{1}(\boldsymbol{x})=\frac{\stackrel{\circ}{c}}{2} \phi^{2}+\frac{\AA}{2} \sum_{\alpha=1}^{m}\left(\partial_{\alpha} \phi\right)^{2}
$$

is sufficient since the two additional candidates $\phi \partial_{\alpha} \partial_{\alpha} \phi$ and $\phi \partial_{n} \phi$ both may be dropped. The integral $\int_{\mathfrak{B}}$ of the former reduces to that of the derivative term retained in Eq. (3) upon integration by parts, and the latter can be shown to be redundant in much the same way as in the familiar case of the standard semi-infinite $\phi^{4}$ model. ${ }^{28}$ One 
must merely note that the action defined by Eqs. (1)-(3) leads to the boundary condition ${ }^{28-31}$

$$
\partial_{n} \phi(\boldsymbol{x})=\left(\stackrel{\circ}{c}-\stackrel{\circ}{\lambda} \partial_{\alpha} \partial_{\alpha}\right) \boldsymbol{\phi}(\boldsymbol{x}), \quad \boldsymbol{x} \in \mathfrak{B},
$$

which in turn implies that the surface term $\int_{\mathfrak{B}} \phi \partial_{n} \phi$ is equivalent to a linear combination of the two monomials present in $\mathcal{L}_{1}$. Here a sum convention which will frequently be employed below has been introduced: Pairs of equal $\alpha$ and $\beta$ indices are to be summed over $\alpha=1, \ldots, m$ and $\beta=m+1, \ldots, d$, respectively.

We need the free propagator of the disordered phase, $G\left(\boldsymbol{x}, \boldsymbol{x}^{\prime}\right)=\left[\delta^{2} \mathcal{H} /\left.\delta \boldsymbol{\phi} \delta \phi\right|_{\phi=0}\right]^{-1}\left(\boldsymbol{x}, \boldsymbol{x}^{\prime}\right)$. This is a solution to the equation

$$
\left[\stackrel{\circ}{\sigma}\left(\partial_{\alpha} \partial_{\alpha}\right)^{2}-\stackrel{\circ}{\rho} \partial_{\alpha} \partial_{\alpha}-\partial_{\beta} \partial_{\beta}+\stackrel{\circ}{\tau}\right] G\left(\boldsymbol{x}, \boldsymbol{x}^{\prime}\right)=\delta\left(\boldsymbol{x}-\boldsymbol{x}^{\prime}\right),
$$

subject to the boundary condition

$$
\left(\partial_{n}-\stackrel{\imath}{c}+\grave{\lambda} \partial_{\alpha} \partial_{\alpha}\right) G\left(\boldsymbol{x}, \boldsymbol{x}^{\prime}\right)=0, \quad \boldsymbol{x} \in \mathfrak{B}, \quad \boldsymbol{x}^{\prime} \notin \mathfrak{B},
$$

where $\partial_{n}\left(\equiv \partial_{z}\right)$ denotes the derivative along the inward normal $\boldsymbol{n}$.

We take periodic boundary conditions along all $d-1$ axes parallel to the surface. Let $\boldsymbol{p}=\left(\left(p_{\alpha}\right),\left(p_{\beta}\right)\right) \in$ $\mathbb{R}^{m} \times \mathbb{R}^{d-m-1}$ be the momentum conjugate to $\boldsymbol{r}$, and $\hat{G}\left(\boldsymbol{p} ; z, z^{\prime}\right)$ be the corresponding Fourier transform of $G$ with respect to the $d-1$ coordinates parallel to the surface. In this $\boldsymbol{p} z$ representation $\hat{G}$ is easily evaluated; one finds

$$
\begin{aligned}
& \hat{G}\left(\boldsymbol{p} ; z, z^{\prime}\right)
\end{aligned}
$$

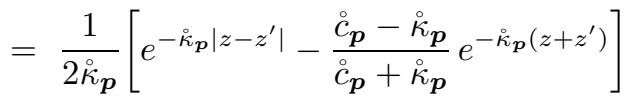

with

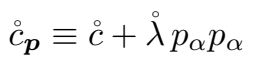

and

$$
\stackrel{\circ}{\kappa}_{\boldsymbol{p}}=\sqrt{\stackrel{\circ}{\tau}+\stackrel{\circ}{\rho} p_{\alpha} p_{\alpha}+p_{\beta} p_{\beta}+\stackrel{\circ}{\sigma}\left(p_{\alpha} p_{\alpha}\right)^{2}} .
$$

The part of $\hat{G}$ that depends on $\left|z-z^{\prime}\right|$ is its bulk ana$\log \hat{G}_{\mathrm{b}}$; the remainder is the contribution induced by the presence of the surface. Results for the free bulk propagator $G_{\mathrm{b}}\left(\boldsymbol{x}-\boldsymbol{x}^{\prime}\right)$ in position and momentum space can be found in Refs. 14 and 15. Specifically at the (Gaussian) Lifshitz point $\stackrel{\circ}{\tau}=\stackrel{\circ}{\rho}=0$, one has

$$
G_{\mathrm{b}}(\boldsymbol{x})=X^{-2+\epsilon} \stackrel{\circ}{\sigma}^{-m / 4} \Phi_{m, d}\left(\stackrel{\circ}{\sigma}^{-1 / 4} \check{x} X^{-1 / 2}\right)
$$

with

$$
X \equiv \sqrt{x_{\beta} x_{\beta}}, \quad \check{x} \equiv \sqrt{x_{\alpha} x_{\alpha}},
$$

and

$$
\begin{aligned}
\Phi_{m, d}(v)= & \frac{1}{2^{2+m} \pi^{(6+m-2 \epsilon) / 4}}\left[\frac{\Gamma\left(1-\frac{\epsilon}{2}\right)}{\Gamma\left(\frac{m+2}{4}\right)}\right. \\
& \times{ }_{1} F_{2}\left(1-\frac{\epsilon}{2} ; \frac{1}{2}, \frac{m+2}{4} ; \frac{v^{4}}{64}\right)-\frac{v^{2} \Gamma\left(\frac{3-\epsilon}{2}\right)}{4 \Gamma\left(1+\frac{m}{4}\right)} \\
& \left.\times{ }_{1} F_{2}\left(\frac{3-\epsilon}{2} ; \frac{3}{2}, 1+\frac{m}{4} ; \frac{v^{4}}{64}\right)\right],
\end{aligned}
$$

where $\epsilon=4+\frac{m}{2}-d$ while ${ }_{p} F_{q}$ means the generalized hypergeometric function.

In our calculations below we need, in particular, the free bulk propagator between a point $\boldsymbol{x}=(\boldsymbol{r}, z)$ and its mirror point $\check{\boldsymbol{x}} \equiv(\boldsymbol{r},-z)=\boldsymbol{x}-2 z \boldsymbol{n}$. With the aid of the Taylor expansion of the scaling function $\Phi_{m, d}(v)$ given in Eqs. (10) and (11) of Ref. 15 one easily obtains the result

$$
\begin{aligned}
G_{\mathrm{b}}(\boldsymbol{x}-\check{\boldsymbol{x}}) & =G_{\mathrm{b}}(2 z \boldsymbol{n})=\Phi_{m, d}(0) \circ^{-m / 4}(2 z)^{\epsilon-2} \\
& =F_{m, \epsilon} \frac{\Gamma(2-\epsilon) \sin (\epsilon \pi / 2)}{\epsilon \pi \dot{\sigma}^{m / 4}} z^{\epsilon-2}
\end{aligned}
$$

in which

$$
F_{m, \epsilon}=\frac{\Gamma(1+\epsilon / 2) \Gamma^{2}(1-\epsilon / 2) \Gamma(m / 4)}{(4 \pi)^{(8+m-2 \epsilon) / 4} \Gamma(2-\epsilon) \Gamma(m / 2)}
$$

is a factor introduced in Ref. 15; just as there, we will absorb it in the renormalized coupling constant $u$ to be defined below.

\section{RENORMALIZATION GROUP}

\section{A. General considerations}

Let us introduce the cumulants involving $N$ fields $\phi_{a_{j}}$ at points $\boldsymbol{x}_{j}$ off the surface and $M$ boundary fields $\phi_{b_{k}}^{\mathfrak{B}}\left(\boldsymbol{r}_{k}\right) \equiv \phi_{b_{k}}\left(\boldsymbol{r}_{k}, 0\right)$, namely

$$
G^{(N, M)}(\boldsymbol{x} ; \boldsymbol{r})=\left\langle\prod_{j=1}^{N} \phi_{a_{j}}\left(\boldsymbol{x}_{j}\right) \prod_{k=1}^{M} \phi_{b_{k}}^{\mathfrak{B}}\left(\boldsymbol{r}_{k}\right)\right\rangle^{\mathrm{cum}} .
$$

Here $\boldsymbol{x}$ and $\boldsymbol{r}$ are convenient short-hands for the sets of position variables $\left\{\boldsymbol{x}_{j}\right\}$ and $\left\{\boldsymbol{r}_{k}\right\}$, and the components indices $\left\{a_{j}\right\}$ and $\left\{b_{k}\right\}$ have been suppressed on the righthand side for the sake of notational simplicity.

We wish to analyze the critical behavior of these functions in bulk dimensions $d \leq d^{*}(m)$ using a field-theoretic RG approach and the $\epsilon=d^{*}(m)-d$ expansion. To regularize their ultraviolet (uv) singularities, we employ dimensional regularization. Aside from the bulk uv singularities induced by the bulk part of the free propagator, additional primitive ones localized on the surface occur.

Consider first the former "bulk uv singularities". In previous investigations ${ }^{14,15}$ of the bulk model, $\stackrel{\rho}{\circ}$ and $\stackrel{\sim}{\tau}$ were set to their values $\stackrel{\circ}{\mathrm{LP}}_{\mathrm{L}}$ and $\stackrel{\circ}{\mathrm{LP}}_{\mathrm{L}}$ at the Lifshitz point in the actual calculations, or else deviations $\delta \stackrel{\circ}{\equiv} \stackrel{\circ}{\tau}-\stackrel{\circ}{\tau}_{\mathrm{LP}} \neq 0$ with $\delta \stackrel{\circ}{\equiv} \stackrel{\circ}{\rho}-\stackrel{\circ}{\rho}_{\mathrm{LP}}=0$ and $\delta \stackrel{\circ}{\alpha} \neq 0$ with $\delta \stackrel{\rho}{=} 0$ were considered. This is sufficient to determine the two RG eigenexponents $1 / \nu_{l 2}$ and $\varphi / \nu_{l 2}$ associated with the corresponding eigenoperators at the infrared (ir) stable fixed point (where $\nu_{l 2}$ is a standard correlation exponent while $\varphi$ means the crossover exponent pertaining to $\delta \rho$ ). One must remember, however, that in the $\rho$ dependent scaling forms of the correlation functions derived in Refs. 14 and 15 the linear scaling fields associated 
with these eigenexponents ought to be replaced by nonlinear ones when considering general deviations $\delta \dot{\rho}$ and

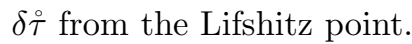

Here we wish to go beyond these previous analyses

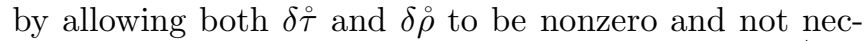
essarily small. Since the naive dimension of $\rho \circ^{-1 / 2}$ is $\left[\stackrel{\circ}{\circ}{ }^{-1 / 2}\right]=\mu$, we see that deviations $\delta \stackrel{\circ}{\rho} 0$ may give contributions to the renormalization of $\delta \stackrel{\circ}{\text {. }}$ That is, counterterms of the form $\propto \mu \rho^{2} \int_{\mathfrak{V}} \phi^{2}$ (where $\rho$ is the dimensionless renormalized counterpart of $\left.\delta \stackrel{\circ}{\circ}^{-1 / 2}\right)$ are possible. Combining this with the considerations made in Ref. 14 and 15, we can conclude that the bulk uv singularities can absorbed by making the following "bulk reparametrizations":

$$
\begin{aligned}
\phi & =Z_{\phi}^{1 / 2} \phi_{\text {ren }}, \\
\stackrel{\circ}{\sigma} & =Z_{\sigma} \sigma, \\
\stackrel{\circ}{\tau}-\stackrel{\circ}{\mathrm{LP}} & =\mu^{2} Z_{\tau}\left[\tau+A_{\tau} \rho^{2}\right], \\
(\stackrel{\circ}{\rho} \stackrel{\circ}{\mathrm{LP}})^{\circ} \stackrel{\circ}{\sigma}^{-1 / 2} & =\mu Z_{\rho} \rho, \\
\stackrel{\circ}{\mathrm{u}}^{-m / 4} F_{m, \epsilon} & =\mu^{\epsilon} Z_{u} u .
\end{aligned}
$$

Here $Z_{\iota}=Z_{\iota}(u, \epsilon), \iota=\sigma, \rho, u$ are bulk renormalization factors for which results to two-loop order were given in Refs. 14 and 15 for general values of $m$. In our perturbative approach based on dimensional regularization and

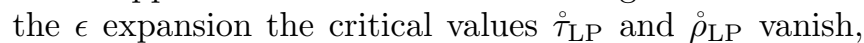
as usual. $A_{\tau}$ is the additional renormalization function associated with the counterterm discussed above. Owing to the restrictive assumptions made in Ref. 14 and 15, it was not needed - and hence not computed - there. A straightforward one-loop calculation yields

$$
A_{\tau}(u, \epsilon)=-\frac{n+2}{3} \frac{m}{16} \frac{u}{\epsilon}+O\left(u^{2}\right) .
$$

Next, we turn to the "surface uv singularities" induced by the free propagator's "surface part", i.e., the part proportional to $e^{-\aleph_{p}}\left|z-z^{\prime}\right|$. They require additional counterterms, localized on the surface. To determine their form, we use power counting in conjunction with what is generally known about such field theories with planar boundaries. ${ }^{28,32}$ In comparison to the usual $(m=0)$ semi-infinite $\phi^{4}$ model, a qualitative change occurs: There is a surface variable with zero $\mu$-dimension, namely $\stackrel{\circ}{\gamma}^{-1 / 2}$. This implies that the surface renormalization functions do not only depend on the renormalized bulk coupling constant $u$ but additionally on the renormalized analog of this bare variable, which we denote as $\lambda$. Furthermore, power counting suggests that a surface counterterm of the form $\propto \int_{\mathfrak{B}} d A \sum_{\alpha}\left(\partial_{\alpha} \phi\right)^{2}$ is needed even if $\dot{\lambda}$ is set to zero. If a large-momentum cutoff $\Lambda$ were used to regularize the uv singularities of the theory, then the associated renormalization function would diverge as $\ln \Lambda$. Hence it must have poles in $\epsilon$ in renormalization schemes based on dimensional regularization such as ours.
Finally, arguments completely analogous to those giving the contributions $\propto \rho^{2}$ to the renormalization of $\stackrel{\circ}{\tau}$ tell us that the renormalization of the surface enhancement variable $\stackrel{\circ}{c}$ involves contributions linear in $\rho$, and hence is not multiplicative. The upshot of these considerations is that the following reparametrizations of surface quantities are needed:

$$
\begin{aligned}
\phi^{\mathfrak{B}} & =\left(Z_{\phi} Z_{1}\right)^{1 / 2} \phi_{\text {ren }}^{\mathfrak{B}}, \\
\stackrel{\circ}{c}-\stackrel{\circ}{\mathrm{sp}}_{\mathrm{s}} & =\mu Z_{c}\left[c+A_{c}(u, \lambda, \epsilon) \rho\right], \\
\stackrel{\circ}{\circ}-1 / 2 & =\lambda+P_{\lambda}(u, \lambda, \epsilon) .
\end{aligned}
$$

Here the surface renormalization factors $Z_{1, c}=$ $Z_{1, c}(u, \lambda, \epsilon)$ have the form

$$
\begin{aligned}
Z_{1, c}-1 & =\sum_{i, j=1}^{\infty} Z_{1, c}^{(i,-j)}(\lambda) u^{i} \epsilon^{-j} \\
& =\sum_{i, j=1}^{\infty} \sum_{k=0}^{\infty} Z_{1, c}^{(i,-j ; k)} u^{i} \epsilon^{-j} \lambda^{k}
\end{aligned}
$$

provided we fix them by requiring that the poles be minimally subtracted. Likewise we have for the renormalization function $P_{\lambda}$,

$$
\begin{aligned}
P_{\lambda}(u, \lambda, \epsilon) & =\sum_{i, j=1}^{\infty} P_{\lambda}^{(i,-j)}(\lambda) u^{i} \epsilon^{-j} \\
& =\sum_{i, j=1}^{\infty} \sum_{k=0}^{\infty} P_{\lambda}^{(i,-j ; k)} u^{i} \epsilon^{-j} \lambda^{k}
\end{aligned}
$$

and similarly for $A_{c}$.

It is easy to see that the one-loop coefficient $P_{\lambda}^{(1,-1)}(\lambda)$ vanishes for $\lambda=0$. This follows from the fact that the tadpole graph $\bigcirc$ is independent of the momentum $\boldsymbol{p}$, so that no subtraction corresponding to a surface two-point counterterm $\propto \boldsymbol{p}_{\alpha}^{2}$ is required for the one-loop graph of $\left\langle\phi \phi^{\mathfrak{B}}\right\rangle$ if $\lambda=0$. On the other hand, a subtraction of this kind is needed for its two-loop subgraph with $\lambda=0$ (where the crossed circle marks the external surface point). Hence, $P_{\lambda}^{(2,-1)}(\lambda=0)$ does not vanish.

\section{B. One-loop results for general values of $\lambda$}

At one-loop order, the bulk renormalization factors $Z_{\phi}$, $Z_{\sigma}, Z_{\tau}, Z_{\rho}$, and $Z_{u}$ are known to be independent of $m$ and hence equal to their $m=0$ analogs. Obviously, this property cannot be expected to carry over to the surface renormalization factors $Z_{1}(u, \lambda, \epsilon)$ and $Z_{c}(u, \lambda, \epsilon)$ because of their $\lambda$ dependence. On the other hand, it does hold for $Z_{1}(u, 0, \epsilon)$ and $Z_{c}(u, 0, \epsilon)$.

To see this, note that a straightforward calculation presented in Appendix $\mathrm{C}$ yields the one-loop results

$$
Z_{1}(u, \lambda, \epsilon)=1+\frac{n+2}{3} \frac{i_{1}(\lambda ; m) u}{2 \epsilon}+O\left(u^{2}\right),
$$


$Z_{c}(u, \lambda, \epsilon)=1+\frac{n+2}{3} \frac{\left[2 i_{2}(\lambda ; m)-i_{1}(\lambda ; m)\right] u}{2 \epsilon}+O\left(u^{2}\right)$,

and

$$
P_{\lambda}(u, \lambda, \epsilon)=-\frac{n+2}{3} \frac{i_{1}(\lambda ; m) \lambda u}{2 \epsilon}+O\left(u^{2}\right) .
$$

Here $i_{1}(\lambda ; m) \equiv i_{1}(\lambda, 0 ; m)$ and $i_{2}(\lambda ; m) \equiv i_{2}(\lambda, 0 ; m)$ are special cases of the integrals

$$
i_{1}(\lambda, \epsilon ; m)=\int_{0}^{1} d t \frac{2 t^{(m-2) / 2}\left(1-t^{2}\right)^{(2-2 \epsilon-m) / 4}}{B[m / 4,(6-m-2 \epsilon) / 4]} \frac{1-\lambda t}{1+\lambda t}
$$

and

$$
\begin{aligned}
& i_{2}(\lambda, \epsilon ; m) \\
& \quad=\int_{0}^{1} d t \frac{2 t^{(m-2) / 2}\left(1-t^{2}\right)^{(2-2 \epsilon-m) / 4}}{B[m / 4,(6-m-2 \epsilon) / 4]} \frac{1}{(1+\lambda t)^{2}},
\end{aligned}
$$

where $B(a, b)$ is the Euler Beta function.

At $\lambda=0$ and for $\lambda \rightarrow \infty$ we have

$$
i_{1}(0 ; m)=i_{2}(0 ; m)=-i_{1}(\infty ; m)=1, \quad i_{2}(\infty ; m)=0 .
$$

These values for $\lambda=0$ ensure that our one-loop results (21) and (22) for $Z_{1}$ and $Z_{c}$ reduce to their $m=0$ analogs ${ }^{33}$ when $\lambda$ is set to zero.

\section{RG equations}

Upon changing $\mu$ at fixed values of the bare interaction constants, we see that the renormalized functions $G_{\text {ren }}^{(N, M)}=Z_{\phi}^{-(N+M) / 2} Z_{1}^{-M / 2} G^{(N, M)}$ satisfy the $\mathrm{RG}$ equations

$$
\left[\mathcal{D}_{\mu}+\frac{N+M}{2} \eta_{\phi}+\frac{M}{2} \eta_{1}\right] G_{\mathrm{ren}}^{(N, M)}=0
$$

with

$$
\mathcal{D}_{\mu} \equiv \mu \partial_{\mu}+\sum_{\wp=u, \sigma, \tau, \rho, c, \lambda} \beta_{\wp} \partial_{\wp} .
$$

Here $\eta_{\phi}(u)$ and $\eta_{1}(u, \lambda)$ denote particular ones of the functions

$$
\left.\eta_{\wp} \equiv \mu \partial_{\mu}\right|_{0} \ln Z_{\wp}, \quad \wp=\phi, u, \sigma, \tau, \rho, 1, c,
$$

where $\left.\mu \partial_{\mu}\right|_{0}$ stands for a $\mu$ derivative at fixed bare interaction constants $\stackrel{\circ}{\dot{u}}, \stackrel{\circ}{\tau}, \stackrel{\circ}{\rho}, \stackrel{\circ}{\sigma}, \stackrel{\circ}{c}$, and $\stackrel{\circ}{\lambda}$.

The beta functions appearing in Eq. (28) are defined via

$$
\left.\beta_{\wp} \equiv \mu \partial_{\mu}\right|_{0} \wp, \quad \wp=u, \sigma, \tau, \rho, c, \lambda .
$$

They can be conveniently expressed in terms of the $\eta_{\wp}$,

$$
b_{\tau}(u) \equiv A_{\tau}\left[\left.\mu \partial_{\mu}\right|_{0} \ln A_{\tau}+\eta_{\tau}-2 \eta_{\rho}\right]
$$

and

$$
b_{c}(u, \lambda)=A_{c}\left[\left.\mu \partial_{\mu}\right|_{0} \ln A_{c}+\eta_{c}-\eta_{\rho}\right] .
$$

We have

$$
\begin{aligned}
\beta_{u}(u, \epsilon) & =-u\left[\epsilon+\eta_{u}(u)\right], \\
\beta_{\sigma}(u, \sigma) & =-\sigma \eta_{\sigma}(u), \\
\beta_{\tau}(u, \tau, \rho) & =-\tau\left[2+\eta_{\tau}(u)\right]-\rho^{2} b_{\tau}(u), \\
\beta_{\rho}(u, \rho) & =-\rho\left[1+\eta_{\rho}(u)\right], \\
\beta_{c}(u, \lambda, \rho, c) & =-c\left[1+\eta_{c}(u, \lambda)\right]-\rho b_{c}(u, \lambda),
\end{aligned}
$$

and

$$
\beta_{\lambda}(u, \lambda)=\frac{-\beta_{u}(u, \epsilon) \partial_{u} P_{\lambda}(u, \lambda, \epsilon)}{1+\partial_{\lambda} P_{\lambda}(u, \lambda, \epsilon)} .
$$

That the functions $\eta_{u, \sigma, \tau, \rho}(u), b_{\tau}(u), \eta_{c, 1}(u, \lambda), b_{c}(u, \lambda)$, and $\beta_{\lambda}(u, \lambda)$ are independent of $\epsilon$ is due to our use of the minimal subtraction prescription. As usual, the eta functions can be written in terms of $u$ derivatives of the residues of the $Z$ factors; we have

$$
\eta_{\wp}(u, \lambda)=-u \partial_{u} \operatorname{Res}_{\epsilon=0} Z_{\wp}(u, \lambda, \epsilon), \wp=\phi, u, \sigma, \tau, \rho, 1, c .
$$

The analogous results for $b_{\tau}, b_{c}$, and $\beta_{\lambda}$,

$$
\begin{gathered}
b_{\tau}(u)=-u \partial_{u} \operatorname{Res}_{\epsilon=0} A_{\tau}(u, \epsilon), \\
b_{c}(u, \lambda)=-u \partial_{u} \operatorname{Res}_{\epsilon=0} A_{c}(u, \lambda, \epsilon),
\end{gathered}
$$

and

$$
\begin{aligned}
\beta_{\lambda}(u, \lambda) & =u \partial_{u} \operatorname{Res}_{\epsilon=0} P_{\lambda}(u, \lambda, \epsilon) \\
& =\sum_{i=1}^{\infty} i P_{\lambda}^{(i,-1)}(\lambda) u^{i}
\end{aligned}
$$

can be derived from Eqs. (31), (32), and (34), respectively.

Upon substituting Eq. (17) into Eq. (36), we obtain

$$
b_{\tau}(u)=\frac{n+2}{3} \frac{m u}{16}+O\left(u^{2}\right) \text {. }
$$

Our perturbative result for $\beta_{\lambda}$ will be given and discussed below [see Eqs. (55) and (59)-(61), and Fig. 1]. The function $b_{c}(u)$ will not be computed in this paper since its explicit form is not needed for our subsequent analysis.

\section{Flow equations and fixed points}

To exploit the RG equations (27) via characteristics, we introduce running coupling constants $\bar{\varnothing}(\ell)$ into which the $\wp$ evolve under a change $\mu \rightarrow \bar{\mu}(\ell)=\mu \ell$ of the momentum scale. They are solutions to the flow equations

$$
\ell \frac{d}{d \ell} \bar{\wp}(\ell)=\beta_{\wp}[\bar{u}(\ell), \ldots], \quad \wp=u, \sigma, \tau, \rho, c, \lambda
$$


satisfying the initial conditions

$$
\bar{\wp}(1)=\wp, \quad \wp=u, \sigma, \tau, \rho, c, \lambda .
$$

As is well known, for dimensions $d<d^{*}(m)$ the bulk critical behavior at the Lifshitz point $\tau=\rho=0$ (with $\sigma>0$ ) is described by a fixed point that is located at the nontrivial zero $u^{*}=u^{*}(m, \epsilon)$ of the beta function $\beta_{u}$. According to Eq. (60) of Ref. 15, the expansion to order $\epsilon^{2}$ of this root is given by

$$
\begin{aligned}
u^{*}= & \frac{2 \epsilon}{3} \frac{9}{n+8}+\frac{8 \epsilon^{2}}{27}\left[\frac{9}{n+8}\right]^{3}\left\{3 \frac{5 n+22}{27} J_{u}(m)\right. \\
& \left.+\frac{1}{24} \frac{n+2}{3}\left[\frac{j_{\sigma}(m)}{8(m+2)}-j_{\phi}(m)\right]\right\}+O\left(\epsilon^{3}\right),
\end{aligned}
$$

where $J_{u}(m)$ is one of the four single integrals $j_{\phi}(m)$, $j_{\sigma}(m), j_{\rho}(m)$, and $J_{u}(m)$ in terms of which the two-loop results for the bulk renormalization factors $Z_{\phi}(u), Z_{\sigma}(u)$, $Z_{\rho}(u), Z_{\tau}(u)$, and $Z_{u}(u)$ were expressed in Ref. 15 . It is given by

$$
J_{u}(m)=1-\frac{C_{E}+\psi\left(2-\frac{m}{4}\right)}{2}+j_{u}(m),
$$

where $C_{E}=0.577216 \ldots$ denotes Euler's constant while $\psi(x)$ is the digamma function and $j_{u}(m)$ means the integral

$$
j_{u}(m)=\frac{B_{m}}{2^{4+m} \pi^{(6+m) / 4}} \int_{0}^{\infty} d v v^{m-1} \Phi_{m, d^{*}}^{2}(v) \Theta_{m}(v)
$$

with

$$
\begin{aligned}
\Theta_{m}(v)= & \frac{v^{4}}{32} \frac{1}{\Gamma\left(\frac{3}{2}+\frac{m}{4}\right)}{ }_{2} F_{3}\left(1,1 ; \frac{3}{2}, 2, \frac{3}{2}+\frac{m}{4} ; \frac{v^{4}}{64}\right) \\
& -\frac{v^{2}}{4} \frac{\sqrt{\pi}}{\Gamma\left(1+\frac{m}{4}\right)}{ }_{1} F_{2}\left(\frac{1}{2} ; \frac{3}{2}, 1+\frac{m}{4} ; \frac{v^{4}}{64}\right)
\end{aligned}
$$

and

$$
B_{m} \equiv \frac{S_{4-\frac{m}{2}} S_{m}}{F_{m, 0}^{2}}=\frac{2^{10+m} \pi^{6+\frac{3 m}{4}} \Gamma\left(\frac{m}{2}\right)}{\Gamma\left(2-\frac{m}{4}\right) \Gamma\left(\frac{m}{4}\right)^{2}} .
$$

The quantity $S_{m}$ denotes the surface area $S_{m} \equiv$ $2 \pi^{m / 2} / \Gamma(m / 2)$ of an $m$-dimensional unit sphere.

Since some of our analytical results to be given below involve besides the functions $j_{\phi}(m)$ and $j_{\sigma}(m)$ also $j_{\rho}(m)$, let us recall their definition here for completeness. We have

$$
j_{\phi}(m) \equiv B_{m} J_{0,3}(m)
$$

and

$$
j_{\sigma}(m) \equiv B_{m} J_{4,3}(m)
$$

where $J_{0,3}(m)$ and $J_{4,3}(m)$ are special cases of the integral

$$
J_{p, s}(m) \equiv \int_{0}^{\infty} v^{m-1+p} \Phi_{m, d^{*}}^{s}(v) d v,
$$

previously considered (even for $d \neq d^{*}$ ) in Ref. 14. Further,

$$
j_{\rho}(m) \equiv B_{m} \int_{0}^{\infty} d v v^{m+1} \Phi_{m, d^{*}}^{2}(v) \Xi_{m, d^{*}}(v),
$$

with

$$
\Xi_{m, d^{*}}(v)=\frac{v^{(4-m) / 2}}{32(2 \pi)^{\frac{4+m}{4}}}\left[I_{\frac{m-4}{4}}\left(\frac{v^{2}}{4}\right)-\mathbf{L}_{\frac{m-4}{4}}\left(\frac{v^{2}}{4}\right)\right],
$$

where $I_{\nu}$ and $\mathbf{L}_{\nu}$ denote modified Bessel and Struve functions, respectively. ${ }^{34}$

Here we are interested in the surface critical behavior at the analog of the ordinary transition that occurs at the Lifshitz point (LP). Whenever it is necessary to distinguish this type of ordinary transition from its counterpart taking place on the ferromagnetic section of the critical line (CL), we shall refer to the former as LP ordinary transition and to the latter as CL ordinary transition. The latter belongs, of course, to the surface universality class of the usual ordinary transition at a critical point $(\mathrm{CP})$, which is described by the standard semi-infinite $n$-vector model. ${ }^{28}$

Let us note some characteristic features of the LP ordinary transition, which serve to identify it and distinguish it from other types of surface transitions such as the special and extraordinary ones:

(i) The surface is disordered on the bulk disordered side of the transition. Hence the order-parameter profile $\langle\phi(\boldsymbol{x})\rangle$, and especially the local surface order parameter $\left\langle\phi^{\mathfrak{B}}(\boldsymbol{r})\right\rangle=\langle\phi(\boldsymbol{r}, z=0)\rangle$, vanish at the transition.

(ii) The surface does not become critical on its own but remains noncritical at the transition. That is, the ir singularities which occur at the surface are induced by the bulk, and the surface susceptibility

$$
\chi_{11}(\boldsymbol{p})=\hat{G}^{(0,2)}\left(\boldsymbol{p} ; z=0, z^{\prime}=0\right)
$$

diverges neither at $\boldsymbol{p}=\mathbf{0}$ nor at any nonzero value of the momentum $\boldsymbol{p}$, so that its inverse is strictly positive:

$$
\chi_{11}^{-1}(\boldsymbol{p})>0, \quad \forall \boldsymbol{p} .
$$

In the case of the LP special transition, condition (i) continues to apply. However, Eq. (53) holds only for nonzero momenta $\boldsymbol{p}$ because the surface becomes critical as well; i.e., the surface susceptibility $\chi_{11} \equiv \chi_{11}(\mathbf{0})$ diverges, $\chi_{11}^{-1}(\mathbf{0})=0$. On the other hand, at the LP extraordinary transition, $m_{1} \equiv\left\langle\phi^{\mathfrak{B}}\right\rangle$ does not vanish. Therefore (i) ceases to hold while (ii) still applies for all $\boldsymbol{p}$ (barring eventual Goldstone singularities at $\boldsymbol{p}=\mathbf{0}$ due 


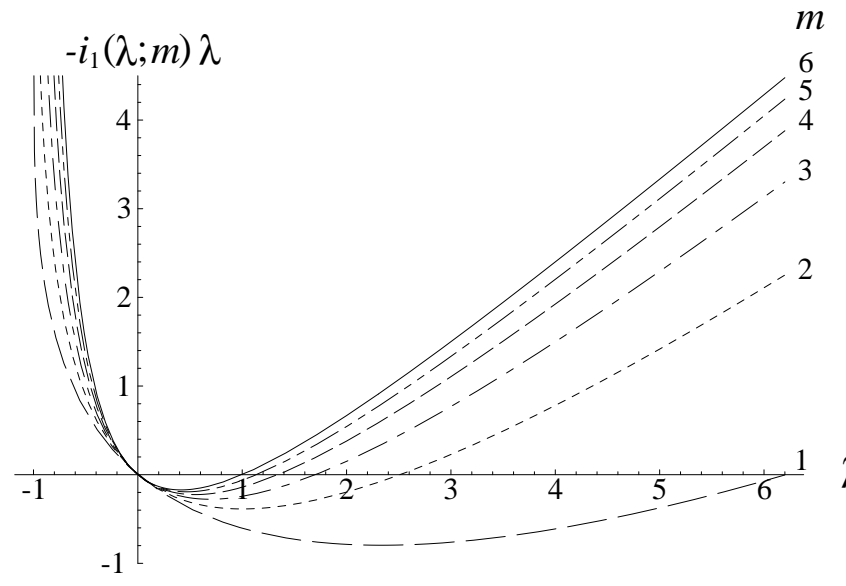

FIG. 1: The functions $-i_{1}(\lambda ; m) \lambda$ for $m=1,2, \ldots, 6$.

to the spontaneous breaking of the continuous $O(n)$ symmetry for $n \geq 2$ in sufficiently high dimensions). ${ }^{35}$

From the form of the beta function $\beta_{c}$ in Eq. (33) for $\rho=0$ we can read off the fixed-point values $c^{*}= \pm \infty$ and $c^{*}=0$ of $c$; all others would require that

$$
\eta_{c}\left(u^{*}, \lambda^{*}\right)=-1
$$

We ignore this possibility since we see no reason why the zeros $\lambda=\lambda^{*}$ of the function $\beta_{\lambda}\left(u^{*}, \lambda\right)$ should satisfy Eq. (54). (By inspection of the perturbation series for $\eta_{c}$ and $\beta_{\lambda}$ to low orders in $u$ one can convince oneself that this condition cannot hold as an identity in $\epsilon$.)

Recalling that the line of $\mathrm{CP}$ ordinary transitions is mapped onto a fixed point with $c=c_{\text {ord }}^{*} \equiv \infty$, we expect the fixed point describing the critical behavior at the LP ordinary transition to be located at $c=\infty$ as well. In the special case $m=0$, in which the variable $\dot{\lambda}$ drops out, this is evident because the results must reduce to those for the usual isotropic semi-infinite $\phi^{4}$ model (cf. Refs. 28 and 36-39). However, for $m>0$ this remains to be verified.

To this end we need some information about the flow in the $c \lambda$ hyperplane at $u=u^{*}$ and $\rho=\tau=0$. Upon inserting the $O(u)$ result (23) for $P_{\lambda}$ into Eq. (38), we arrive at a two-loop expression of the form

$\beta_{\lambda}(u, \lambda)=-\frac{n+2}{6} i_{1}(\lambda ; m) \lambda u+2 u^{2} P^{(2,-1)}(\lambda)+O\left(u^{3}\right)$

Let us first consider $\beta_{\lambda}\left(u^{*}, \lambda\right)$ in the one-loop approximation, i.e., to first order in $u$. Its fixed-point values are given by the zeros of the functions $-\lambda i_{1}(\lambda ; m)$, which are plotted for $m=1,2 \ldots, 6$ in Fig. 1 .

From their form we see that the trivial zeros $\lambda=0$ are ir unstable in the $\lambda$ direction. There also exist nontrivial positive roots $\lambda_{+}^{*}$ for $1 \leq m \leq 6$, given by the zeros $\lambda_{0}(m)$ of the functions $i_{1}(\lambda ; m)$ :

$$
i_{1}\left(\lambda_{0} ; m\right)=0 \text { for } \lambda_{0}(m)= \begin{cases}6.2092 & \text { for } m=1 \\ 2.5129 & \text { for } m=2 \\ 1.7018 & \text { for } m=3 \\ 1.3425 & \text { for } m=4 \\ 1.1363 & \text { for } m=5 \\ 1 & \text { for } m=6 .\end{cases}
$$

Since $\beta_{\lambda}\left(u^{*}, \lambda\right)$ has positive slopes at $\lambda_{0}(m)$, these fixed points are ir stable in the $\lambda$ direction.

At two-loop order the trivial zeros get shifted to

$$
\lambda^{*}=\frac{72 P^{(2,-1)}(0)}{(n+2)(n+8)} \epsilon+O\left(\epsilon^{2}\right) .
$$

In deriving this result we have substituted $u^{*}$ by it $\epsilon$ expansion (42).

The associated RG eigenvalues (which govern the behavior $\bar{\lambda}(\ell)-\lambda^{*} \sim \ell^{-y_{\lambda}}$ of the running variable $\bar{\lambda}$ near $\left.\lambda_{*}\right)$ are

$$
y_{\lambda} \equiv-\left(\partial_{\lambda} \beta_{\lambda}\right)\left(u^{*}, \lambda^{*}\right)=\frac{n+2}{n+8} \epsilon+O\left(\epsilon^{2}\right) .
$$

According to this $O(\epsilon)$ result, $y_{\lambda}>0$ for $\epsilon>0$. Thus the fixed points with $\lambda=\lambda^{*}$ are indeed ir unstable.

The two-loop function $P^{(2,-1)}(\lambda)$ is as yet unknown, but in Appendix D we calculate its value at $\lambda=0$, showing that it is negative. In the special cases $m=2$ and $m=6$, it can be computed analytically. One obtains

$$
\begin{array}{r}
\left.P^{(2,-1)}(0)\right|_{m=2}=-\frac{n+2}{3} \frac{1}{192}\left[5 \pi^{2}-16 \ln 2\right. \\
\left.-6 \ln ^{2} 3-12 \operatorname{Li}_{2}(1 / 3)\right]
\end{array}
$$

and

$$
\left.P^{(2,-1)}(0)\right|_{m=6}=-\frac{n+2}{3} \frac{1}{6}
$$

where $\operatorname{Li}_{2}(x)=\sum_{k=1}^{\infty} x^{k} / k^{2}$ is the dilogarithm, giving $\mathrm{Li}_{2}(1 / 3)=0.366213 \ldots$. For other choices of $m$, the integrals in terms of which $P^{(2,-1)}(0)$ is expressed in Appendix D [see Eqs. (B5), (B6), and (D7)] can be determined by numerical means (see Appendix E). Our results

$$
P^{(2,-1)}(0)=-\frac{n+2}{3}\left\{\begin{array}{l}
0.12473 \text { for } m=1 \\
0.13865 \text { for } m=2, \\
0.14885 \text { for } m=3 \\
0.15652 \text { for } m=4 \\
0.16231 \text { for } m=5 \\
0.16667 \text { for } m=6
\end{array}\right.
$$

demonstrate that $P^{(2,-1)}(0)$ does not vanish.

In order to understand the meaning of the fixed point at $(u, c, \lambda)=\left(u^{*}, 0, \lambda^{*}\right)$, one should note that the derivative $\partial \chi_{11}^{-1}(\boldsymbol{p}) /\left.\partial\left(p_{\alpha} p_{\alpha}\right)\right|_{\boldsymbol{p}=\mathbf{0}}$ of the zero-loop inverse surface susceptibility vanishes at $\lambda=0$. This behavior of $\chi_{11}^{-1}(\boldsymbol{p})$ is reminiscent of the vanishing of the derivative 
$\partial \tilde{\Gamma}_{\mathrm{b}}^{(2)}(\boldsymbol{q}, \tau=0, \rho) /\left.\partial\left(q_{\alpha} q_{\alpha}\right)\right|_{\boldsymbol{q}=\mathbf{0}}$ of its bulk analog, the bulk vertex function $\tilde{\Gamma}_{\mathrm{b}}^{(2)}(\boldsymbol{q})=\int d^{d} x \Gamma_{\mathrm{b}}^{(2)}(\boldsymbol{x}) e^{i \boldsymbol{q} \cdot \boldsymbol{x}}$, at $\rho=0$. Clearly, if $\lambda$ becomes negative, then stabilizing surface contributions to the Hamiltonian of fourth order in $\partial_{\alpha}$ and second order in $\phi$ will be needed. In sufficiently high space dimensions $d$, a phase with modulated surface order and a surface Lifshitz point should exist for such a generalized model, at least when the bulk parameters $\tau$, $\rho$, and $\sigma$ are in a regime for which the bulk is disordered. Beyond Landau theory, fluctuations are expected to shift the location of the "instability point" of the surface susceptibility (i.e., where $\partial \chi_{11}^{-1}(\boldsymbol{p}) /\left.\partial\left(p_{\alpha} p_{\alpha}\right)\right|_{\boldsymbol{p}=0}$ vanishes if $c=\rho=\tau=0)$ to a nonzero value of $\lambda$. Hence it is reasonable that the fixed point associated with this surface wave-vector instability gets shifted to the nontrivial value (57) of $\lambda$.

In $d=3$ (bulk) dimensions, no surface Lifshitz point is expected to occur at absolute temperatures $T>0$ for models of the kind considered (with short-range interactions) because the bulk phase in $d-1$ dimensions to which the required surface phase with long-range modulated order would correspond to is believed to be thermodynamically unstable. ${ }^{3,40}$ Thus the fixed point at $(u, \lambda)=\left(u^{*}, \lambda^{*}\right)$ and $c=\rho=\tau=0$ is of little interest. Since our main concern here is the ordinary LP transition, we will refrain in the sequel from a detailed investigation of the multicritical behavior it may describe in dimensions $d>3$.

Next, we consider the nontrivial zeros $\lambda_{+}^{*}$ of $\beta_{\lambda}\left(\lambda, u^{*}\right)$. Using the two-loop expression (55) in conjunction with the previously utilized result (42) for $u^{*}$, one obtains

$$
\lambda_{+}^{*}(m)=\lambda_{0}+\frac{72 P_{\lambda}^{(2,-1)}\left(\lambda_{0}\right) \epsilon}{(n+2)(n+8) \lambda_{0} i_{1}^{\prime}\left(\lambda_{0} ; m\right)}+O\left(\epsilon^{2}\right),
$$

where $i_{1}^{\prime}(\lambda ; m) \equiv \partial i_{1}(\lambda ; m) / \partial \lambda$.

For the associated correction-to-scaling exponent

$$
\omega_{\lambda} \equiv\left(\partial_{\lambda} \beta_{\lambda}\right)\left(u^{*}, \lambda_{+}^{*}\right),
$$

which governs the behavior $\bar{\lambda}-\lambda_{+}^{*} \sim \ell^{\omega_{\lambda}}$ of the running variable $\bar{\lambda}$ near $\lambda_{+}^{*}$, we find the result

$$
\omega_{\lambda}=-\frac{n+2}{n+8} i_{1}^{\prime}\left(\lambda_{0} ; m\right) \lambda_{0} \epsilon+O\left(\epsilon^{2}\right) .
$$

By performing the differentiation $\partial / \partial \lambda$ inside of the integral (24), or from Fig. 1, one can easily see that $i_{1}^{\prime}(\lambda ; m)<0$ for $\lambda>0$. Hence $\omega_{\lambda}>0$ to linear order in $\epsilon>0$.

A schematic picture of the flow is depicted in Fig. 2. On the line $\lambda=\lambda_{+}^{*}$ in the hyperplane $u=u^{*}$ we can identify the three fixed points

$$
\begin{array}{ll}
\mathcal{P}_{\text {ord }}^{*}: & \left(c_{\text {ord }}^{*}=\infty, \quad \lambda=\lambda_{+}^{*}\right), \\
\mathcal{P}_{\mathrm{sp}}^{*}: & \left(c_{\mathrm{sp}}^{*}=0, \quad \lambda=\lambda_{+}^{*}\right), \\
\mathcal{P}_{\mathrm{ex}}^{*}: & \left(c_{\mathrm{ex}}^{*}=-\infty, \quad \lambda=\lambda_{+}^{*}\right) .
\end{array}
$$

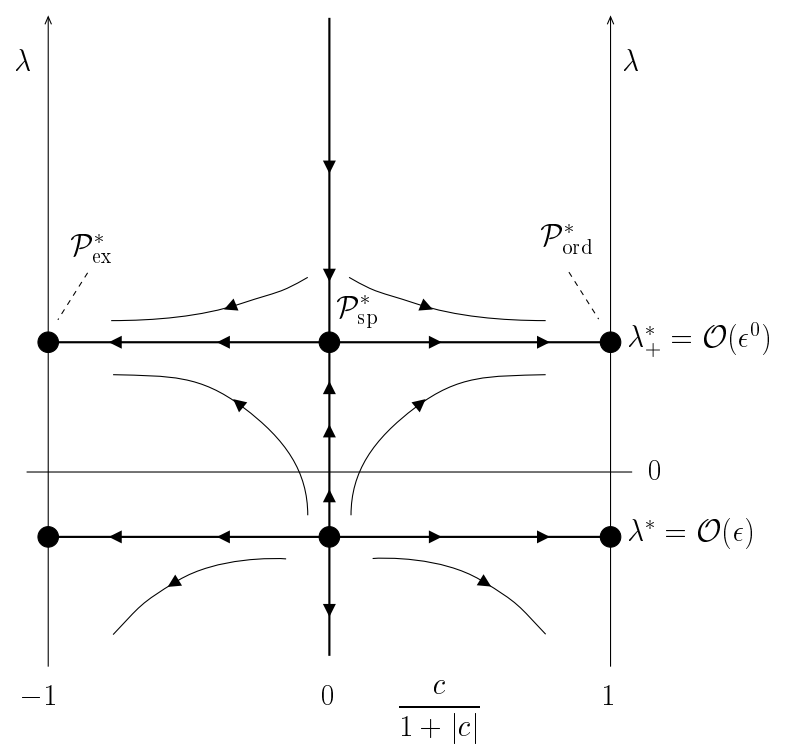

FIG. 2: Schematic picture of the RG flow in the $c \lambda$ plane at $\rho=\tau=0$ and $u=u^{*}$, showing the fixed points $\mathcal{P}_{\text {ord }}^{*}, \mathcal{P}_{\mathrm{sp}}^{*}$, and $\mathcal{P}_{\text {ex }}^{*}$ specified in Eq. (65).

All three are ir stable along the $\lambda$ direction. As the notation suggests, we therefore expect them to describe the LP ordinary, special, and extraordinary transitions, respectively.

\section{RG ANALYSIS OF THE LP ORDINARY TRANSITION}

\section{A. Reduction to cumulants with $\stackrel{\circ}{c}=\infty$ and $\stackrel{\AA}{\lambda}=0$}

Assuming that the initial values $u, c$, and $\lambda$ belong to the basin of attraction of the fixed point $\mathcal{P}_{\text {ord }}^{*}$, we now specialize to the case of the LP ordinary transition. From the explicit form (7) of the free propagator $G$ and the boundary condition (6) it satisfies we see that in the limit $\stackrel{\circ}{\rightarrow} \rightarrow \infty$ at fixed $\grave{\lambda}>0$, the dependence on $\grave{\lambda}$ drops out and $G$ turns into the Dirichlet propagator

$$
G_{\mathrm{D}}\left(\boldsymbol{x}, \boldsymbol{x}^{\prime}\right)=G_{\mathrm{b}}\left(\boldsymbol{x}-\boldsymbol{x}^{\prime}\right)-G_{\mathrm{b}}\left(\boldsymbol{x}-\boldsymbol{x}^{\prime}+2 z^{\prime} \boldsymbol{n}\right) .
$$

Both properties, the Dirichlet boundary condition as well as the $\dot{\lambda}$ independence for $\stackrel{\imath}{c} \rightarrow \infty$, carry over to each individual Feynman integral of the bare (dimensionally regularized) correlation functions $G^{(N, M)}$. Thus they hold at least to any finite order of perturbation theory.

Now, suppose we compute the renormalized functions $G_{\text {ren }}^{(N, M)}$ using RG-improved perturbation theory. The RG maps these functions with given initial values $u, c$, and $\lambda$ to their RG images at points on the RG trajectory with the values $\bar{u}(\ell), \bar{c}(\ell)$, and $\bar{\lambda}(\ell)$. To obtain the asymptotic behavior of the $G_{\mathrm{ren}}^{(N, M)}$ in the large lengthscale limit $\ell \rightarrow 0$, we may replace the running interac- 
tions constants $\bar{u}, \bar{c}$, and $\bar{\lambda}$ by their limiting values $u^{*}$, $c_{\text {ord }}^{*}=\infty$, and $\lambda_{+}^{*}$ for $\ell \rightarrow 0$ provided the RG images of the correlation functions remain finite and nonzero in this limit. Below the upper critical dimension $d^{*}(m)$ (i.e., for $\epsilon>0$ ), there is no reason to expect any problems in the limits $\bar{u} \rightarrow u^{*}$ and $\bar{\lambda} \rightarrow \lambda_{+}^{*}$ : According to RG-improved perturbation theory, neither $\lambda-\lambda_{+}^{*}$ nor $u-u^{*}$ should be dangerous irrelevant variables. ${ }^{41,42}$

On the other hand, as $\bar{c} \rightarrow \infty$, all functions $G_{\mathrm{ren}}^{(N, M)}$ with $M>0$ should vanish. To see this, note that the boundary condition (4) yields

$$
\hat{\phi}_{p}^{\mathfrak{B}}=\stackrel{\circ}{c_{p}^{-1}} \partial_{n} \hat{\phi}_{\boldsymbol{p}}
$$

for the Fourier transform of the bare boundary operator $\phi^{\mathfrak{B}}$, where $\stackrel{\circ}{c}_{\boldsymbol{p}}$ was defined in Eq. (8). Using this and expanding in powers of $1 /{ }^{\circ}$, one sees that the leading contribution of the bare cumulants $G^{(N, M)}$ in the limit $\stackrel{\circ}{c} \rightarrow \infty$ (with all other interaction constants kept fixed), is given by

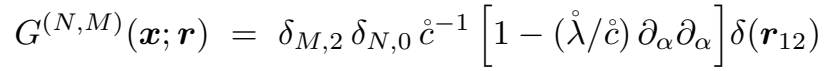

$$
\begin{aligned}
& +\stackrel{\circ}{c}-M G_{\infty}^{(N, M)}(\boldsymbol{x} ; \boldsymbol{r}) \text {, }
\end{aligned}
$$

where $\boldsymbol{r}_{12}$ denotes the displacement $\boldsymbol{r}_{1}-\boldsymbol{r}_{2}$ of the two surface points in the case $(N, M)=(0,2)$. The functions on the right-hand side are defined through

$$
G_{\infty}^{(N, M)}(\boldsymbol{x} ; \boldsymbol{r})=\left\langle\prod_{j=1}^{N} \phi_{a_{j}}\left(\boldsymbol{x}_{j}\right) \prod_{k=1}^{M} \partial_{n} \phi_{b_{k}}\left(\boldsymbol{r}_{k}\right)\right\rangle_{c=\infty, \grave{\lambda}=0}^{\mathrm{cum}} .
$$

The extra term for $(N, M)=(0,2)$ in Eq. (68) is produced by the zero-loop contribution to $G^{(0,2)}$. Equation (68) generalizes the $m=0$ result (3.133) of Ref. 28 to the $m>0$ case.

The large- $\stackrel{c}{c}$ behavior (68) of the bare functions $G^{(N, M)}$ implies that the RG images of their renormalized counterparts $G_{\mathrm{ren}}^{(N, M)}$ vary $\sim \bar{c}^{M(-1+O(\epsilon))}$ as $\bar{c} \rightarrow \infty$, aside from extra terms proportional to $\delta\left(\boldsymbol{r}_{12}\right)$ and $\partial_{\alpha} \partial_{\alpha} \delta\left(\boldsymbol{r}_{12}\right)$ for $(N, M)=(0,2)$. All in all, the following conclusions can be drawn:

(i) The $\lambda$ dependence that remains when corrections to scaling $\sim \lambda-\lambda_{+}^{*}$ are ignored (upon making the replacement $\bar{\lambda} \rightarrow \lambda_{+}^{*}$ ) is restricted to nonuniversal amplitudes (which are expressible via appropriate RG trajectory integrals $\left.{ }^{43}\right)$.

(ii) The renormalized cumulants $G_{\mathrm{ren}}^{(N, M)}$ approach zero in the limit $\bar{c} \rightarrow \infty$ whenever $M>0$, and satisfy Dirichlet boundary conditions. In other words, taken at $\bar{c} \rightarrow \infty$, they vanish if one or several positions $\boldsymbol{x}_{j}$ of the operators $\phi_{\text {ren }}\left(\boldsymbol{x}_{j}\right)$ approach the surface. ${ }^{44}$

(iii) The critical behavior of the $G^{(N, M)}$ at the LP ordinary transition must match that of the $G_{\infty}^{(N, M)}$, and hence should be derivable in much the same way as in the $m=0$ case by a direct RG analysis of the latter functions.

\section{B. Renormalization of the cumulants $G_{\infty}^{(N, M)}$}

To set up such an analysis, let us consider the renormalization of the cumulants $G_{\infty}^{(N, M)}$. Adding source terms to the Hamiltonian, we introduce the action

$$
\begin{aligned}
\mathcal{A}\left[\boldsymbol{\phi} ; \boldsymbol{J}, \boldsymbol{J}_{1, \infty}\right]= & \int_{\mathfrak{V}} d V \boldsymbol{J} \cdot \boldsymbol{\phi}+\int_{\mathfrak{B}} d A \boldsymbol{J}_{1, \infty} \cdot \partial_{n} \phi \\
& -\left.\mathcal{H}[\boldsymbol{\phi}]\right|_{\hat{c}=\infty, \grave{\lambda}=0},
\end{aligned}
$$

in which the Hamiltonian is given by Eqs. (1)-(3) with

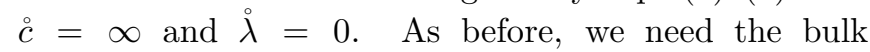
reparametrizations (16). The surface operator $\partial_{n} \phi$ is multiplicatively renormalizable; we introduce its renormalized counterpart $\left(\partial_{n} \phi\right)_{\text {ren }}$ via

$$
\partial_{n} \phi=\left[Z_{\phi}(u, \epsilon) Z_{1, \infty}(u, \epsilon)\right]^{1 / 2}\left(\partial_{n} \phi\right)_{\text {ren }} .
$$

Since the Fourier transform $\hat{G}^{(0,2)}(\boldsymbol{p})$ has $\mu$-dimension one, it may contain additional primitive uv divergences localized on the surface of the form $\mu\left(C_{0}+\rho C_{1}\right)+$ $\sigma^{1 / 2} p_{\alpha} p_{\alpha} C_{2}$, where the renormalization functions $C_{0}$, $C_{1}$ and $C_{2}$ would diverge as $C_{0} \sim \Lambda, C_{1} \sim \ln \Lambda$, and $C_{2} \sim \ln \Lambda$ if we regularized the theory's uv singularities by a large-momentum cutoff $\Lambda$. Thus the generating functional of the renormalized functions $G_{\infty, \text { ren }}^{(N, M)}$ can be written as

$$
\begin{gathered}
\mathcal{G}_{\infty, \text { ren }}\left[\boldsymbol{J}, \boldsymbol{J}_{1, \infty}\right] \\
=\ln \operatorname{Tr}_{\boldsymbol{\phi}} \exp \left\{\mathcal{A}\left[\boldsymbol{\phi} ; \boldsymbol{J} / \sqrt{Z_{\phi}}, \boldsymbol{J}_{1, \infty} / \sqrt{Z_{\phi} Z_{1, \infty}}\right]\right. \\
+\int_{\mathfrak{B}} d A\left[\mu\left(C_{0}+\rho C_{1}\right) \boldsymbol{J}_{1, \infty}^{2}\right. \\
\left.\left.\quad+\frac{1}{2} C_{2} \sigma^{1 / 2} \sum_{\alpha=1}^{m}\left(\partial_{\alpha} \boldsymbol{J}_{1, \infty}\right)^{2}\right]\right\},
\end{gathered}
$$

where $\operatorname{Tr}_{\phi}$ means functional integration over $\phi$. Hence we have

$$
\begin{aligned}
G_{\infty, \text { ren }}^{(N, M)}= & {\left[\mu\left(C_{0}+\rho C_{1}\right)-\sigma^{1 / 2} C_{2} \partial_{\alpha} \partial_{\alpha}\right] \delta_{M, 2}^{N, 0} \delta\left(\boldsymbol{r}_{12}\right) } \\
& +Z_{\phi}^{-(N+M) / 2} Z_{1, \infty}^{-M / 2} G_{\infty}^{(N, M)}
\end{aligned}
$$

for the renormalized cumulants in position space.

In our dimensional regularization scheme, $C_{0}$ is finite and hence can be chosen to vanish. The $\ln \Lambda$ singularities of $C_{1}$ and $C_{2}$ translate into poles at $\epsilon=0$. For convenience, we fix $Z_{1, \infty}$ and $C_{2}$ by requiring that the poles of $\left.\hat{G}^{(1,1)}(\boldsymbol{p}, z)\right|_{\rho=0}$ and the additional ones of $\left.\hat{G}^{(0,2)}(\boldsymbol{p})\right|_{\rho=0}$ be minimally subtracted, respectively. Likewise, $C_{1}$ could be determined by requiring that the additional poles of $\left[\partial_{\rho} \hat{G}^{(0,2)}(\boldsymbol{p})\right]_{\rho=0}$ be minimally subtracted. However, the explicit two-loop expression for $C_{1}$ will not be needed in the sequel, so we refrain from determining it.

The graphs of $\hat{G}_{\infty}^{(1,1)}(\boldsymbol{p}, z)$ to two-loop order are shown in Fig. 3. To this order, the only graph contributing to

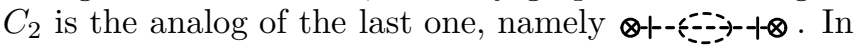




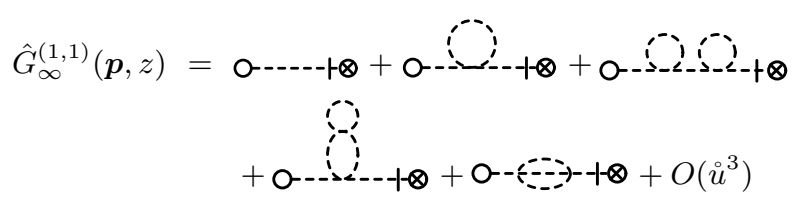

FIG. 3: Graphs of $\hat{G}_{\infty}^{(1,1)}(\boldsymbol{p}, z)$ to two-loop order. The dashed line denotes the free Dirichlet propagator $\hat{G}_{D}$. The vertical bars signify normal derivatives $\partial_{n}$. Open and crossed circles indicate external points off and on the surface, respectively.

Appendixes A and B the Laurent expansions of these graphs for $\rho=\tau=0$ are computed to the required order in $\epsilon$. Using these one arrives at the results

$$
\begin{aligned}
Z_{1, \infty}=1 & +\frac{n+2}{3} \frac{u}{2 \epsilon}+\frac{(n+2)(n+5)}{36} \frac{u^{2}}{\epsilon^{2}} \\
& +\frac{n+2}{3}\left[j_{1}(m)-J_{u}(m)\right] \frac{u^{2}}{4 \epsilon}+O\left(u^{3}\right)
\end{aligned}
$$

and

$$
\begin{aligned}
C_{2}(u, \epsilon)= & -\frac{u^{2}}{\epsilon} \frac{n+2}{3} b_{m}\left[\frac{2}{3} \frac{J_{2,3}(m)}{6-m}\right. \\
& \left.-I^{(1)}(m)+I^{(2)}(m)\right]+O\left(u^{3}\right) .
\end{aligned}
$$

Here $b_{m}$ is the constant introduced by Eq. (B3), $I^{(1)}(m)$ and $I^{(2)}(m)$ are the integrals defined by Eqs. (B5) and (B6), respectively, while $J_{2,3}(m)$ denotes a particular one of the integrals (49).

The quantity

$$
j_{1}(m) \equiv B_{m} \int_{0}^{\infty} d y y^{m-5} \Phi_{m, d^{*}}(y) \int_{0}^{y} d v v^{3} \Phi_{m, d^{*}}^{2}(v)
$$

is a new integral, not encountered in the bulk case. It is not difficult to see that it can be reduced to a single integral. Rewriting the double integral (76) as $\int_{0}^{\infty} d v \int_{v}^{\infty} d y \ldots$ gives

$$
j_{1}(m)=B_{m} \int_{0}^{\infty} d v v^{3} \Phi_{m, d^{*}}^{2}(v) \Omega_{m, d^{*}}(v)
$$

with

$$
\Omega_{m, d}(v) \equiv \int_{v}^{\infty} d y y^{m-5} \Phi_{m, d}(y) .
$$

The latter integration can be performed analytically; at $d=d^{*}(m)$ the result becomes

$$
\begin{aligned}
\Omega_{m, d^{*}}(v)= & \frac{\sqrt{\pi} v^{m-2}{ }_{1} F_{2}\left(\frac{m-2}{4} ; \frac{m+2}{4}, 1+\frac{m}{4} ; \frac{v^{4}}{64}\right)}{8(m-2) \Gamma\left(1+\frac{m}{4}\right)} \\
& -\frac{v^{m-4}{ }_{2} F_{3}\left(1, \frac{m-4}{4} ; \frac{1}{2}, \frac{m+2}{4}, \frac{m}{4} ; \frac{v^{4}}{64}\right)}{(m-4) \Gamma\left(\frac{m+2}{4}\right)} \\
& -\frac{2^{(3 m / 2)-7} \Gamma\left(\frac{m-2}{4}\right)}{\sin (m \pi / 4)} .
\end{aligned}
$$

If $m=2$ or $m=6$, then even the $v$-integration remaining in Eq. (77) can be performed to determine $j_{1}(m)$ analytically because the scaling function $\Phi_{m, d^{*}}$ reduces to an elementary function. ${ }^{14,15}$ Analogous statements apply to the integrals $J_{2,3}(m), I^{(1)}(m)$, and $I^{(2)}(m)$ (cf. Appendix E). One obtains

$$
j_{1}(2)=1-\frac{\ln 3}{2},
$$

$$
j_{1}(6)=-\frac{2}{3}+2 \ln \frac{27}{16}
$$

$$
\begin{gathered}
{\left[b_{m} \frac{2}{3} \frac{J_{2,3}(2)}{6-m}\right]_{m=2}=\frac{1}{54},} \\
{\left[b_{m} \frac{2}{3} \frac{J_{2,3}(6)}{6-m}\right]_{m \rightarrow 6}=\frac{9 \ln (4 / 3)-2}{27},}
\end{gathered}
$$

$$
\begin{aligned}
b_{2} I^{(1)}(2)= & \frac{1}{16}\left[\operatorname{Li}_{2}(2 / 3)-\operatorname{Li}_{2}(1 / 4)-\ln (4 / 3) \ln 2\right] \\
& +\frac{\pi^{2}}{192}-\frac{1}{12} \ln (3 / 2) \\
& {\left[b_{m} I^{(1)}(m)\right]_{m \rightarrow 6}=\frac{\ln (4 / 3)}{6}, }
\end{aligned}
$$

$$
b_{2} I^{(2)}(2)=\frac{\pi^{2}+\ln (4 / 3)[3 \ln (4 / 3)-8]+6 \operatorname{Li}_{2}(1 / 4)}{96}
$$

and

$$
\left[b_{m} I^{(2)}(m)\right]_{m \rightarrow 6}=\frac{1-\ln (4 / 3)}{6},
$$

where it should be noted that $b_{m} /(6-m)$ approaches the finite value $2^{14} \pi^{9} / 3$ as $m \rightarrow 6$.

The limit

$$
j_{1}(0+)=\lim _{m \rightarrow 0+} j_{1}(m)=\frac{1}{2}
$$

can be determined in a similar manner as the $m \rightarrow 0$ limits of the integrals $j_{\phi}, \ldots, j_{u}$ were in Ref. 15 . For other values of $m$, no analytical results for $j_{1}(m)$ and the other integrals are available. We therefore resorted to numerical means, proceeding in a manner analogous to the one described in appendix E of Ref. 15 (see Appendix E below). The resulting numerical values of $j_{1}(m), J_{2,3}(m)$, $I^{(1)}(m)$, and $I^{(2)}(m)$ are gathered in Table I, along with those for the (previously computed ${ }^{15}$ ) integrals $j_{\phi}(m)$, $j_{\sigma}(m), j_{\rho}(m), j_{u}(m)$, and $J_{u}(m)$. 
TABLE I: Numerical values of the integrals $j_{\phi}(m), j_{\sigma}(m), j_{\rho}(m), j_{u}(m), J_{u}(m), j_{1}(m), 100 \times \frac{2}{3} \frac{b_{m}}{6-m} J_{2,3}(m), 10 \times b_{m} I^{(1)}(m)$, and $10 \times b_{m} I^{(2)}(m)$

\begin{tabular}{|c|c|c|c|c|c|c|}
\hline$m$ & 1 & 2 & 3 & 4 & 5 & 6 \\
\hline$j_{\phi}(m)$ & $1.642(9)$ & 1.33333 & $1.055(6)$ & $0.803(7)$ & $0.57(4)$ & 0.36521 \\
\hline$j_{\sigma}(m)$ & $1.339(4)$ & 4.74074 & $10.804(3)$ & $20.067(7)$ & $32.95(4)$ & 49.77778 \\
\hline$j_{\rho}(m)$ & $0.190(6)$ & 0.88889 & $1.999(9)$ & $3.464(1)$ & $5.23(4)$ & 7.26958 \\
\hline$j_{u}(m)$ & $-0.203(7)$ & -0.40547 & $-0.624(2)$ & $-0.880(1)$ & $-1.21(1)$ & -1.72286 \\
\hline$J_{u}(m)$ & $0.383(8)$ & 0.28768 & $0.200(8)$ & $0.119(8)$ & $0.04(3)$ & -0.02971 \\
\hline$j_{1}(m)$ & 0.47289 & 0.45069 & 0.43092 & 0.41273 & 0.39577 & 0.37983 \\
\hline$\frac{2}{3} \frac{100 b_{m}}{6-m} J_{2,3}(m)$ & 1.66642 & 1.85185 & 1.98142 & 2.07349 & 2.13819 & 2.18198 \\
\hline $10 b_{m} I^{(1)}(m)$ & $0.3644(5)$ & 0.40504 & $0.4337(1)$ & 0.45438 & $0.4690(0)$ & 0.47947 \\
\hline $10 b_{m} I^{(2)}(m)$ & $0.882(2)$ & 0.98150 & $1.05(4)$ & $1.110(7)$ & $1.15(3)$ & 1.18720 \\
\hline
\end{tabular}

\section{RG equations and scaling}

Exploiting the relation (73) between the bare and renormalized cumulants in a standard fashion yields the $\mathrm{RG}$ equations

$$
\begin{array}{r}
{\left[\mathcal{D}_{\mu}^{(\infty)}+\frac{(N+M) \eta_{\phi}}{2}+\frac{M \eta_{1, \infty}}{2}\right] G_{\infty, \text { ren }}^{(N, M)}} \\
=\delta_{M, 2}^{N, 0}\left(\mu \mathcal{R}_{1}-\sigma^{1 / 2} \mathcal{R}_{2} \partial_{\alpha} \partial_{\alpha}\right) \delta\left(\boldsymbol{r}_{12}\right)
\end{array}
$$

with

$$
\begin{gathered}
\mathcal{D}_{\mu}^{(\infty)} \equiv \mu \partial_{\mu}+\sum_{\wp=u, \sigma, \tau, \rho} \beta_{\wp} \partial_{\wp} \\
\left.\mathcal{R}_{1}(\mu, \rho, u) \equiv Z_{\phi}^{-1} Z_{1, \infty}^{-1} \partial_{\mu}\right|_{0}\left[Z_{\phi} Z_{1, \infty} \mu\left(C_{0}+\rho C_{1}\right)\right]
\end{gathered}
$$

and

$$
\left.\mathcal{R}_{2}(\sigma, u) \equiv \sigma^{-1 / 2} Z_{\phi}^{-1} Z_{1, \infty}^{-1} \mu \partial_{\mu}\right|_{0}\left[Z_{\phi} Z_{1, \infty} \sigma^{1 / 2} C_{2}\right],
$$

where $\eta_{1, \infty}(u)$ is defined by Eq. (29) with $\wp=(1, \infty)$.

The RG equation for $G_{\infty}^{(0,2)}$,ren is inhomogeneous because of the additive counterterm in Eq. (72) and the implied additive term in the reparametrization relation (73). If $C_{0}, C_{1}$ and $C_{2}$ are determined by minimal subtraction of poles (so that $C_{1}$ and $C_{2}$ are Laurent series in $\epsilon$ with a vanishing regular part while $C_{0}=0$ ), then the uv finiteness of $\mathcal{R}_{1}$ and $\mathcal{R}_{2}$ together with the familiar expressions (33) and (35) for $\beta_{u}$ and the RG functions $\eta_{\iota}$ imply that Eqs. (91) and (92) simplify to

$$
\mathcal{R}_{1}=-\rho u \partial_{u} \operatorname{Res}_{\epsilon=0} C_{1}(u, \epsilon), \quad \mathcal{R}_{2}=-u \partial_{u} \operatorname{Res}_{\epsilon=0} C_{2}(u, \epsilon) .
$$

Thus, to order $u^{2}, \mathcal{R}_{2}$ is simply given by $-2 \epsilon$ times the term $\propto u^{2} / \epsilon$ on the right-hand side of Eq. (75). For the exponent function $\eta_{1, \infty}(u)$ we find from Eq. (74) the result

$$
\eta_{1, \infty}(u)=-\frac{n+2}{6} u\left\{1+u\left[j_{1}(m)-J_{u}(m)\right]\right\}+O\left(u^{3}\right) .
$$

We are now ready to exploit the RG equations (89) by an appropriate generalization of the analysis given in
Ref. 14. Owing to the $\rho$ dependent term of the function $\beta_{\tau}$ [cf. the set of equations (33)], the flow equations (40) for $\bar{\tau}$ and $\bar{\rho}$ are coupled. We therefore introduce a nonlinear scaling field

$$
g_{\tau}(\tau, \rho, u)=\tau+c_{\rho^{2}}^{\tau}(u) \rho^{2}
$$

such that the associated running variable is a solution to

$$
\ell \frac{d}{d \ell} \bar{g}_{\tau}(\ell)=-\left[2+\eta_{\tau}(\bar{u})\right] \bar{g}_{\tau}(\ell), \quad \bar{g}_{\tau}(1)=g_{\tau}
$$

Substituting the ansatz (95) into the flow equations (40) leads to the condition

$$
\beta_{u}(\bar{u}, \epsilon) \frac{\partial c_{\rho^{2}}^{\tau}(\bar{u})}{\partial \bar{u}}+b_{\tau}(\bar{u})=\left[\eta_{\tau}(\bar{u})-2 \eta_{\rho}(\bar{u})\right] c_{\rho^{2}}^{\tau}(\bar{u})
$$

which is easily solved for $\bar{u}$ near $u^{*}$ to obtain

$$
c_{\rho^{2}}^{\tau}(\bar{u})=\frac{b_{\tau}^{*}}{\eta_{\tau}^{*}-2 \eta_{\rho}^{*}}+O\left(\bar{u}-u^{*}\right) .
$$

Since we shall ignore corrections to scaling $\bar{u}-u^{*}$ below, it will be sufficient to keep the first term on the righthand side. Our one-loop result $(39)$ for $b_{\tau}(u)$ yields for it:

$$
c_{\rho^{2}}^{\tau}\left(u^{*}\right)=\frac{b_{\tau}^{*}}{\eta_{\tau}^{*}-2 \eta_{\rho}^{*}}=-\frac{m}{8}+O\left(u^{*}\right) .
$$

Let us define the RG trajectory integrals

$$
\begin{aligned}
E_{\iota}[\bar{u}, u] & =\exp \left\{\int_{u}^{\bar{u}(\ell)} d x \frac{\eta_{\iota}^{*}-\eta_{\iota}(x)}{\beta_{u}(x)}\right\}, \\
\iota & =\phi, \sigma, \rho, \tau,(1, \infty),
\end{aligned}
$$

and the nonuniversal constants

$$
E_{\iota}^{*}(u) \equiv E_{\iota}\left(u^{*}, u\right), \iota=\phi, \sigma, \rho, \tau,(1, \infty),
$$

they approach in the infrared limit $\ell \rightarrow 0$. Then the solutions to the flow equations (40) and (96) for $\bar{\sigma}, \bar{\rho}$, and $\bar{g}_{\tau}$ can be written as

$$
\bar{\sigma}(\ell)=\ell^{-\eta_{\sigma}^{*}} E_{\sigma}[\bar{u}(\ell), u] \sigma \underset{\ell \rightarrow 0}{\approx} \ell^{-\eta_{\sigma}^{*}} E_{\sigma}^{*}(u) \sigma
$$




$$
\bar{\rho}(\ell)=\ell^{-\left(1+\eta_{\rho}^{*}\right)} E_{\rho}[\bar{u}(\ell), u] \rho \underset{\ell \rightarrow 0}{\approx} \ell^{-\varphi / \nu_{l 2}} E_{\rho}^{*}(u) \rho,
$$

and

$$
\bar{g}_{\tau}(\ell)=\ell^{-\left(2+\eta_{\tau}^{*}\right)} E_{\tau}[\bar{u}(\ell), u] g_{\tau} \underset{\ell \rightarrow 0}{\approx} \ell^{-1 / \nu_{l 2}} E_{\tau}^{*}(u) g_{\tau},
$$

where we have introduced the familiar correlation-length exponent

$$
\nu_{l 2} \equiv\left(2+\eta_{\tau}^{*}\right)^{-1}
$$

and the crossover exponent

$$
\varphi=\nu_{l 2}\left(1+\eta_{\rho}^{*}\right) .
$$

We choose $\ell \equiv \ell_{\tau}$ such that $\bar{g}_{\tau}\left(\ell_{\tau}\right)= \pm 1$ for $\pm g_{\tau}>0$, consider the limit $g_{\tau} \rightarrow 0 \pm$, and introduce the scaling lengths ${ }^{45}$

$$
\xi_{l 2} \equiv \mu^{-1} / \ell_{\tau} \approx \mu^{-1}\left[E_{\tau}^{*}(u)\left|g_{\tau}\right|\right]^{-\nu_{l 2}}
$$

and

$$
\xi_{l 4} \equiv\left[\frac{\bar{\sigma}\left(\ell_{\tau}\right)}{\mu^{2} \ell_{\tau}^{2}}\right]^{1 / 4} \approx \mu^{-1 / 2}\left[E_{\tau}^{*}(u)\left|g_{\tau}\right|\right]^{-\nu_{l 4}},
$$

where $\nu_{l 4}$ denotes the correlation-length exponent

$$
\nu_{l 4} \equiv \theta \nu_{l 2}=\frac{2+\eta_{\sigma}^{*}}{4\left(2+\eta_{\tau}^{*}\right)} .
$$

The RG equations (89) can now be solved in a straightforward manner via characteristics. It is convenient to utilize instead of $\tau$ the nonlinear scaling field $g_{\tau}$, considering the cumulants $G_{\infty, \text { ren }}^{(N, M)}$ as functions of $\rho, g_{\tau}$, and $u$. Upon substituting the trajectory integrals (100) by their fixed-point values (101) and the running interaction constants $\bar{\sigma}, \bar{\rho}$, and $\bar{g}_{\tau}$ by their asymptotic expressions displayed on the far right of Eqs. (102)-(104), one finds that the $G_{\text {ren }}^{(N, M)}$ behave near the Lifshitz point as

$$
\begin{aligned}
G_{\infty, \text { ren }}^{(N, M)}\left(r_{\alpha}, r_{\beta}, z ; \rho, g_{\tau}, u, \sigma, \mu\right) & \\
\approx & {\left[\frac{\mu^{-\eta_{\phi}^{*}}}{E_{\phi}^{*}} \xi_{l 2}^{-\left(d-m-2+\eta_{\phi}^{*}\right)} \xi_{l 4}^{-m}\right] } \\
& \times\left[\frac{\mu^{-\eta_{1, \infty}^{*}}}{E_{1, \infty}^{*}} \xi_{l 2}^{-2-\eta_{1, \infty}^{*}}\right]^{M / 2} \\
& \times \mathcal{G}_{\infty, \pm}^{(N, M)}\left[\frac{r_{\alpha}}{\xi_{l 4}}, \frac{r_{\beta}}{\xi_{l 2}}, \frac{z}{\xi_{l 2}} ; E_{\rho}^{*} \rho\left(\mu \xi_{l 2}\right)^{\varphi / \nu_{l 2}}\right] .
\end{aligned}
$$

Here $r_{\alpha}$ and $r_{\beta}$ stand for the sets of all $\alpha$ and $\beta$ coordinates parallel to the surface, respectively, while $z$ represents the set of all coordinates perpendicular to it. Further, $\mathcal{G}_{\infty, \pm}^{(N, M)}$ is a universal scaling function, given by

$$
\begin{aligned}
& \mathcal{G}_{\infty, \pm}^{(N, M)}\left(r_{\alpha}, r_{\beta}, z ; \rho\right) \\
& \quad \equiv G_{\infty, \text { ren }}^{(N, M)}\left[r_{\alpha}, r_{\beta}, z ; \rho, g_{\tau}=1, u^{*}, 1,1\right] .
\end{aligned}
$$

In the special case of $G_{\infty, \text { ren }}^{(0,2)}\left(\boldsymbol{r}_{12}\right)$, the inhomogeneity implies a contribution $\propto \delta\left(\boldsymbol{r}_{12}\right)$ linear in $\rho$ we suppressed in Eq. (111), regarding $G_{\infty, \text { ren }}^{(0,2)}\left(\boldsymbol{r}_{12}\right)$ as a conventional function (rather than a distribution) with $\boldsymbol{r}_{12} \neq \mathbf{0}$. In momentum space this term yields a regular contribution. It reflects the fact that the momentum-dependent surface susceptibility $\chi_{11}(\boldsymbol{p})[\mathrm{cf}$. Eq. (52)] does not diverge at the ordinary transition and may be viewed as analog of the term $\propto \delta_{M, 2}^{N, 0}$ in Eq. (68).

Owing to the relations (68) between the bare cumulants $G^{(N, M)}$ in the limit $\stackrel{\circ}{c} \rightarrow \infty$ and $G_{\infty}^{(N, M)}$ the scaling forms (111) carry over to the behavior of the former at the LP ordinary transition. An immediate consequence is that the critical exponents at the LP ordinary transition can be expressed in terms of four independent bulk critical indices, e.g., the correlation exponent

$$
\eta_{l 2} \equiv \eta_{\phi}^{*}
$$

and the previously introduced anisotropy, correlationlength, and crossover exponents $\theta, \nu_{l 2}$, and $\varphi$ [cf. Eqs. (105) and (106)], and a single surface critical index. As the latter one can choose the critical exponent $\beta_{1}^{\text {ord }}$ governing the asymptotic dependence

$$
m_{1}(\tau, \rho=0) \underset{\tau \rightarrow 0-}{\sim}|\tau|^{\beta_{1}}
$$

of the surface magnetization $m_{1}$ at $\rho=0$ (and given values of the remaining interaction constants $c, u$, and $\lambda)$. From the behavior of $G_{\infty, \text { ren }}^{(0,1)}$ predicted by Eq. (111) one reads off that

$$
\beta_{1}^{\text {ord }}=\nu_{l 2}\left[d+\eta_{l 2}+\eta_{1, \infty}^{*}+m(\theta-1)\right] / 2 .
$$

\section{Boundary operator expansion}

In the above derivation of the scaling behavior of the cumulants $G^{(N, M)}$ at the ordinary transition we relied on the large- $\stackrel{c}{c}$ form (68) of these functions. An alternative way to arrive at the same conclusions, which gives further insight, is the use of the boundary operator expansion (BOE). ${ }^{28,37}$ Since both the bare and renormalized theories satisfy Dirichlet boundary conditions for $\stackrel{\circ}{c}=\infty$ and $c=\infty$, there is no term $\propto \phi^{\mathfrak{B}}$ contributing to the BOE of $\phi(\boldsymbol{r}, z)$. Just as in the CP case, the leading operator should be $\partial_{n} \phi$. Hence we write

$$
\phi_{\text {ren }}(\boldsymbol{r}, z) \underset{z \rightarrow 0}{\approx} C_{\infty}(z)\left(\partial_{n} \phi\right)_{\text {ren }}(\boldsymbol{r}) .
$$

Consistency with the RG equations (89) requires that the function $C_{\infty}(z)$ must satisfy the RG equation

$$
\left(\mathcal{D}_{\mu}^{(\infty)}-\eta_{1, \infty} / 2\right) C_{\infty}(z)=0 .
$$

Solving this (with $\rho$ set to zero) yields a short-distance singularity for $z \ll \xi_{l 2}$ of the form

$$
C_{\infty}(z) \approx \operatorname{const} \mu^{-1}(\mu z)^{1+\eta_{1, \infty}^{*} / 2} .
$$


By analogy to the CP case, the exponent is governed by the difference between

$$
\beta_{l}=\nu_{l 2}\left[d-2+\eta_{l 2}+m(\theta-1)\right] / 2,
$$

the critical index of the bulk order parameter $m_{\mathrm{b}}=$ $\left\langle\left.\phi\right|_{z=\infty}\right\rangle$, and $\beta_{1}^{\text {ord }}$; we have

$$
\left(\beta_{1}^{\text {ord }}-\beta_{l}\right) / \nu_{l 2}=1+\eta_{1, \infty}^{*} / 2 .
$$

\section{E. Exact critical exponent of the surface energy density}

In the foregoing subsections IV A-IV D we have shown that the critical exponents of the cumulants $G^{(N, M)}$ at the ordinary transitions can all be expressed in terms of four bulk critical indices (e.g., $\eta_{l 2}, \theta, \nu_{l 2}, \varphi$ ) and a single surface critical index such as $\beta_{1}^{\text {ord }}$. We now turn to a discussion of the behavior of the surface energy density

$$
\mathcal{E}_{1} \equiv\left\langle\left[\boldsymbol{\phi}^{\mathfrak{B}}(\boldsymbol{r})\right]^{2} / 2\right\rangle
$$

at the LP ordinary transition.

The surface energy density at the CP ordinary transition is a well-known example of a local boundary density whose critical exponents are expressible in terms of bulk exponents: Its leading thermal singularity is of the same form $|\tau|^{2-\alpha}$ as the bulk free energy. ${ }^{46-49}$ Our aim here is to show that the same holds true for the surface energy density at the LP ordinary transition.

A particularly easy way to obtain this result is to argue along the lines of Ref. 49 and consider a finite system that extends in the $z$-direction from $z=-L_{2}$ to $z=L_{1}$, has cross-sectional area $A$, and different values $\stackrel{\circ}{1}_{1}, \stackrel{\circ}{\rho}_{1}$ and $\stackrel{\circ}{\tau}_{2}$, $\stackrel{\circ}{\rho}_{2}$ in the regions $\mathfrak{V}_{1}$ with $z>0$ and $\mathfrak{V}_{2}$ with $z<0$, respectively. Its Hamiltonian reads

$$
\mathcal{H}_{2}=\int_{\mathfrak{V}_{1}} d^{d} x \mathcal{L}_{\mathrm{b}}\left(\boldsymbol{x} ; \stackrel{\circ}{1}_{1}, \stackrel{\circ}{\rho}_{1}\right)+\int_{\mathfrak{V}_{2}} d^{d} x \mathcal{L}_{\mathrm{b}}\left(\boldsymbol{x} ; \stackrel{\circ}{\tau}_{2}, \stackrel{\circ}{\rho}_{2}\right),
$$

where $\mathcal{L}_{\mathrm{b}}(\boldsymbol{x} ; \stackrel{\circ}{\tau}, \stackrel{\circ}{\rho})$ is the bulk density $(2)$. We now choose values of $\stackrel{\circ}{2}_{2}$ and $\stackrel{\circ}{\rho}_{2}$ such that the bulk at large $-z>0$ is not critical and in the disordered phase when the thermodynamic limit is taken. ${ }^{50}$ Since the half-space $z<0$ for the chosen values of $\stackrel{\circ}{2}_{2}$ and $\stackrel{\circ}{\rho}_{2}$ is neither critical nor has no long-range order, its net effect is to generate modified effective short-ranged $O(n)$ invariant interactions at the interface $z=0$. The boundary critical behavior which occurs at this interface as $\stackrel{\circ}{\tau}_{1} \rightarrow \stackrel{\circ}{\mathrm{LP}}_{\text {and }} \stackrel{\circ}{\rho}_{1} \rightarrow \stackrel{\circ}{\rho}_{\mathrm{LP}}$ should be in the universality class of the LP ordinary transition.

Suppose the interface is displaced a small distance $\Delta L$ downwards, so that the heights $L_{1}$ and $L_{2}$ of the regions $\mathfrak{V}_{1}$ and $\mathfrak{V}_{2}$ increase and decrease by $\Delta L$, respectively. The change of total free energy per volume $A \Delta L$ caused by this displacement in the limit $A \rightarrow \infty$ and $L_{1}, L_{2} \rightarrow \infty$ becomes

$$
\begin{aligned}
& f_{\mathrm{b}}\left(\stackrel{\circ}{\tau}_{1}, \stackrel{\circ}{\rho}_{1}\right)-f_{\mathrm{b}}\left(\stackrel{\circ}{\tau}_{2}, \stackrel{\circ}{\rho}_{2}\right) \\
& =\left(\stackrel{\circ}{\tau}_{1}-\stackrel{\circ}{\tau}_{2}\right) \mathcal{E}_{1}+\frac{\stackrel{\circ}{\rho}_{1}-\stackrel{\circ}{\rho}_{2}}{2} \sum_{\alpha=1}^{m}\left\langle\left(\partial_{\alpha} \phi^{\mathfrak{B}}\right)^{2}\right\rangle .
\end{aligned}
$$

Here the left-hand side is simply the change of bulk free energy per unit volume caused by the alteration of values $\left(\stackrel{\circ}{\tau}_{2}, \stackrel{\circ}{\rho}_{2}\right) \rightarrow\left(\stackrel{\circ}{\tau}_{1}, \stackrel{\circ}{\rho}_{1}\right)$ within the region $-\Delta L \leq z \leq 0$. The right-hand side follows by expressing this change in terms of

$$
\Delta \mathcal{H}=\int_{0}^{\Delta L} d z \int d A\left[\frac{\stackrel{\circ}{\tau}_{1}-\stackrel{\circ}{\tau}_{2}}{2} \phi^{2}+\frac{\stackrel{\circ}{\rho}_{1}-\stackrel{\circ}{\rho}_{2}}{2} \sum_{\alpha=1}^{m}\left(\partial_{\alpha} \phi\right)^{2}\right],
$$

the corresponding change of the Hamiltonian, as $-(A \Delta L)^{-1} \ln \left\langle e^{-\Delta \mathcal{H}}\right\rangle \approx\langle\Delta \mathcal{H}\rangle /(A \Delta L)$, where $\langle$.$\rangle indi-$ cates a thermal average with the Boltzmann factor $e^{-\mathcal{H}_{2}}$ of the original unperturbed system. For small $\Delta L$ we have $\int_{0}^{\Delta L} d z f(z) \approx f(0) \Delta L$. Assuming translational invariance parallel to the interface then yields the righthand side of Eq. (122). (This assumption excludes situations in which the order parameter varies along the interface. In such cases of broken translational invariance along the interface the right-hand side of the identity (122) must be replaced by its spatial average $A^{-1} \int d A \ldots$ over the interface.)

For small deviations $\stackrel{\circ}{\tau}_{1}-\stackrel{\circ}{\tau}_{\mathrm{LP}}$ and $\stackrel{\circ}{\rho}_{1}-\stackrel{\circ}{\rho} \mathrm{LP}_{\text {from the }}$ LP, i.e., small values of the scaling fields $g_{\tau}$ [cf. Eq. (95)] and $\rho$, the first term on the right-hand side of Eq. (122) behaves as

$$
f_{\mathrm{b}}\left(\stackrel{\circ}{\tau}_{1}, \stackrel{\circ}{\rho}_{1}\right) \approx\left|g_{\tau}\right|^{2-\alpha_{l}} \mathcal{F}_{ \pm}\left(\rho\left|g_{\tau}\right|^{-\varphi}\right)+\text { reg } .
$$

Here "reg" stands for regular contributions, and the plus and minus signs of the subscript \pm refer to the cases $g_{\tau}>0$ and $g_{\tau}<0$ respectively.

Noting that $\mathcal{F}(0) \neq 0$, we can equate the leading singular parts of the identity (122) to obtain the asymptotic behavior

$$
\begin{array}{r}
\left(\stackrel{\circ}{\mathrm{LP}}-\stackrel{\circ}{\tau}_{2}\right) \mathcal{E}_{1}^{\operatorname{sing}}+\frac{\stackrel{\circ}{\rho} \mathrm{LP}-\stackrel{\circ}{\rho}_{2}}{2} \sum_{\alpha=1}^{m}\left\langle\left(\partial_{\alpha} \phi^{\mathfrak{B}}\right)^{2}\right\rangle^{\text {sing }} \\
\approx \mathcal{F}_{ \pm}(0)|\tau|^{2-\alpha_{l}}
\end{array}
$$

for $\tau \rightarrow 0 \pm$ at $\rho=0$. Hence one of the two surface quantities on the left-hand side must contain the singularity on the right-hand side.

The critical line $\stackrel{\circ}{\tau}_{c}=f_{\mathrm{CL}}(\stackrel{\circ}{\rho})$ is not a straight line in the vicinity of the LP. On the section separating the disordered phase from the modulated ordered one, its asymptotic behavior near the LP is governed by the crossover exponent $\varphi$, i.e., $\stackrel{\circ}{\tau}_{c}-\stackrel{\circ}{\tau}_{\mathrm{LP}} \sim\left(\stackrel{\circ}{\rho}-\stackrel{\circ}{\rho}_{\mathrm{LP}}\right)^{1 / \varphi}$. Nevertheless, choices of the point $\left(\stackrel{\circ}{\tau}_{2}, \stackrel{\circ}{\rho}_{2}\right)$ in the disordered phase with $\stackrel{\circ}{\rho}_{2}=\stackrel{\circ}{\rho}_{\mathrm{LP}}$ and $\stackrel{\circ}{\tau}_{2} \neq \stackrel{\circ}{\tau} \mathrm{LP}_{\mathrm{L}}$ should be possible. Since for such choices only the term proportional to the surface energy density $\mathcal{E}_{1}$ contributes on the left-hand side of Eq. (125), it is clear that $\mathcal{E}_{1}$ must have the bulk free-energy singularity on the right-hand side.

This result means that the scaling dimension $\Delta\left[\left(\phi^{\mathfrak{B}}\right)^{2}\right]$ of the surface energy density operator $\left(\phi^{\mathfrak{B}}\right)^{2}$ coincides with that of the volume $\int d V$, i.e.,

$$
\Delta\left[\left(\phi^{\mathfrak{B}}\right)^{2}\right]=d+\theta(m-1) .
$$


As usual, the amplitudes $\mathcal{F}_{ \pm}(0)$ are nonuniversal, but the ratio $\mathcal{F}_{+}(0) / \mathcal{F}_{-}(0)$ is a universal bulk amplitude ratio. The corresponding amplitude ratio of $\mathcal{E}_{1}$ must have the same value.

There is an instructive alternative way to confirm this result. Knowing that the ordinary fixed point $\mathcal{P}_{\text {ord }}^{*}$ is located at $c=\infty$, one can study the effect of the Hamiltonian's boundary term $(\stackrel{\circ}{c} / 2) \int_{\mathfrak{B}} \phi^{2}$ for large but finite $\stackrel{\circ}{c}$ to leading order in $1 / \stackrel{\circ}{c}$.

Expanding the free propagator $(7)$ in powers of $1 /{ }^{\circ} \boldsymbol{p}$ gives

$$
\begin{aligned}
& \hat{G}\left(\boldsymbol{p} ; z, z^{\prime}\right)-\hat{G}_{\mathrm{D}}\left(\boldsymbol{p} ; z, z^{\prime}\right) \\
& \quad=\stackrel{\circ}{c}_{\boldsymbol{p}}^{-1} e^{-\stackrel{\circ}{\boldsymbol{p}}_{\boldsymbol{p}}\left(z-z^{\prime}\right)}+O\left(\stackrel{\circ}{\boldsymbol{p}}^{-2}\right) \\
& =\stackrel{\circ}{c}_{\boldsymbol{p}}^{-1}\left(\hat{G}_{\mathrm{D}} \overleftarrow{\partial}_{n}\right)(\boldsymbol{p} ; z, 0)\left(\partial_{n} \hat{G}_{\mathrm{D}}\right)\left(\boldsymbol{p} ; 0, z^{\prime}\right)+O\left(\stackrel{\circ}{c}_{\boldsymbol{p}}^{-2}\right)
\end{aligned}
$$

where $\overleftarrow{\partial}_{n}$ acts to the left. More generally, one notices that the expansion of the free propagator in powers of $1 /{ }^{\circ} \boldsymbol{p}$ in the present $m \neq 0$ case is completely analogous to the expansion of its $m=0$ counterpart in powers of $1 / \stackrel{\circ}{c}$, with identical expansion coefficients [cf. Eq. (11) of Ref. 47].

If we restrict ourselves to first order in $\stackrel{\circ}{ }^{-1}$, we can make the replacement $\stackrel{\circ}{p}_{p}^{-1} \rightarrow \stackrel{\circ}{c}^{-1}$. In analogy with the $m=0$ case the expansion of the $N$-point cumulant to first order in $\stackrel{\circ}{c}^{-1}$ therefore becomes

$$
G^{(N, 0)}=G_{\infty}^{(N, 0)}+\stackrel{\circ}{c}^{-1} G_{\infty}^{(N, 0 ; 1)}+O\left(\stackrel{\circ}{c}^{-2}\right),
$$

where $G_{\infty}^{(N, 0 ; 1)}$ means a cumulant involving $N$ fields $\phi_{a}$ and a single insertion of the boundary operator $\int_{\mathfrak{B}} d A\left(\partial_{n} \phi\right)^{2} / 2$, evaluated at $\stackrel{\circ}{c}=\infty$ and $\stackrel{\circ}{\lambda}=0$. Proceeding as in Appendix $\mathrm{C}$ of Ref. 47, one can easily derive the relation

$$
\sum_{i=1}^{N} \partial_{z_{i}} G_{\infty}^{(N, 0)}\left(\boldsymbol{x}_{1}, \ldots, \boldsymbol{x}_{N}\right)=G_{\infty}^{(N, 0 ; 1)}\left(\boldsymbol{x}_{1}, \ldots, \boldsymbol{x}_{N}\right)
$$

where $z_{i}$ is the $z$-component of $\boldsymbol{x}_{i}$. Since the renormalization factor $Z_{\phi}^{-N / 2}$ of the cumulant on the left-hand side must also renormalize the one on the right-hand side, the inserted boundary operator needs no renormalization. Thus the associated interaction constant $\sim i^{-1}$ must scale naively as $1 / \mu$ at the ordinary fixed point. Noting that the RG eigenexponent $y_{1}$ of a boundary scaling field $g_{1}$ and the scaling dimension $\Delta\left[\mathcal{O}_{1}\right]$ of a local boundary operator $\mathcal{O}_{1}$ (in the case of the parallel surface orientation considered in this paper) are related via

$$
\Delta\left[\mathcal{O}_{1}\right]=d-1+m(\theta-1)-y_{1},
$$

one immediately concludes that the scaling dimension of $\Delta\left[\left(\partial_{n} \phi\right)^{2}\right]$ of the inserted operator $\left(\partial_{n} \phi\right)^{2} / 2$, which represents the surface energy density at the ordinary transition, is indeed given by (126).

The above findings indicate that the boundary operator with smallest scaling dimension contributing to the
BOE of the local energy density operator $\phi^{2}(\boldsymbol{x})$ (besides the one-operator $\mathbb{1}$ ) is $T_{z z}^{\mathfrak{B}}$, the $z z$-component of the stress-energy tensor $T_{\mu \nu}$, taken at the boundary (cf., e.g., Ref. 51).

\section{CRITICAL EXPONENTS OF THE ORDINARY TRANSITION}

\section{A. Definition of surface correlation exponents}

In order to characterize the power-law decay of the pair correlation function $G^{(2,0)}\left(\boldsymbol{x}_{1}, \boldsymbol{x}_{2}\right)$ of semi-infinite systems at a critical point in the limits where the separation $\boldsymbol{x}_{1}-\boldsymbol{x}_{2}$ increases parallel or perpendicular to the surface, one conventionally introduces analogs of the usual correlation exponent $\eta$ termed $\eta_{\|}$and $\eta_{\perp}$, respectively. In the case of anisotropic scale invariant systems with boundaries we must be more careful because distances along different axes scale differently and the orientation of the surface matters.

Clearly, distinct sets of surface correlation exponents $\eta_{l 2, \|}, \eta_{l 2, \perp}$, and $\eta_{l 4, \|}, \eta_{l 4, \perp}$, could be introduced in analogy with the bulk correlation exponents $\eta_{l 2}$ and $\eta_{l 4}$. However, this is unnecessary since the "l4 exponents" are simply related to their " $l 2$ counterparts" via the anisotropy exponent $\theta$, just as the introduction of $\eta_{l 4}$ could be avoided by writing the exponent $4-\eta_{l 4}$ as $\theta\left(2-\eta_{l 2}\right)$. In order to define the exponents $\eta_{\|} \equiv \eta_{l 2, \|}$ and $\eta_{\perp} \equiv \eta_{l 2, \perp}$, consider the pair correlation function $G^{(2,0)}\left(\boldsymbol{r} ; z, z^{\prime}\right)$ at the Lifshitz point between two points $\boldsymbol{x}=(\boldsymbol{r}, z)$ and $\boldsymbol{x}^{\prime}=\left(\mathbf{0}, z^{\prime}\right)$, and let us introduce

$$
\check{r} \equiv \sqrt{r_{\alpha} r_{\alpha}} \quad \text { and } \quad R \equiv \sqrt{r_{\beta} r_{\beta}},
$$

the lengths of the components $\left(r_{\alpha}\right)$ and $\left(r_{\beta}\right)$ of their parallel separation $r$. In the limits $z \rightarrow \infty, R \rightarrow \infty$ or $\check{r} \rightarrow \infty$, with $z^{\prime}$ and the respective other distances kept fixed, this function decays as

$$
G^{(2,0)}\left(\boldsymbol{r} ; z, z^{\prime}\right) \sim \begin{cases}z^{-\left[d-2+m(\theta-1)+\eta_{\perp}\right]}, & z \rightarrow \infty, \\ R^{-\left[d-2+m(\theta-1)+\eta_{\|}\right]}, & R \rightarrow \infty, \\ \check{r}^{-\left[d-2+m(\theta-1)+\eta_{\|}\right] / \theta} & , \check{r} \rightarrow \infty .\end{cases}
$$

These relations translate into the small-momentum behavior

$$
\chi_{11}(\boldsymbol{p})^{\operatorname{sing}} \sim \begin{cases}p^{\eta_{\|}-1} & \text { for }\left(p_{\alpha}\right)=(0), \\ p^{\left(\eta_{\|}-1\right) / \theta} & \text { for }\left(p_{\beta}\right)=(0),\end{cases}
$$

and $^{52}$

$$
\chi_{1}(\boldsymbol{p}) \sim \begin{cases}p^{\eta_{\perp}-2} & \text { for }\left(p_{\alpha}\right)=(0), \\ p^{\left(\eta_{\perp}-2\right) / \theta} & \text { for }\left(p_{\beta}\right)=(0),\end{cases}
$$

of the local surface susceptibility (52) and the layer surface susceptibility

$$
\chi_{1}(\boldsymbol{p})=\int_{0}^{\infty} \hat{G}^{(1,1)}(\boldsymbol{p} ; z) d z,
$$


respectively.

The large- $\stackrel{\circ}{c}$ behavior (68) in conjunction with the scaling forms (111) implies that

$$
\eta_{\|}^{\text {ord }}=2+\eta_{l 2}+\eta_{1, \infty}^{*} .
$$

The corresponding result for $\eta_{\perp}^{\text {ord }}$ is equivalent to the scaling law

$$
\eta_{\perp}=\left(\eta_{l 2}+\eta_{\|}\right) / 2
$$

which is well known from the CP case. ${ }^{28,36,37,53}$

Let us also note that the decay law of $G^{(2,0)}$ (involving $\eta_{\perp}^{\text {ord }}$ ) displayed in the first line on the right-hand side of Eq. (132) carries over to the case where $\boldsymbol{x}$ moves away from the surface along an arbitrary direction not parallel to the surface. This can be shown as in the CP case, ${ }^{28,37}$ utilizing the BOE (115) and Eq. (117).

\section{B. $\epsilon$ expansion of surface critical exponents and estimates for three dimensions}

We now turn to the $\epsilon$ expansions of the critical exponents at the ordinary transition. Substituting the $\epsilon$ expansion (42) of the nontrivial root $u^{*}$ of $\beta_{u}$ into Eq. (94) yields

$$
\begin{aligned}
\eta_{1, \infty}^{*}= & \eta_{\|}^{\text {ord }}-\eta_{l 2}-2 \\
= & -\frac{n+2}{n+8} \epsilon-\frac{(n+2)^{2}}{(n+8)^{3}}\left[\frac{j_{\sigma}(m)}{16(m+2)}-\frac{j_{\phi}(m)}{2}\right. \\
& \left.+2 \frac{20+7 n}{n+2} J_{u}(m)+6 \frac{n+8}{n+2} j_{1}(m)\right] \epsilon^{2} \\
& +O\left(\epsilon^{3}\right) .
\end{aligned}
$$

For $m \rightarrow 0, m=2$, and $m=6$, the exactly known values $^{15}$

$$
\begin{gathered}
j_{\phi}(0)=2, \quad j_{\sigma}(0)=0, \quad J_{u}(0)=1 / 2, \\
j_{\phi}(2)=\frac{4}{3}, \quad j_{\sigma}(2)=\frac{128}{27}, \quad J_{u}(2)=\ln \frac{4}{3},
\end{gathered}
$$

and

$$
\begin{aligned}
j_{\phi}(6) & =\frac{8}{3}\left[1-3 \ln \frac{4}{3}\right], \quad j_{\sigma}(6)=\frac{448}{9}, \\
J_{u}(6) & =\frac{5}{6}-3 \ln \frac{4}{3}
\end{aligned}
$$

can be inserted into Eq. (138), along with those of $j_{1}$ given in Eqs. (88) and (80), to convert it into an analytical expression. Doing so one finds that the result (138) reduces in the limit $m \rightarrow 0$ to its established analog $36,37,54$ for the CP ordinary transition, Eq. (IV.35) of Ref. 37, as it should.

We can now replace $\eta_{l 2}$ in Eq. (138) by its known $O\left(\epsilon^{2}\right)$ expression [given in Eq. (85) of Ref. 14 and Eq. (61) of
Ref. 15] to obtain the $\epsilon$ expansion of $\eta_{\|}^{\text {ord }}$ to order $\epsilon^{2}$. The analogous "direct" series expansions to order $\epsilon^{2}$ of the surface exponents $\beta_{1}^{\text {ord }}, \eta_{\perp}^{\text {ord }}$, and the surface susceptibility exponents $\gamma_{1}^{\text {ord }}$ and $\gamma_{11}^{\text {ord }}$ follow in a similar fashion by combining the $\epsilon$-expansion results for the bulk exponents given in Eqs. (61)-(66) of Ref. 15 with ours for $\eta_{1, \infty}^{*}$ and the scaling relations (137),

$$
\begin{gathered}
\beta_{1}=\nu_{l 2}\left[d-2+\theta(m-1)+\eta_{\|}\right] / 2, \\
\gamma_{11}=\nu_{l 2}\left(1-\eta_{\|}\right),
\end{gathered}
$$

and

$$
\gamma_{1}=\nu_{l 2}\left(2-\eta_{\perp}\right)
$$

all of which are straightforward consequences of the scaling forms (111), the large- $\stackrel{c}{ }$ form of the cumulants $G^{(N, M)}$, and the short-distance behavior (117). They can be derived in much the same way as in the $m=0$ case, ${ }^{28,37}$ for the parallel orientation of the surface considered in this paper.

In Table II we have gathered the numerical estimates of various critical exponents of the LP ordinary transition one obtains for the uniaxial one-component case in three dimensions by evaluating their $\epsilon$ expansions to zeroth, first, and second order at $\epsilon=3 / 2$. The critical indices $\beta_{\mathrm{s}}$ and $\gamma_{\mathrm{s}}$, defined through the behavior $m_{\mathrm{s}} \sim|\tau|^{\beta_{\mathrm{s}}}$ and $\chi_{\mathrm{s}} \sim|\tau|^{-\gamma_{\mathrm{s}}}$ for $\tau \rightarrow 0, \rho=0$, of the excess magnetization

$$
m_{\mathrm{s}}=\int_{0}^{\infty} d z\left[G^{(1,0)}(z)-G^{(1,0)}(\infty)\right]
$$

and the excess susceptibility

$$
\chi_{\mathrm{s}}=\int_{0}^{\infty} d z \int_{0}^{\infty} d z^{\prime}\left[\hat{G}^{(2,0)}\left(\boldsymbol{p} ; z, z^{\prime}\right)-\hat{G}^{(2,0)}(\boldsymbol{p} ; \infty, \infty)\right]_{\boldsymbol{p}=\mathbf{0}}
$$

do not involve an independent surface exponent because they can be expressed in terms of bulk exponents as

$$
\beta_{\mathrm{s}}=\beta_{l}-\nu_{l 2}
$$

and

$$
\gamma_{\mathrm{s}}=\gamma_{l}+\nu_{l 2}
$$

We have included them in Table II to compare also them with the Monte Carlo results of Ref. 21.

The direct series expansions to $O\left(\epsilon^{2}\right)$ of the exponents listed in Table II are reasonably well behaved. We therefore expect the corresponding estimates to be fairly reliable. Yet for some of the series, Padé [1,1] approximants give significantly different values. In other, more favorable cases the differences are small. One example of this kind is $\beta_{1}^{\text {ord }}$. The Padé $[1,1]$ approximant and the solution to the scaling relation (119) yield

$$
\beta_{1}^{\text {ord }}(3,1,1) \simeq \begin{cases}0.683, & \text { Padé }[1,1], \\ 0.628, & \text { with bulk exponents },\end{cases}
$$


TABLE II: Estimated values of critical exponents at the LP ordinary transition in $d=3$ dimensions for $m=1$ and $n=1$. The values in the columns labeled "MFT", " $O(\epsilon)$ ", " $O\left(\epsilon^{2}\right)$ ", and "MC" correspond to mean field theory, to the estimates obtained by setting $\epsilon=3 / 2$ in the $\epsilon$ expansions to first and second order, and to Monte Carlo results, respectively.

\begin{tabular}{lrcrc}
\hline \hline & MFT & $O(\epsilon)$ & $O\left(\epsilon^{2}\right)$ & $\mathrm{MC}^{a}$ \\
\hline$\eta_{\|}^{\text {ord }}$ & 2 & 1.5 & 1.133 & - \\
$\eta_{\perp}^{\text {ord }}$ & 1 & 0.75 & 0.586 & - \\
$\beta_{1}^{\text {ord }}$ & 1 & 0.75 & 0.697 & $0.687(5)$ \\
$\gamma_{1}^{\text {ord }}$ & $1 / 2$ & 0.75 & 0.947 & $0.82(4)$ \\
$\gamma_{1,1}^{\text {ord }}$ & $-1 / 2$ & -0.375 & -0.212 & $-0.29(6)$ \\
$\beta_{\mathrm{s}}$ & 0 & -0.375 & -0.462 & $-0.46(3)$ \\
$\gamma_{\mathrm{s}}$ & $3 / 2$ & 1.875 & 2.106 & $1.98(8)$ \\
\hline \hline
\end{tabular}

${ }^{a}$ Taken from Ref. 21.

respectively. To obtain the second number, the "best estimates" $\beta_{l} \simeq 0.220$ and $\nu_{l 2} \simeq 0.746$ of Ref. 15 were inserted into Eq. (119) together with the direct series estimate $\eta_{1, \infty}^{*} \simeq-0.9060$. Both so-obtained numbers are approximately within $10 \%$ of the estimate given in Table II.

The exponent $\gamma_{1}^{\text {ord }}$ provides an example of a case in which the $d=3$ value predicted by the Padé approximant, namely $\gamma_{1}^{\text {ord }} \simeq 1.670$, deviates considerably from the direct series estimate. However, the relation $\gamma_{1}^{\text {ord }}=\nu_{l 2}\left(1-\eta_{l 2}-\eta_{1, \infty}^{*} / 2\right)$, applied with the just mentioned values of $\nu_{l 2}$ and $\eta_{1, \infty}^{*}$ and the "best estimate" $\eta_{l 2} \simeq 0.124$ of Ref. 15 , yields $\gamma_{1}^{\text {ord }} \simeq 0.99$, which is reasonably close to the direct series estimate (Table II). We therefore discard the Padé $[1,1]$ estimate in this case.

As can be seen from Table II, our estimates are in good agreement with Pleimling's ${ }^{21}$ Monte Carlo estimates. Normally the Monte Carlo results for $\beta_{1}$ are more accurate than for susceptibilities such as $\gamma_{1}$ and $\gamma_{11}$. It is therefore gratifying that the agreement is particularly good in the case of $\beta_{1}^{\text {ord }}$.

Of interest is also the case of the ordinary transition at the uniaxial Lifshitz point of the axial next-nearest neighbor XY model (ANNNXY model), with $n=2$ and $m=1$. This model was recently argued to describe the bulk critical behavior of Tb. ${ }^{55}$ To our knowledge, neither experimental nor theoretical results for the surface critical exponents at the corresponding ordinary transition have been published. On the other hand, Monte Carlo results for the bulk critical exponents of the threedimensional ANNNXY model have been obtained a long time ago. ${ }^{56,57}$ As discussed in Ref. 15 (see its tables 4 and 5), Selke's values ${ }^{57} \beta_{l}=0.2 \pm 0.02$ and $\gamma_{l}=1.5 \pm 0.1$ are in reasonable agreement with the $O\left(\epsilon^{2}\right)$ estimates $\beta_{l} \simeq 0.276$, and $\gamma_{l} \simeq 1.495$ obtained there. Inserting the latter together with the $O\left(\epsilon^{2}\right)$ result $\nu_{l 2} \simeq 0.757$ into Eqs. (147) and (148) yields $\beta_{s} \simeq-0.48$ and $\gamma_{s} \simeq 2.25$.

To obtain estimates of $\beta_{1}^{\text {ord }}$ for $d=3, m=1$, and $n=2$, we exploited our above results for its series expansion to order $\epsilon^{2}$. Direct extrapolation of this (truncated) series and Padé $[1,1]$ and $[0,2]$ approximants yielded the values $\beta_{1}^{\text {ord }} \simeq 0.72,0.71$, and 0.75 , respectively. Aside from experimental tests of these predictions, checks via high-precision Monte Carlo simulations would be very welcome.

\section{SUMMARY AND CONCLUSIONS}

We have investigated the critical behavior of semiinfinite $d$-dimensional systems at an $m$-axial bulk Lifshitz point. To this end we have considered systems whose bulk universality class is described by an $n$-component $\phi^{4}$ Hamiltonian with the bulk density (2). Assuming that the surface plane of the system is oriented parallel to all potential modulation axes in the modulated ordered phase, we have constructed appropriate "minimal" semiinfinite extensions of these continuum models with the following properties:

(a) They are compatible with the bulk universality class of Lifshitz critical behavior described by an infinitespace model whose Hamiltonian has the density (2).

(b) They are minimal in the sense that redundant and irrelevant surface terms compatible with the presumed $O(n)$ symmetry and short-range nature of the interactions and their surface-induced perturbations have been dropped.

(c) Taken at their respective RG fixed points, these models represent the (surface) universality classes of the associated ordinary, special, and extraordinary transitions.

The resulting contributions to the Hamiltonian (1) localized on the surface correspond to the surface density (3). They differ from the usual one known from the much studied $(m=0)$ case of a critical point, ${ }^{28}$ namely $(\stackrel{c}{c} / 2) \phi^{2}$, by a similar derivative term. We have shown that the latter is required for renormalizability of the model. Physically, this means that such a term, if initially absent, gets generated as short wave-length degrees of freedom are integrated out. The fluctuating boundary conditions implied by the Hamiltonian (1)-(3) are given in Eq. (4); in the $\boldsymbol{p} z$ representation they correspond to Robin boundary conditions with a momentum-dependent $\stackrel{\circ}{c}_{\boldsymbol{p}}$ [see Eqs. (4), (8) and (67)].

The boundary term $\propto \grave{\lambda}$ gives rise to a dimensionless interaction constant $\lambda$, on which the surface counterterms and renormalization functions depend for general values of $\stackrel{c}{c}$. We have clarified the fixed-point structure of the model, identifying the fixed points $\mathcal{P}_{\text {ord }}^{*}, \mathcal{P}_{\text {sp }}^{*}$, and $\mathcal{P}_{\text {ex }}^{*}$ describing the ordinary, special, and extraordinary transitions (cf. Fig 2), which are located at a nontrivial value (62) of $\lambda$.

In order to investigate the LP special transition, dealing with the $\lambda$ dependence cannot be avoided. However, as we have shown, this is possible in the case of the ordinary transition by taking the limit $\stackrel{\circ}{c} \rightarrow \infty$ from the outset. Just as in the $\mathrm{CP}(m=0)$ case, both the regularized bare and the renormalized theories obey Dirichlet 
boundary conditions if $\stackrel{\circ}{c}=\infty$, and the single required surface critical exponent $\beta_{1}^{\text {ord }}$ follows from the scaling dimension of $\partial_{n} \phi$. Likewise, the proofs ${ }^{47-49}$ for the CP case showing that the surface energy density at the ordinary transition has a leading singularity of the bulk free-energy form $\sim|\tau|^{2-\alpha}$ carry over to the present $m \neq 0$ one.

Performing a two-loop RG analysis of the LP ordinary transition for general values of $m$, we have been able to determine the $\epsilon$ expansions of the surface critical indices of this transition to second order in $\epsilon$. Extrapolations of these series expansions to $d=3$ dimensions yield values of the surface critical exponents for the uniaxial one-component case $m=n=1$ in good agreement with recent Monte Carlo results ${ }^{21}$ for the ANNNI model.

There are several obvious directions for extensions of the present work. First of all, the special transition should be analyzed, preferably by means of a two-loop RG analysis giving the $\epsilon$ expansions of its surface critical exponents to second order in $\epsilon$. From the results presented above, the $\epsilon$ expansions of the two independent surface critical exponents of this transition, the surface correlation exponent $\eta_{\|}^{\mathrm{sp}}$ and the surface crossover exponent $\Phi$, can be derived to first order in $\epsilon$ with moderate effort. ${ }^{58}$ However, a detailed investigation of the special transition requires more work, is beyond the scope of the present paper and left to a planned subsequent one. Suffice it here to say that for our $O(n)$ symmetric Hamiltonian with $n>1$, the existence of a surface-ordered phase at temperatures $T>0$ is ruled out for bulk dimensions $d \leq d_{*}^{O(n)}+1=3+m / 2$. Extrapolations of the $\epsilon$ expansions of the special transition's critical exponents to $d=3$ thus make sense only in the scalar case, $n=1$. For the interesting case $m=n=1$, Monte Carlo results for the exponent $\beta_{1}^{\text {sp }}$ of the ANNNI model are available ${ }^{21}$ which may be used to check the resulting $d=3$ extrapolation values.

A second important line of extension is to consider a surface orientation perpendicular to one of the potential modulation axes (the "perpendicular case" of the Introduction). This case is somewhat more difficult to handle than the parallel surface orientation treated in this paper. Since the $z$-direction then scales naively as $\mu^{-1 / 2}$ rather than as $1 / \mu$, more potentially dangerous boundary terms (whose coupling constants have nonnegative RG eigenexponents at the Gaussian fixed point) exist. Since the Landau-theory equations for the order parameter profile and the free propagator involve the fourth derivative $\partial^{4} / \partial z^{4}$, two boundary conditions rather than the single one, Eq. (4) or Eq. (6), are required and found. ${ }^{23}$ A proper study of this case beyond Landau theory ${ }^{19}$ also requires a careful identification of redundant surface operators.

Another obvious limitation of the present work is its lack of detailed investigations of the homogeneous and modulated ordered phases, the extraordinary transitions, as well as of profiles of the order parameter and other densities at the LP. The progress made here indicates that such studies, though technically demanding, should be possible, involving no fundamental new difficulties.

Unfortunately, there are, to our knowledge, yet no experimental results on surface critical behavior at bulk Lifshitz points available with which we could compare. Owing to the richness of the physics involved, detailed experimental studies would be very interesting. We hope that the progress made recently on the theoretical side will foster such experimental activities.

\section{Acknowledgments}

It is our pleasure to thank M. Shpot for helpful discussions. We gratefully acknowledge the support of this work provided by the Deutsche Forschungsgemeinschaft (DFG) - in its initial phase via Sonderforschungsbereich 237, in its final phase via DFG grant \# Di-378/3.

\section{APPENDIX A: LAURENT EXPANSION OF THE FEYNMAN INTEGRALS OF $\hat{G}_{\infty}^{(1,1)}$}

In this appendix, we briefly describe the calculation of the Laurent expansion of the one and two-loop graphs of the function $G_{\infty}^{(1,1)}\left(\boldsymbol{x}, \boldsymbol{x}^{\prime}\right)$ to the required orders in $\epsilon^{-1}$.

The Dirichlet propagator (66) to which the free propagator (7) reduces for $\stackrel{\circ}{c}=\infty$ can be written as

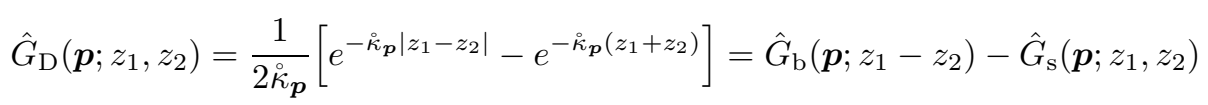

in the $\boldsymbol{p} z$ representation. The part depending on $\left|z_{1}-z_{2}\right|$ is the bulk propagator $\hat{G}_{\mathrm{b}}$. The subtracted piece $\hat{G}_{\mathrm{s}}\left(\boldsymbol{p} ; z_{1}, z_{2}\right)=\hat{G}_{\mathrm{b}}\left(\boldsymbol{p} ; z_{1}+z_{2}\right)$ is the image term which ensures that the Dirichlet condition holds.

As mentioned in the caption of Fig. 3, we use open and crossed circles to indicate external points off and on the surface $\mathfrak{B}$, respectively, a broken line to represent $\hat{G}_{\mathrm{D}}$, and perpendicular strokes to mark $z$-derivatives $\partial_{z}$. Accordingly, Eq. (A1) can be depicted as

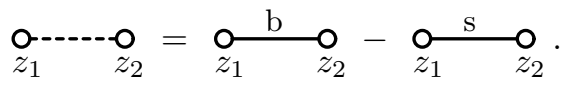

The Feynman graph expansion of the two-point function $\hat{G}_{\infty}^{(1,1)}\left(\boldsymbol{p} ; z_{1}\right)$ to two-loop order is shown in Fig. 3. The 
zero-loop term

$$
\stackrel{\mathrm{Z}}{1}_{1}^{-----1 \otimes}=\left(\hat{G}_{\mathrm{D}} \overleftarrow{\partial}_{n}\right)\left(\boldsymbol{p} ; z_{1}\right) \equiv \partial_{z_{2}} \hat{G}_{\mathrm{D}}\left(\boldsymbol{p} ; z_{1}, z_{2}=0\right)=e^{-\hat{\kappa}_{\boldsymbol{p}} z_{1}}
$$

follows directly from Eq. (A1).

In order to determine the one and two-loop terms, we amputate the external free legs of the graphs and compute the Laurent expansion of the resulting amputated graphs, considered as generalized functions (distributions) of the dimensionless variable $\mu z$ and $\mu z^{\prime}$ in the $\boldsymbol{p} z$ representation. ${ }^{28}$ The amputated legs play the role of test functions ${ }^{59}$ on which the latter distributions act. Once the Laurent expansions of the amputated graphs are known, those of the full graphs can be computed in a straightforward fashion by performing the remaining integrations over $z$ and $z^{\prime}$. For convenience, we will set $\mu=\sigma=1$ in most of the sequel. Whenever necessary, the dependences on $\mu$ and $\sigma$ can easily be reintroduced by dimensional arguments.

The one-loop term of $\hat{G}_{\infty}^{(1,1)}\left(\boldsymbol{p} ; z_{1}\right)$ reads

$$
z_{1}
$$

where the internal loop, $G_{\mathrm{D}}(\boldsymbol{x}, \boldsymbol{x})$, according to Eq. (13) is given by

$$
G_{\mathrm{D}}(\boldsymbol{x}, \boldsymbol{x})=-G_{b}(2 z \boldsymbol{n})=-F_{m, \epsilon} \frac{\Gamma(2-\epsilon) \sin (\epsilon \pi / 2)}{\epsilon \pi \dot{\circ}^{m / 4}} z^{\epsilon-2} .
$$

Considering $z^{\epsilon-2}$ as a generalized function, we can calculate its action on a test function $\varphi(z)$ and expand the result about $\epsilon=0$ to obtain 28,60

$$
\int_{0}^{\infty} d z z^{\epsilon-2} \varphi(z)=\left.\frac{1}{\epsilon} \frac{\partial \varphi(z)}{\partial z}\right|_{z=0}+\left(z_{+}^{-2}, \varphi(z)\right)+O(\epsilon)
$$

where $z_{+}^{-2}$ is a standard generalized function, defined as

$$
\left(z_{+}^{-2}, \varphi(z)\right) \equiv \int_{0}^{\infty} \frac{d z}{z^{2}}\left[\varphi(z)-\varphi(0)-\theta(1-z) z \varphi^{\prime}(0)\right] .
$$

Equations (A4)-(A5) yield

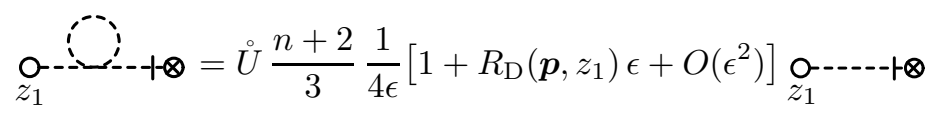

with

$$
\stackrel{\circ}{U}=\mu^{-\epsilon} \stackrel{\circ}{\mathrm{i}} \stackrel{\circ}{ }^{-m / 4} F_{m, \epsilon}
$$

and

$$
\begin{aligned}
& R_{\mathrm{D}}\left(\boldsymbol{p}, z_{1}\right) \equiv C_{E}-1+\left(z_{+}^{-2}, \hat{G}_{\mathrm{D}}\left(\boldsymbol{p} ; z_{1}, z\right)\left(\hat{G}_{\mathrm{D}} \overleftarrow{\partial}_{n}\right)(\boldsymbol{p} ; z)\right) / \mathrm{z}_{1}-\cdots--+-\otimes \\
& =\operatorname{Ei}\left(-2 \stackrel{\circ}{\kappa}_{\boldsymbol{p}} z_{1}\right) e^{2 \stackrel{\kappa}{\boldsymbol{p}}_{\boldsymbol{p}} z_{1}}-\ln (2 \stackrel{\kappa}{\kappa} \boldsymbol{p}),
\end{aligned}
$$

where $\operatorname{Ei}(x)$ is the exponential-integral function.

We next consider the two-loop diagram

$$
\begin{aligned}
& z_{1} \\
& \times \int_{0}^{\infty} d z^{\prime} G_{\mathrm{D}}\left(\boldsymbol{x}^{\prime}, \boldsymbol{x}^{\prime}\right) \int \frac{d^{d-1} \boldsymbol{p}^{\prime}}{(2 \pi)^{d-1}} \hat{G}_{\mathrm{D}}\left(\boldsymbol{p}^{\prime} ; z, z^{\prime}\right) \hat{G}_{\mathrm{D}}\left(-\boldsymbol{p}^{\prime} ; z, z^{\prime}\right) \text {. }
\end{aligned}
$$

Performing the $\boldsymbol{p}^{\prime}$ integration associated with the lower loop yields

$$
2 \times \vdots_{z}^{\prime} \vdots_{z}^{\prime}=\frac{1}{2} F_{m, \epsilon} \frac{\Gamma(1-\epsilon) \sin (\epsilon \pi / 2)}{\epsilon \pi \sigma^{m / 4}}\left\{\left|z-z^{\prime}\right|^{\epsilon-1}+\left(z+z^{\prime}\right)^{\epsilon-1}-2\left[\max \left(z, z^{\prime}\right)\right]^{\epsilon-1}\right\},
$$


where the multiplication by 2 on the left-hand side compensates the graph's line-symmetry factor of $1 / 2$, so that the result is precisely the integral over $\boldsymbol{p}^{\prime}$ (last integral) in Eq. (A12).

The subsequent $z^{\prime}$ integration in Eq. (A12) is straightforward, giving

$$
2 \int_{0}^{\infty} d z^{\prime} G_{\mathrm{D}}\left(\boldsymbol{x}^{\prime}, \boldsymbol{x}^{\prime}\right) \stackrel{c^{\bullet}}{z^{\prime}}=-\left[F_{m, \epsilon} \frac{\Gamma(2-\epsilon) \sin (\epsilon \pi / 2)}{\epsilon \pi \dot{\sigma}^{m / 4}}\right]^{2} \frac{1}{1-\epsilon}\left\{\frac{1}{1-\epsilon}+\frac{\Gamma(\epsilon) \Gamma(\epsilon-1)}{\Gamma(2 \epsilon-1)}\right\} z^{2 \epsilon-2} .
$$

Using Eqs. (A6) and (A7), one can easily compute the action of this distribution on the external lines. The Laurent expansion of the result becomes

$$
{ }_{z_{1}}
$$

The diagram

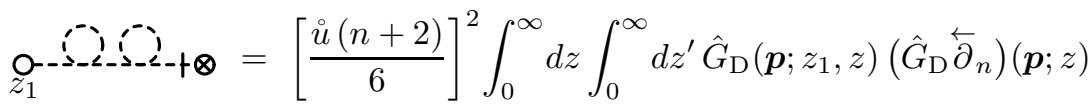

$$
\begin{aligned}
& \times \hat{G}_{\mathrm{D}}\left(\boldsymbol{p} ; z, z^{\prime}\right) G_{\mathrm{D}}(\boldsymbol{x}, \boldsymbol{x}) G_{\mathrm{D}}\left(\boldsymbol{x}^{\prime}, \boldsymbol{x}^{\prime}\right)
\end{aligned}
$$

is quite similar. Its resulting Laurent expansion reads

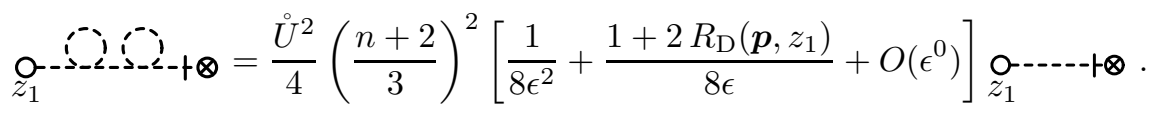

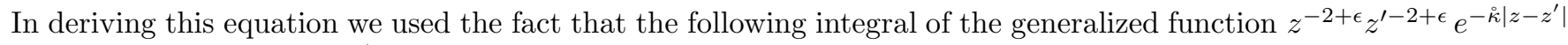
with a test function $\varphi\left(z, z^{\prime}\right)$ has the Laurent expansion

$$
\begin{aligned}
& \int_{0}^{\infty} d z \int_{0}^{\infty} d z^{\prime} \varphi\left(z, z^{\prime}\right) z^{-2+\epsilon} z^{\prime-2+\epsilon} e^{-\kappa\left|z-z^{\prime}\right|} \\
& =\frac{\left(\partial_{z} \partial_{z^{\prime}} F\right)(0,0)}{2 \epsilon^{2}}+\frac{1}{\epsilon}\left[\left(z_{+}^{\prime}{ }^{-2},\left(\partial_{z} F\right)\left(0, z^{\prime}\right)\right)+\frac{\left(\partial_{z}^{2} F\right)(0,0)-\left(\partial_{z^{\prime}}^{2} F\right)(0,0)}{4}\right]+O\left(\epsilon^{0}\right),
\end{aligned}
$$

where $F$ is defined by

$$
F\left(z, z^{\prime}\right) \equiv\left[\varphi\left(z, z^{\prime}\right)+\varphi\left(z^{\prime}, z\right)\right] e^{\stackrel{\wp}{\kappa}\left(z-z^{\prime}\right)} .
$$

In addition, Eqs. (A5) and (A6) were employed.

The computation of the diagram

On the position-space representation it can be written as

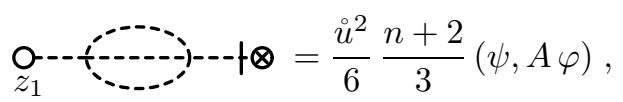

where $\mathcal{A}$ is an integral operator defined through

$$
(\psi, \mathcal{A} \varphi) \equiv \int_{\mathbb{R}_{+}^{d}} d^{d} x \int_{\mathbb{R}_{+}^{d}} d^{d} x^{\prime} \psi(\boldsymbol{x}) G_{\mathrm{D}}^{3}\left(\boldsymbol{x}, \boldsymbol{x}^{\prime}\right) \varphi\left(\boldsymbol{x}^{\prime}\right)
$$

with

$$
\psi(\boldsymbol{x}) \equiv G_{\mathrm{D}}\left(\boldsymbol{x}_{1}, \boldsymbol{x}\right), \quad \varphi\left(\boldsymbol{x}^{\prime}\right) \equiv\left(G_{\mathrm{D}} \overleftarrow{\partial}_{n}\right)\left(\boldsymbol{x}^{\prime}, \boldsymbol{r}_{2}\right)
$$

Since

$$
(\psi, \mathcal{A} \varphi)=(\psi, \mathcal{A} \varphi)=\{(\varphi+\psi, \mathcal{A}[\varphi+\psi])-(\varphi, \mathcal{A} \varphi)-(\psi, \mathcal{A} \psi)\} / 2,
$$

it is sufficient to calculate the diagonal element $(\varphi, \mathcal{A} \varphi)$ for a general test function $\varphi$. Upon substituting Eq. (A1) into Eq. (A21), one obtains

$$
(\varphi, \mathcal{A} \varphi)=I_{0}-3 I_{1}+3 I_{2}-I_{3}
$$


where $I_{j}$ is defined through

$$
I_{j} \equiv\left(\varphi, \mathcal{A}_{j} \varphi\right)=\int_{\mathbb{R}_{+}^{d}} d^{d} x \int_{\mathbb{R}_{+}^{d}} d^{d} x^{\prime} \varphi(\boldsymbol{x})\left[G_{\mathrm{b}}\left(\boldsymbol{x}-\boldsymbol{x}^{\prime}\right)\right]^{3-j}\left[G_{\mathrm{s}}\left(\boldsymbol{x}, \boldsymbol{x}^{\prime}\right)\right]^{j} \varphi\left(\boldsymbol{x}^{\prime}\right) .
$$

Let us calculate the terms on the right-hand side of Eq. (A24) one by one, starting with the "bulk" graph

$$
I_{0}=\int_{0}^{\infty} d z \int_{0}^{\infty} d z^{\prime} \int d^{d-1} r \int d^{d-1} r^{\prime} \varphi(\boldsymbol{r}, z) \varphi\left(\boldsymbol{r}^{\prime}, z^{\prime}\right) G_{\mathrm{b}}^{3}\left(\boldsymbol{r}-\boldsymbol{r}^{\prime},\left|z-z^{\prime}\right|\right) .
$$

Exploiting the scaling property of the free bulk propagator,

$$
G_{\mathrm{b}}(\boldsymbol{r} ; z) \equiv G_{\mathrm{b}}\left[\left(r_{\alpha}\right),\left(r_{\beta}\right) ; z\right]=|z|^{\epsilon-2} G_{\mathrm{b}}\left[\left(r_{\alpha}|z|^{-1 / 2}\right),\left(r_{\beta} /|z| ; 1\right]\right.
$$

and making the changes of variables $r_{\alpha}^{\prime} \rightarrow \rho_{\alpha} \equiv\left|z-z^{\prime}\right|^{-1 / 2}\left(r_{\alpha}^{\prime}-r_{\alpha}\right)$ and $r_{\beta}^{\prime} \rightarrow \rho_{\beta} \equiv\left|z-z^{\prime}\right|^{-1}\left(r_{\beta}^{\prime}-r_{\beta}\right)$, we can recast Eq. (A26) as

$$
I_{0}=\int d^{d-1} r \int_{0}^{\infty} d z \int_{0}^{\infty} d z^{\prime}\left|z-z^{\prime}\right|^{2 \epsilon-3} \varphi(\boldsymbol{r}, z) \int d^{d-1} \rho \varphi\left[\left(r_{\alpha}+\sqrt{\left|z-z^{\prime}\right|} \rho_{\alpha}\right),\left(r_{\beta}+\left|z-z^{\prime}\right| \rho_{\beta}\right), z^{\prime}\right] G_{\mathrm{b}}^{3}(\boldsymbol{\rho} ; 1) .
$$

This integral has a pole at $\epsilon=0$ caused by the ultraviolet divergence on the line $\left|z^{\prime}-z\right|=0$ which is due to the factor $\left|z-z^{\prime}\right|^{2 \epsilon-3}$. For our purposes here it is sufficient to compute just the singular part of the integral (A28). To this end, we expand its integrand in powers of $\sqrt{\left|z-z^{\prime}\right|} \rho_{\alpha}$ and $\left|z-z^{\prime}\right| \rho_{\beta}$. Subsequent termwise integration over $z^{\prime}$ then yields

$$
\begin{aligned}
I_{0}= & \frac{1}{\epsilon} \int d^{d-1} r \int_{0}^{\infty} d z \varphi(\boldsymbol{r}, z)\left\{\frac{f_{0}(1)}{2}\left[\frac{\partial^{2} \varphi(\boldsymbol{r}, z)}{\partial z^{2}}+\partial_{\beta} \partial_{\beta} \varphi(\boldsymbol{r}, z)\right]+\frac{f_{4}(1)}{8 m(m+2)}\left(\partial_{\alpha} \partial_{\alpha}\right)^{2} \varphi(\boldsymbol{r}, z)\right\} \\
& -\frac{f_{2}(1)}{4 \epsilon m} \int d^{d-1} r \varphi(\boldsymbol{r}, 0) \partial_{\alpha} \partial_{\alpha} \varphi(\boldsymbol{r}, 0)+O\left(\epsilon^{0}\right),
\end{aligned}
$$

where we have introduced

$$
f_{k}(t) \equiv\left\{\int d^{d^{*}-1} \rho\left(\rho_{\alpha^{\prime}} \rho_{\alpha^{\prime}}\right)^{k / 2} G_{\mathrm{b}}^{2}(\boldsymbol{\rho} ; 1) G_{\mathrm{b}}\left[\left(\rho_{\alpha} t^{1 / 2}\right),\left(\rho_{\beta} t\right) ; 1\right]\right\}_{\epsilon=0}, \quad k=0,2,4
$$

and utilized the identity

$$
\int_{0}^{\infty} d z \int_{0}^{\infty} d z^{\prime}\left|z-z^{\prime}\right|^{2 \epsilon-N} \chi(z) \chi\left(z^{\prime}\right)=\frac{1}{\epsilon(N-1) !} \int_{0}^{\infty} d z \chi(z) \frac{d^{N-1}}{d z^{N-1}} \chi(z)+O\left(\epsilon^{0}\right)
$$

valid for $N \in \mathbb{N}$ and test functions $\chi(z)$.

Next, we turn to the terms $I_{j}$ with $j>0$. The associated Feynman diagrams contain the image part $G_{\mathrm{s}}\left(\boldsymbol{x}, \boldsymbol{x}^{\prime}\right)=$ $G_{\mathrm{b}}\left(\boldsymbol{r}-\boldsymbol{r}^{\prime} ;\left|z+z^{\prime}\right|\right)$, which may induce additional ultraviolet singularities ${ }^{28}$ at $z=0$ and $z^{\prime}=0$. To compute

$$
I_{1}=\int_{\mathbb{R}_{+}^{d}} d^{d} x \int_{\mathbb{R}_{+}^{d}} d^{d} x^{\prime} \varphi(\boldsymbol{x}) G_{\mathrm{b}}^{2}\left(\boldsymbol{x}-\boldsymbol{x}^{\prime}\right) G_{\mathrm{s}}\left(\boldsymbol{x}, \boldsymbol{x}^{\prime}\right) \varphi\left(x^{\prime}\right),
$$

we transform from the integration variables $\left\{z, z^{\prime}, \boldsymbol{r}^{\prime}\right\}$ to new ones, $\{v, t, \boldsymbol{\rho}\}$, defined by

$$
z=v \frac{1+t}{2}, \quad z^{\prime}=v \frac{1-t}{2}, \quad r_{\alpha}^{\prime}=r_{\alpha}+\sqrt{v t} \rho_{\alpha}, \quad r_{\beta}^{\prime}=r_{\beta}+v t \rho_{\beta},
$$

and then use again the scaling property (A27) of the propagator to rewrite Eq. (A32) as

$$
I_{1}=\int d^{d-1} r \int_{0}^{\infty} d v v^{2 \epsilon-2} \int_{0}^{1} d t t^{\epsilon-1} \mathcal{G}(\boldsymbol{r}, v, t)
$$

with

$$
\begin{aligned}
\mathcal{G}(\boldsymbol{r}, v, t)= & \varphi[\boldsymbol{r}, v(1+t) / 2] \int d^{d-1} \rho \varphi\left[\left(r_{\alpha}+\sqrt{v t} \rho_{\alpha}\right),\left(r_{\beta}+v t \rho_{\beta}\right), v(1-t) / 2\right] \times \\
& \times G_{\mathrm{b}}^{2}(\boldsymbol{\rho} ; 1) G_{\mathrm{b}}\left[\left(t^{1 / 2} \rho_{\alpha}\right),\left(t \rho_{\beta}\right) ; 1\right] .
\end{aligned}
$$


Upon performing the integration over $t$ to obtain

$$
\int_{0}^{1} d t t^{\epsilon-1} \mathcal{G}(\boldsymbol{r}, v, t)=\frac{\mathcal{G}(\boldsymbol{r}, v, 0)}{\epsilon}+\int_{0}^{1} \frac{d t}{t}[\mathcal{G}(\boldsymbol{r}, v, t)-\mathcal{G}(\boldsymbol{r}, v, 0)]+O(\epsilon),
$$

we can do the integration over $v$ in Eq. (A34), using Eqs. (A6) and(A7). Straightforward calculations then yield the result

$$
\begin{aligned}
I_{1}= & \int d^{d-1} r\left\{\frac{f_{0}(0)}{2 \epsilon^{2}} \varphi(\boldsymbol{r}, 0) \varphi^{\prime}(\boldsymbol{r}, 0)+\frac{\varphi(\boldsymbol{r}, 0) \varphi^{\prime}(\boldsymbol{r}, 0)}{2 \epsilon} \int_{0}^{1} \frac{d t}{t}\left[f_{0}(t)-f_{0}(0)\right]\right. \\
& \left.+\frac{1}{4 m \epsilon} \varphi(\boldsymbol{r}, 0) \partial_{\alpha} \partial_{\alpha} \varphi(\boldsymbol{r}, 0) \int_{0}^{1} d t f_{2}(t)+\frac{f_{0}(0)}{\epsilon}\left(v_{+}^{-2}, \varphi^{2}(\boldsymbol{r}, v / 2)\right)\right\}+O\left(\epsilon^{0}\right) .
\end{aligned}
$$

The Laurent expansions of the integrals $I_{2}$ and $I_{3}$ can be worked out in a similar fashion. They read

$$
\begin{aligned}
I_{2}= & \int d^{d-1} r\left\{\frac{1}{2 \epsilon} \varphi(\boldsymbol{r}, 0) \varphi^{\prime}(\boldsymbol{r}, 0) \int_{1}^{\infty} d t f_{0}(t)\right. \\
& \left.+\frac{1}{4 m \epsilon} \varphi(\boldsymbol{r}, 0) \partial_{\alpha} \partial_{\alpha} \varphi(\boldsymbol{r}, 0) \int_{1}^{\infty} d t f_{2}(t)\right\}+O\left(\epsilon^{0}\right)
\end{aligned}
$$

and

$$
I_{3}=\int d^{d-1} r\left\{\frac{f_{0}(1)}{2 \epsilon} \varphi(\boldsymbol{r}, 0) \varphi^{\prime}(\boldsymbol{r}, 0)+\frac{f_{2}(1)}{4 m \epsilon} \varphi(\boldsymbol{r}, 0) \partial_{\alpha} \partial_{\alpha} \varphi(\boldsymbol{r}, 0)\right\}+O\left(\epsilon^{0}\right) .
$$

Equations (A29), (A37), (A38) and (A39) give us the diagonal elements $\left(\varphi, \mathcal{A}_{j} \varphi\right)$ for a general test function $\varphi$. Utilizing these results in conjunction with Eq. (A23), one can readily determine the required off-diagonal elements $\left(\psi, \mathcal{A}_{j} \varphi\right)$ corresponding to the special choices (A22) of $\psi(\boldsymbol{x})$ and $\varphi(\boldsymbol{x})$. The terms involving $f_{2}$ do not contribute to the pole terms because of the Dirichlet boundary condition satisfied by the test function $\psi$ from Eq. (A22). This entails that there are no such contributions from the diagonal elements $\left(\psi, \mathcal{A}_{j} \psi\right)$, and that there analogs from the difference $\left(\psi+\phi, \mathcal{A}_{j}[\psi+\phi]\right)-\left(\phi, \mathcal{A}_{j} \phi\right)$ cancel.

The coefficients $f_{0}(1)$ and $f_{4}(1)$ can be expressed in terms of the single integrals $j_{\phi}(m)$ and $j_{\sigma}(m)$ defined by Eqs. (47) and (48) as

$$
f_{0}(1)=F_{m, 0}^{2} \stackrel{\circ}{\sigma}^{-m / 2} \frac{j_{\phi}(m)}{8-m}
$$

and

$$
f_{4}(1)=F_{m, 0}^{2} \stackrel{\circ}{\sigma}^{1-m / 2} \frac{j_{\sigma}(m)}{2}
$$

The two integrals involving $f_{0}(t)$,

$$
\mathcal{I}_{1} \equiv \int_{0}^{1} \frac{d t}{t}\left(f_{0}(t)-f_{0}(0)\right)
$$

and

$$
\mathcal{I}_{2} \equiv \int_{1}^{\infty} d t f_{0}(t)=\int_{0}^{1} \frac{d t}{t^{2}} f_{0}(1 / t)
$$

both yield contributions $\sim \epsilon^{-1}$. Let us rewrite them in terms of the integrals $J_{u}(m)$ and $j_{1}(m)$ defined by Eqs. (43), (44), and (76).

To this end, we rewrite $\mathcal{I}_{1}$ as

$$
\mathcal{I}_{1}=\lim _{\delta \rightarrow 0+}\left(B_{\delta}+f_{0}(0) \ln \delta\right), \quad B_{\delta} \equiv \int_{\delta}^{1} \frac{d t}{t} f_{0}(t)
$$

Using the scaling form (10) of the free bulk propagator and performing the angular integrations, one obtains

$$
\begin{aligned}
\frac{B_{\delta} \stackrel{\circ}{\sigma}^{m / 2}}{S_{m} S_{3-m / 2}} & =\int_{\delta}^{1} d t \int_{0}^{\infty} d r r^{2-m / 2} \int_{0}^{\infty} d w\left(t^{2}+r^{2}\right)^{m / 4} \frac{\Phi_{m, d^{*}}^{2}\left(w^{1 / 4}\right) \Phi_{m, d^{*}}\left[(y(t, r) w)^{1 / 4}\right]}{4 w^{1-m / 4}\left(1+r^{2}\right)\left(t^{2}+r^{2}\right)^{2}} \\
& =\int_{0}^{\infty} d w \frac{\Phi_{m, d^{*}}^{2}\left(w^{1 / 4}\right)}{8 w^{1-m / 4}} \int_{0}^{\infty} d r \frac{r^{2-m / 2}}{\left(1+r^{2}\right)^{2-m / 4}} \int_{y(\delta, r)}^{1} d y \frac{\Phi_{m, d^{*}}\left[(y w)^{1 / 4}\right]}{y^{2-m / 4} \sqrt{y\left(1+r^{2}\right)-r^{2}}}
\end{aligned}
$$


where

$$
y(t, r) \equiv \frac{t^{2}+r^{2}}{1+r^{2}}
$$

is the new integration variable to which we transformed to get the second line of Eq. (A45). To extract the singularity of the integral for small $\delta$, we subtract and add $\Phi_{m, d^{*}}(0)$ in the numerator of the $y$ integral. In the part of the integral produced by the term $\Phi_{m, d^{*}}\left[(y w)^{1 / 4}\right]-\Phi_{m, d^{*}}(0)$, we can safely replace the lower integration limit by its $\delta \rightarrow 0$ limit $y(0, r)$. In the double integral $\int_{0}^{\infty} d r \int_{y(0, r)}^{\infty} d y$ we reverse the order of integrations whereby it becomes $\int_{0}^{1} d y \int_{0}^{r} y d r$ with $r_{y}=\sqrt{y /(1-y)}$. Upon changing from $r$ to the integration variable $x=r / r_{y}$, we can perform the $x$ integration. In the $w$ integral of the resulting expression, one easily recognizes the scaling function $\Theta_{m}\left(w^{1 / 4}\right)$ given in Eq. (45) upon recalling Eq. (D.1) of Ref. 15. The result of the remaining $w$ integration therefore is proportional to the integral $j_{u}(m)$ whose definition is recalled in Eq. (44).

The part of the integral associated with the constant $\Phi_{m, d^{*}}(0)$ in the numerator of Eq. (A45) yields terms that behave as $\sim \ln \delta$ and approach a finite limit as $\delta \rightarrow 0$, respectively. They can be computed in a straightforward fashion. Upon gathering all contributions to $B_{\delta}$, substituting them into Eq. (A44), and taking into account that $f_{0}(0)=\stackrel{\circ}{\sigma}^{-m / 2} F_{m, 0}^{2}$, we can perform the required limit $\delta \rightarrow 0$. The result is

$$
\mathcal{I}_{1} / F_{m, 0}^{2} \stackrel{\circ}{\sigma}^{-m / 2}=\int_{0}^{1} \frac{d t}{t}\left[\frac{f_{0}(t)}{f_{0}(0)}-1\right]=J_{u}(m)-1-\ln 2 .
$$

The integral $\mathcal{I}_{2}$ can be transformed in a similar manner to show that it can be expressed in terms of the integral $j_{1}(m)$ introduced in Eq. (76) as

$$
\mathcal{I}_{2} / F_{m, 0}^{2} \stackrel{\circ}{\sigma}^{-m / 2}=\int_{1}^{\infty} d t \frac{f_{0}(t)}{f_{0}(0)}=j_{1}(m) .
$$

Combining the above results finally yields

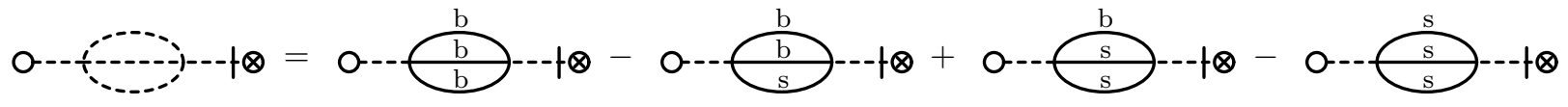

with

$$
\begin{aligned}
& \stackrel{\mathrm{b}}{\mathrm{b}} \mathrm{z}_{1}--+\mathrm{b} \otimes=\frac{n+2}{3} \stackrel{\circ}{U}^{2}\left\{\frac{\stackrel{\circ}{\sigma} z_{1}\left(p_{\alpha} p_{\alpha}\right)^{2}}{2 \stackrel{\circ}{\kappa}_{\boldsymbol{p}} \epsilon}\left[\frac{j_{\phi}(m)}{12(8-m)}+\frac{j_{\sigma}(m)}{96 m(m+2)}\right]\right. \\
& \left.-\frac{j_{\phi}(m)}{24 \epsilon(8-m)}+O\left(\epsilon^{0}\right)\right\} \underset{z_{1}}{\mathrm{O}-\cdots---r \otimes}
\end{aligned}
$$

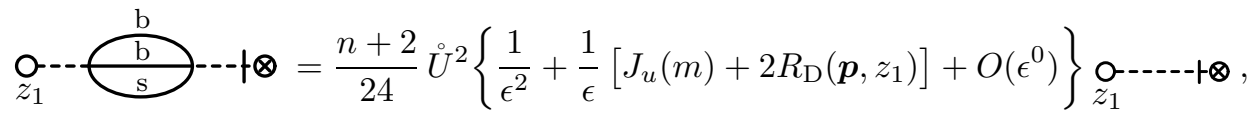

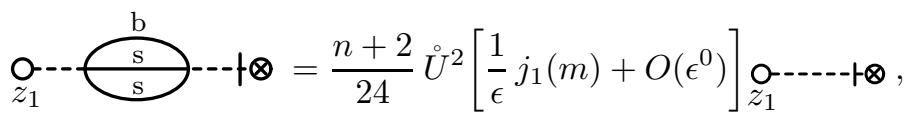

and

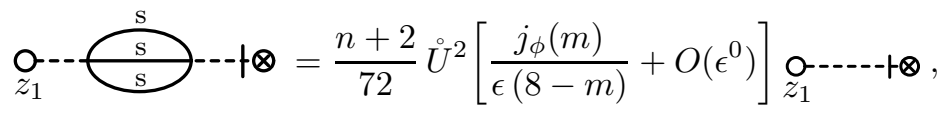

where it should be noticed that the line-symmetry factors of the graphs on the right-hand side of Eq. (A49) are $1 / 2$ (second and third graph) and 1/3! (all others), depending on whether they have two or three equivalent lines.

We can now substitute the above results into Eq. $(73)$ for $G_{\infty}^{(0,2)}(\boldsymbol{p} ; z)$ and express the bare variables $\stackrel{\circ}{U}^{\circ}$ and $\stackrel{\circ}{\sigma}$ in terms of renormalized ones using the expressions for the bulk renormalization factors $Z_{\phi}$, $Z_{\sigma}$, and $Z_{u}$ given in Eqs. (40), (41), and (48) of Ref. 15. Upon determining the renormalization factor $Z_{1, \infty}$ such that the dimensional poles are minimally subtracted, one easily arrives at the series expansion (74). 


\section{APPENDIX B: RENORMALIZATION OF $\hat{G}_{\infty}^{(0,2)}(\boldsymbol{p})$ TO TWO-LOOP ORDER}

In this appendix we consider the renormalization of the two-point function $G_{\infty}^{(0,2)}(\boldsymbol{p})$ for $\rho=\tau=0$, which requires the additive surface counterterm $\propto C_{2}(u, \epsilon)$ according to Eq. (73). At two-loop order the sole contribution to this counterterm comes from the diagram $\otimes-+\bullet:-\boldsymbol{-} \otimes$. To determine its pole terms one can proceed as in Appendix A, choosing the test functions as

$$
\psi(\boldsymbol{x}) \equiv\left(G_{\mathrm{D}} \overleftarrow{\partial}_{n}\right)\left(\boldsymbol{x}, \boldsymbol{r}_{2}\right), \quad \varphi\left(\boldsymbol{x}^{\prime}\right) \equiv\left(G_{\mathrm{D}} \overleftarrow{\partial}_{n}\right)\left(\boldsymbol{x}^{\prime}, \boldsymbol{r}_{2}\right)
$$

A straightforward calculation shows that the graph's pole terms in question - i. e., those with no dependence on $p_{\beta}$ and a $p_{\alpha}$ dependence of the form $\epsilon^{-1} p_{\alpha} p_{\alpha}$ - can be written as

$$
\frac{\stackrel{\circ}{U}^{2}}{8 m \epsilon} \frac{n+2}{9} \frac{\stackrel{\circ}{ }^{1 / 2} p_{\alpha} p_{\alpha}}{F_{m, 0}^{2}}\left[2 f_{2}(1)+3 \int_{0}^{1} d t f_{2}(t)-3 \int_{1}^{\infty} d t f_{2}(t)\right]_{\sigma=1},
$$

where $\stackrel{\circ}{U}$ and $f_{2}(t)$ are defined through Eqs. (A9) and (A30), respectively. Upon introducing the coefficient

$$
b_{m} \equiv \frac{S_{m} S_{3-\frac{m}{2}}}{4 m F_{m, 0}^{2}}=\frac{2^{5+m} \pi^{(22+3 m) / 4} \Gamma\left(1+\frac{m}{2}\right)}{\Gamma\left(\frac{3}{2}-\frac{m}{4}\right) \Gamma^{2}\left(1+\frac{m}{4}\right)}
$$

the two integrals can be written as

$$
\frac{F_{m, 0}^{-2}}{8 m} \int_{1}^{\infty} d t f_{2}(t)=b_{m} I^{(1)}(m),\left.\quad \frac{F_{m, 0}^{-2}}{8 m} \int_{0}^{1} d t f_{2}(t)\right|_{\sigma=1}=b_{m} I^{(2)}(m)
$$

with

$$
I^{(1)}(m)=\int_{0}^{\infty} d v v^{m+1} \Phi_{m, d^{*}}(v) \int_{0}^{1} d y y^{m+1} \Phi_{m, d^{*}}^{2}(v y) \Psi_{m}^{(1)}\left(y^{4}\right)
$$

and

$$
I^{(2)}(m)=\int_{0}^{\infty} d v v^{m+1} \Phi_{m, d^{*}}(v) \int_{1}^{\infty} d y y^{m+1} \Phi_{m, d^{*}}^{2}(v y) \Psi_{m}^{(2)}\left(y^{4}\right)
$$

where

$$
\Psi_{m}^{(1)}(y) \equiv \frac{1}{2 \sqrt{y}}\left(\frac{y}{1-y}\right)^{(6-m) / 4} \int_{0}^{1} d t \frac{t^{(2-m) / 4}}{\sqrt{1-t}}\left(1+\frac{y t}{1-y}\right)^{(m-6) / 4}
$$

and

$$
\Psi_{m}^{(2)}(y) \equiv \frac{1}{2 \sqrt{y}}\left(\frac{y}{y-1}\right)^{(6-m) / 4} \int_{0}^{\infty} d t \frac{t^{(2-m) / 4}}{\sqrt{1+t}}\left(1+\frac{y t}{y-1}\right)^{(m-6) / 4}
$$

The latter functions are expressible in terms of hypergeometric functions as

$$
\Psi_{m}^{(1)}(y)=\frac{\sqrt{\pi} y^{1-m / 4}(1-y)^{(m-6) / 4}}{2 \Gamma(2-m / 4)} \Gamma\left(\frac{3}{2}-\frac{m}{4}\right){ }_{2} F_{1}\left(\frac{3}{2}-\frac{m}{4}, \frac{3}{2}-\frac{m}{4} ; 2-\frac{m}{4} ; \frac{y}{y-1}\right)
$$

and

$$
\begin{aligned}
\Psi_{m}^{(2)}(y)= & \frac{\sqrt{\pi} \Gamma(1-m / 4)}{\left.2(y-1)^{1 / 2} \Gamma[(6-m) / 4)\right]}{ }_{2} F_{1}\left(\frac{1}{2}, \frac{1}{2} ; \frac{m}{4}, \frac{y}{y-1}\right) \\
& +\frac{y^{1-m / 4}(y-1)^{(m-6) / 4}}{2 \sqrt{\pi}} \Gamma\left(\frac{3}{2}-\frac{m}{4}\right) \Gamma\left(\frac{m}{4}-1\right) \times \\
& \times{ }_{2} F_{1}\left(\frac{3}{2}-\frac{m}{4}, \frac{3}{2}-\frac{m}{4} ; 2-\frac{m}{4} ; \frac{y}{y-1}\right) .
\end{aligned}
$$


From the above results, Eq. (75) for $C_{2}(u, \epsilon)$ follows at once.

As a consistency check we have explicitly verified to two-loop order that all poles of $G_{\infty}^{(0,2)}(\boldsymbol{p} ; \sigma=\tau=0)$ get canceled in the renormalized function $G_{\infty, \operatorname{ren}}^{(0,2)}(\boldsymbol{p} ; \sigma=\tau=0)$ for our choice of bulk and surface renormalization functions. For interested readers wishing to check this, we here give the Laurent expansions of the corresponding Feynman diagrams to the required orders in $\epsilon$ and $\stackrel{\circ}{U}$. They read

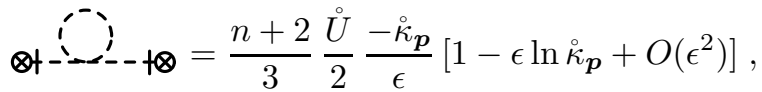

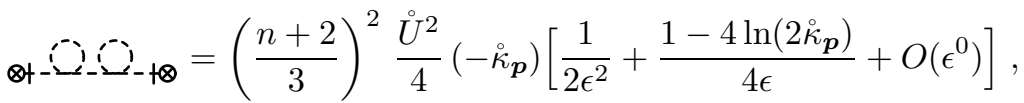

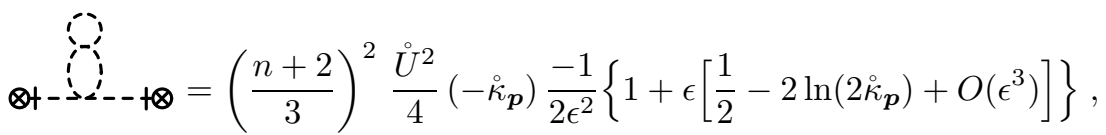

and

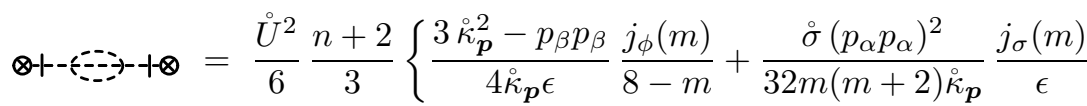

$$
\begin{aligned}
& +6 \frac{\sqrt{\circ} p_{\alpha} p_{\alpha}}{\epsilon} b_{m}\left[\frac{2}{3} \frac{J_{2,3}(m)}{6-m}-I^{(1)}(m)+I^{(2)}(m)\right] \\
& \left.+\frac{3 \stackrel{\circ}{\kappa}_{\boldsymbol{p}}}{2 \epsilon}\left[\frac{1}{\epsilon}+J_{u}(m)-j_{1}(m)-2 \ln \left(2 \stackrel{\circ}{\kappa}_{\boldsymbol{p}}\right)\right]+O\left(\epsilon^{0}\right)\right\} \text {, }
\end{aligned}
$$

where $J_{2,3}(m)$ denotes a particular one of the integrals $(49)$.

\section{APPENDIX C: ONE-LOOP CALCULATION FOR GENERAL VALUES OF $\lambda$}

In this appendix we describe the calculation of the renormalization functions $Z_{1}(u, \lambda, \epsilon), Z_{c}(u, \lambda, \epsilon)$, and $P_{\lambda}(u, \lambda, \epsilon)$, defined by Eqs. (18), to one-loop order. For this purpose, it is sufficient to consider the cumulants $\hat{G}^{(1,1)}\left(\boldsymbol{p} ; z_{1}\right)$ and $\partial \hat{G}^{(2,0)}\left(\boldsymbol{p} ; z_{1}\right) / \partial \stackrel{\circ}{\mathrm{c}}$ for $\stackrel{\circ}{c}=0$ and generic $\stackrel{\circ}{\lambda}>0$. Let us indicate that we have set $\stackrel{\circ}{c}=0$ by a subscript zero. The free propagator (7) in this case reduces to

$$
\hat{G}_{0}\left(\boldsymbol{p} ; z_{1}, z_{2}\right)=\frac{1}{2 \stackrel{\circ}{\kappa}_{\boldsymbol{p}}}\left[e^{-\stackrel{\circ}{\kappa}_{\boldsymbol{p}}\left|z_{1}-z_{2}\right|}+\frac{\stackrel{\circ}{\kappa} \boldsymbol{p}^{\circ} \stackrel{\circ}{\lambda} p_{\alpha} p_{\alpha}}{\stackrel{\circ}{\kappa} \boldsymbol{p}+\stackrel{\circ}{\lambda} p_{\alpha} p_{\alpha}} e^{-\stackrel{\circ}{\kappa}_{\boldsymbol{p}}\left(z_{1}+z_{2}\right)}\right]
$$

Consider first $\hat{G}_{0}^{(1,1)}\left(\boldsymbol{p} ; z_{1}\right)$. Up to one-loop order, we have

$$
\hat{G}_{0}^{(1,1)}\left(\boldsymbol{p} ; z_{1}\right)=\frac{e^{-\stackrel{\circ}{\kappa}_{p} z_{1}}}{\stackrel{\circ}{\kappa}_{\boldsymbol{p}}+\stackrel{\circ}{\lambda} p_{\alpha} p_{\alpha}}-\frac{\stackrel{\circ}{2}}{2} \frac{n+2}{3} \int_{0}^{\infty} d z \hat{G}_{0}\left(\boldsymbol{p} ; z_{1}, z\right) \hat{G}_{0}(\boldsymbol{p} ; z, 0) G_{0}(\boldsymbol{x}, \boldsymbol{x})+O\left(\stackrel{\circ}{ }^{2}\right)
$$

with

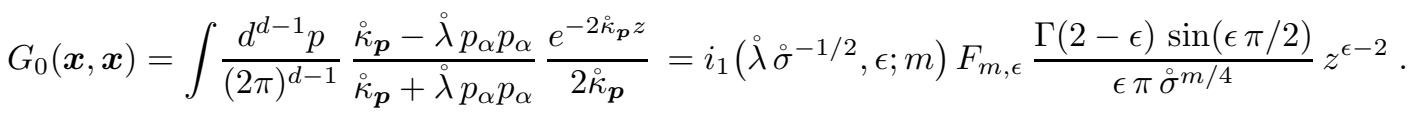

Here $i_{1}(\lambda, \epsilon ; m)$ is defined by

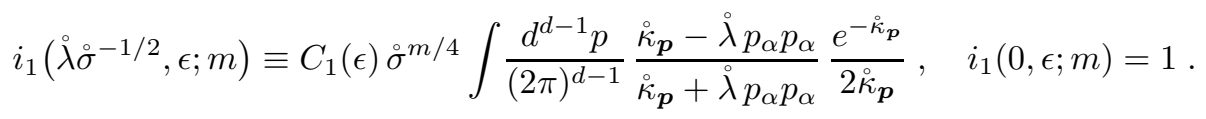

where the normalization constant $C_{1}$ is fixed by the specified value of $i_{1}(0, \epsilon ; m)$. Thus the $\dot{\lambda}$ dependence of the generalized function $G_{0}(\boldsymbol{x}, \boldsymbol{x})$ is entirely contained in the prefactor $i_{1}\left(\lambda \stackrel{\circ}{\sigma^{-1} / 2}, \epsilon ; m\right)$. 
After performing the angular integrations in Eq. (C4), we transform from the radial integration variables $P \equiv\left(p_{\beta} p_{\beta}\right)^{1 / 2}$ and $k=\left(\stackrel{\circ}{\sigma} p_{\alpha} p_{\alpha}\right)^{1 / 2}$ to $\kappa \equiv \stackrel{\circ}{\kappa}_{\boldsymbol{p}}$ and $k$, using $\int_{0}^{\infty} d k \int_{0}^{\infty} d p g(k, p)=\int_{0}^{\infty} d \kappa \int_{0}^{\sqrt{\kappa}} d k \kappa\left(\kappa^{2}-\right.$ $\left.k^{4}\right)^{-1 / 2} g\left(k, \sqrt{\kappa^{2}-k^{4}}\right)$. The $k$ integration then can be performed, and one easily convinces oneself that $i_{1}(\lambda, \epsilon ; m)$ can be written in the form (24).

We can now employ Eq. (A6) to perform the $z$ integration in Eq. (C2). This yields

$$
\begin{aligned}
& \hat{G}_{0}^{(1,1)}\left(\boldsymbol{p} ; z_{1}\right)=\left\{1+\stackrel{\circ}{\frac{n+2}{12}} i_{1}\left(\stackrel{\circ}{\lambda} \stackrel{\circ}{\sigma}^{-1 / 2} ; m\right)\left[\frac{1}{\epsilon}\left(1-\frac{2 \stackrel{\circ}{\lambda} p_{\alpha} p_{\alpha}}{\stackrel{\circ}{\kappa}_{\boldsymbol{p}}+\stackrel{\circ}{\lambda} p_{\alpha} p_{\alpha}}\right)+O\left(\epsilon^{0}\right)\right]\right\} \frac{e^{-\stackrel{\circ}{\kappa}_{\boldsymbol{p}} z_{1}}}{\stackrel{\circ}{\kappa} \boldsymbol{p}+\stackrel{\circ}{\lambda} p_{\alpha} p_{\alpha}} \\
& +O\left(\dot{u}^{2}\right) \text {. }
\end{aligned}
$$

To determine $Z_{1}$, we can set $p_{\alpha}=0$ and determine the $O(u)$ term of $Z_{1}(u, \lambda, \epsilon)$ from the condition that the pole of $\hat{G}_{0}^{(1,1)}\left(\boldsymbol{p} ; z_{1}\right)$ cancels in $\hat{G}_{0 \text {,ren }}^{(1,1)}\left(\boldsymbol{p} ; z_{1}\right)$. This yields the result $(21)$. For $p_{\alpha} \neq 0$, the pole implied by the first term of Eq. (C5) in parentheses cancels upon multiplication of $Z_{1}^{-1 / 2}$. Requiring that the remaining one implied by the

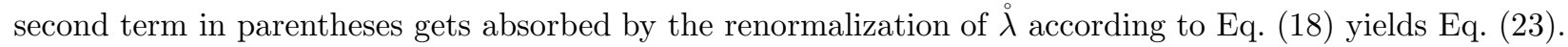

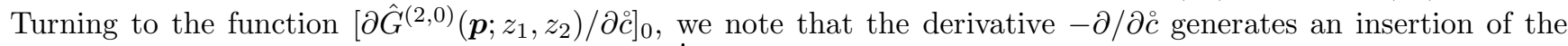

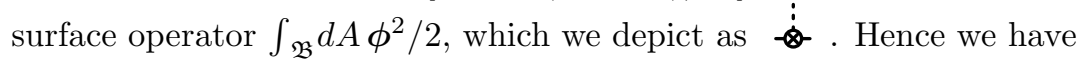

$$
\begin{aligned}
& \left.\frac{-\partial}{\partial \stackrel{\circ}{G}} \hat{G}^{(2,0)}\left(\boldsymbol{p} ; z_{1}, z_{2}\right)\right|_{\grave{c}=0}
\end{aligned}
$$

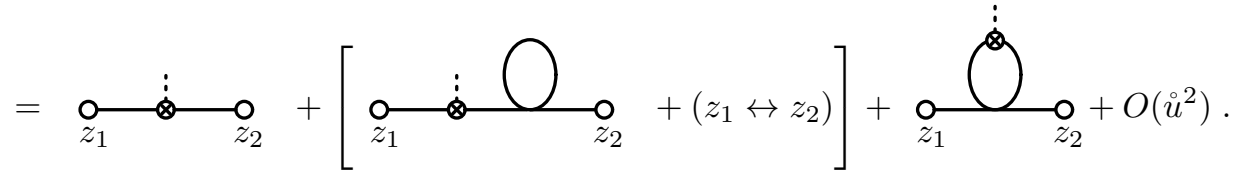

The Feynman integral of the graph inside the brackets is nothing but $\hat{G}\left(\boldsymbol{p} ; z_{1}, 0\right)$ times the term $\propto \stackrel{\circ}{u}$ of Eq. (C2), with the right external point taken at $z_{2}$. The remaining last graph's loop involves the integral

$$
\int \frac{d^{d-1} p}{(2 \pi)^{d-1}} \frac{e^{-2 \stackrel{\circ}{p}_{p} z}}{\left(\stackrel{\circ}{\kappa}_{\boldsymbol{p}}+\stackrel{\circ}{\lambda} p_{\alpha} p_{\alpha}\right)^{2}}=F_{m, \epsilon} \frac{4 \Gamma(1-\epsilon) \sin (\epsilon \pi / 2)}{\epsilon \pi \stackrel{\circ}{\sigma}^{m / 4}} i_{2}\left(\stackrel{\circ}{\lambda} \sigma^{-1 / 2} ; m\right) z^{\epsilon-1},
$$

where $i_{2}$ is the counterpart of $i_{1}$ defined by

$$
i_{2}\left(\stackrel{\lambda}{\circ}^{-1 / 2}, \epsilon ; m\right) \equiv C_{2}(\epsilon) \stackrel{\circ}{\sigma}^{m / 4} \int \frac{d^{d-1} p}{(2 \pi)^{d-1}} \frac{e^{-\stackrel{\kappa}{\kappa}_{p}}}{\left(\stackrel{\circ}{\kappa} \boldsymbol{p}+\stackrel{\circ}{\lambda} p_{\alpha} p_{\alpha}\right)^{2}}, \quad i_{2}(0, \epsilon ; m)=1 .
$$

Proceeding as in the case of $i_{1}$, one arrives at the form (25).

A combination of the above results yields the Laurent expansion

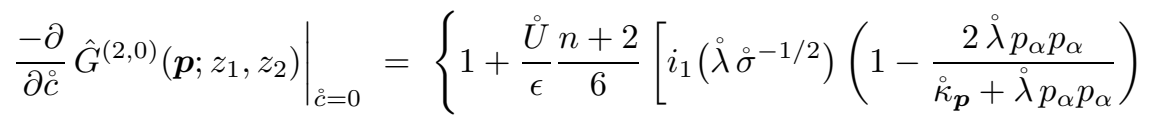

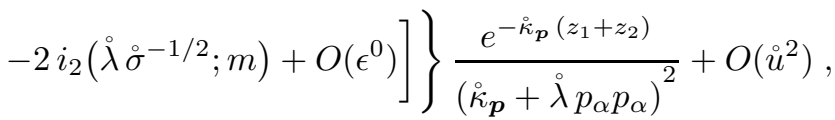

from which $Z_{c}$ can be determined in a straightforward fashion by requiring that the poles of the function (C9) are minimally absorbed through $Z_{c}$ so that the renormalized quantity $\left[\partial \hat{G}_{\text {ren }}^{(2,0)}\left(\boldsymbol{p} ; z_{1}, z_{2}\right) / \partial c\right]_{c=0}=$ $Z_{c} Z_{\phi}^{-1}\left[\partial \hat{G}^{(2,0)}\left(\boldsymbol{p} ; z_{1}, z_{2}\right) / \partial{ }^{\circ}\right]_{0}=\left[\partial \hat{G}_{\mathrm{ren}}^{(2,0)}\left(\boldsymbol{p} ; z_{1}, z_{2}\right) / \partial c\right]_{0}$ becomes uv finite. The result is given in Eq. (22).

Using Mathematica, ${ }^{61}$ the integrals $i_{1}(\lambda ; m) \equiv i_{1}(\lambda, \epsilon=0 ; m)$ and $i_{2}(\lambda ; m) \equiv i_{2}(\lambda, \epsilon=0 ; m)$ introduced in Eqs. (24) and (25) can be computed for $0<m<6$ and expressed in terms of hypergeometric functions. One obtains

$$
i_{1}(\lambda ; m)=\frac{m}{6} \lambda^{2}{ }_{2} F_{1}\left(1,1+\frac{m}{4} ; \frac{5}{2} ; \lambda^{2}\right)+{ }_{2} F_{1}\left(1, \frac{m}{4} ; \frac{3}{2} ; \lambda^{2}\right)-\frac{2 \pi\left[1-\left(1-\lambda^{2}\right)^{(2-m) / 4}\right]}{\lambda \cos (m \pi / 4) B[m / 4,(6-m) / 4]}
$$

and

$$
i_{2}(\lambda ; m)={ }_{2} F_{1}\left(1, \frac{m}{4} ; \frac{1}{2} ; \lambda^{2}\right)-\frac{\pi \lambda\left(1-\lambda^{2}\right)^{-(2+m) / 4}}{\cos (m \pi / 4) B[(2-m) / 4, m / 4]}
$$


For $m \rightarrow 0, m=2, m=4$, and $m \rightarrow 6$, these expressions simplify to

$$
\begin{aligned}
i_{1}(0+, m) & =1, \\
i_{1}(\lambda ; 2) & =-1+\frac{2}{\lambda} \ln (1+\lambda), \\
i_{1}(\lambda ; 4) & =\frac{\pi-\lambda}{\lambda}-\frac{2 \arccos \lambda}{\lambda \sqrt{1-\lambda^{2}}}, \\
i_{1}(\lambda ; 6-) & =\frac{1-\lambda}{1+\lambda},
\end{aligned}
$$

and

$$
\begin{aligned}
i_{2}(0+; m) & =1 \\
i_{2}(\lambda ; 2) & =\frac{1}{1+\lambda}, \\
i_{2}(\lambda ; 4) & =\frac{1-\lambda \arccos \lambda}{1-\lambda^{2}}, \\
i_{2}(\lambda ; 6-) & =\frac{1}{(1+\lambda)^{2}},
\end{aligned}
$$

respectively.

\section{APPENDIX D: CALCULATION OF $P_{\lambda}^{(2,-1)}(0)$}

In this appendix we wish to determine the renormalization (18) of $\stackrel{\lambda}{\text { to order }} u^{2}$ for $\lambda=0$ and compute the residuum $P_{\lambda}^{(2,-1)}(0)$ introduced by Eq. $(20)$.

To this end, we consider the function $\hat{G}_{0}^{(1,1)}\left(\boldsymbol{p} ; z_{1}\right) \equiv \hat{G}_{\dot{c}=0}^{(1,1)}\left(\boldsymbol{p} ; z_{1}\right)$ for $c=\stackrel{\circ}{c}=0$ and $\lambda=0$. As claimed, the bare variable $\lambda$ does not vanish at order $u^{2}$ if $\lambda=0$. Hence we must keep a nonzero $\dot{\lambda}$ in our calculation. Up to two-loop order, the Feynman graph expansion of $\hat{G}_{0}^{(1,1)}\left(\boldsymbol{p} ; z_{1}\right)$ reads

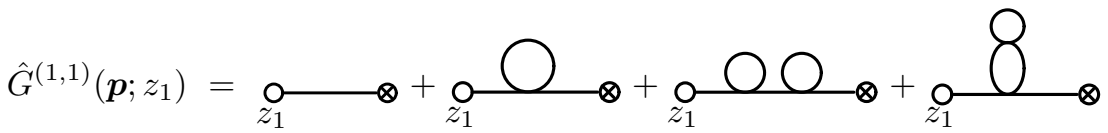

$$
\begin{aligned}
& +\bigcirc z_{1} \circlearrowright-O\left(\dot{u}^{3}\right) \text {, }
\end{aligned}
$$

where $\underset{z}{0} \mathrm{z}_{z^{\prime}}$, here represents the free propagator $(7)$ for $\stackrel{\circ}{c}=\stackrel{\circ}{\tau}=0$. As before, the crossed circle denotes a point on the surface. Thus the first graph simply becomes

$$
\underset{z_{1}}{\mathrm{\longrightarrow}} \otimes \frac{\exp \left(-\stackrel{\circ}{\kappa}_{\boldsymbol{p}} z\right)}{\stackrel{\circ}{\kappa} \boldsymbol{p}_{\boldsymbol{\lambda}} p_{\alpha} p_{\alpha}} .
$$

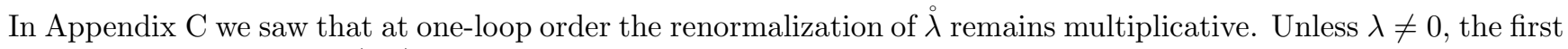
two two-loop graphs in Eq. (D1) obviously cannot produce primitive momentum dependent uv singularities of a form corresponding to the renormalization of the boundary operator $\sum_{\alpha}\left(\partial_{\alpha} \phi\right)^{2}$ because the (tadpole) graphs one obtains through amputation of the external lines are independent of the momentum $\boldsymbol{p}$. This momentum independence holds, of course, also if $\lambda \neq 0$. In this case, uv singularities requiring a multiplicative renormalization of $\lambda^{2}$ are produced by the pole $\sim \delta^{\prime}(z)$ of the amputated one-loop tadpole graph. As we explicitly showed in Appendix C, this follows quite simply by computing the action of this distribution on the external legs. This does not happen for $\lambda=0$ as long as $\lambda$ may be set to zero in the free propagator so that it obeys Neumann boundary conditions. The upshot of these considerations is that the first graph contributing to the (non-multiplicative) renormalization of $\dot{\lambda}$ is the right-most two-loop graph in Eq. (D1), involving the momentum dependent subgraph

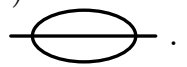

Since we know that $\stackrel{\lambda}{\lambda}=O\left(u^{2}\right)$ if $\lambda=0$, we can set $\stackrel{\lambda}{\lambda}=0$ in all but the first graph of Eq. (D1). Thus the lines of all those graphs correspond to the free Neumann propagator, which in analogy to Eq. (A1) can be written as 
$\hat{G}_{\mathrm{N}}=\hat{G}_{\mathrm{b}}+\hat{G}_{\mathrm{s}}$. Using this decomposition and the graphical conventions introduced in Eqs. (A2) and (A49), we may split the two-loop graph in question as

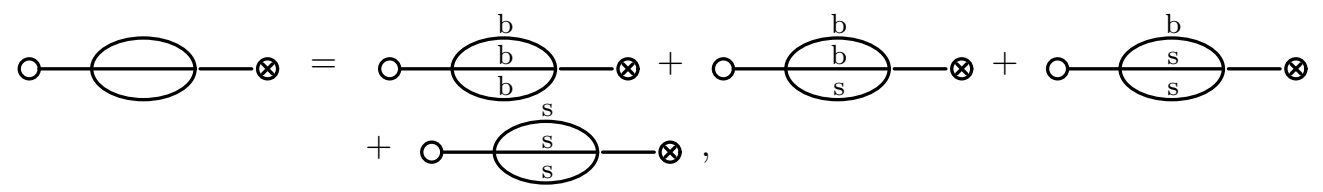

Each term on the right-hand side can be computed in the manner described in Appendix D. One chooses the functions $\phi$ and $\psi$ as $^{59}$

$$
\psi(\boldsymbol{x})=G_{\mathrm{N}}\left(\boldsymbol{x}_{1}, \boldsymbol{x}\right), \quad \varphi\left(\boldsymbol{x}^{\prime}\right)=\left.G_{\mathrm{N}}\left(\boldsymbol{x}^{\prime}, \boldsymbol{x}_{2}\right)\right|_{z_{2}=0},
$$

utilizes Eqs. (A23)-(A25), (A29), and (A37)-(A39), and finally computes the Fourier transform $\int d^{d-1} r_{12} \ldots e^{i \boldsymbol{p} \cdot \boldsymbol{r}_{12}}$.

Only those parts of the quantities $I_{j}$ that are proportional to $\int d^{d-1} r \varphi(\boldsymbol{r}, 0) \partial_{\alpha} \partial_{\alpha} \varphi(\boldsymbol{r}, 0)$ contribute to the renormalization of $\lambda$ at order $u^{2}$ if $\lambda=0$. They are given by

$$
\begin{aligned}
I_{0} & =-\frac{f_{2}(1)}{4 m \epsilon} \int d^{d-1} r \varphi(\boldsymbol{r}, 0) \partial_{\alpha} \partial_{\alpha} \varphi(\boldsymbol{r}, 0)+\ldots, \\
I_{1} & =\frac{1}{4 m \epsilon} \int_{0}^{1} d t f_{2}(t) \int d^{d-1} r \varphi(\boldsymbol{r}, 0) \partial_{\alpha} \partial_{\alpha} \varphi(\boldsymbol{r}, 0)+\ldots, \\
I_{2} & =\frac{1}{4 m \epsilon} \int_{1}^{\infty} d t f_{2}(t) \int d^{d-1} r \varphi(\boldsymbol{r}, 0) \partial_{\alpha} \partial_{\alpha} \varphi(\boldsymbol{r}, 0)+\ldots, \\
I_{3} & =\frac{f_{2}(1)}{4 m \epsilon} \int d^{d-1} r \varphi(\boldsymbol{r}, 0) \partial_{\alpha} \partial_{\alpha} \varphi(\boldsymbol{r}, 0)+\ldots,
\end{aligned}
$$

where the ellipses stand for other types of terms. Upon going over to the $\boldsymbol{p} z$ representation, we obtain the result

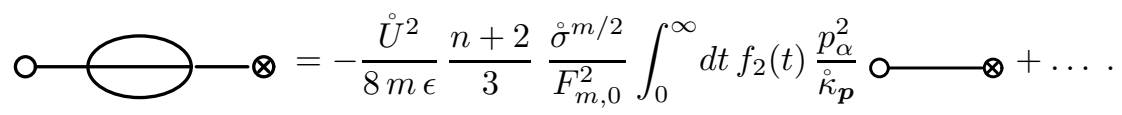

The explicitly displayed pole term must be absorbed by the renormalization of $\lambda$ according to Eq. (18). The expansion of the zero-loop graph $z_{1} @$ to first order in $\dot{\lambda}$ produces a contribution of the form $P_{\lambda}(u, \lambda=0, \epsilon) \stackrel{\circ}{\sigma}^{1 / 2}\left[\partial \hat{G}^{(1,1)} / \partial \grave{\lambda}\right]_{\grave{\lambda}=0}$. Its $u^{2} / \epsilon$ part must cancel the above pole. This is the case if we make the choice

$$
P_{\lambda}^{(2,-1)}(0)=-\frac{n+2}{3} \frac{\stackrel{\circ}{ }^{(m-1) / 2}}{8 m F_{m, 0}^{2}} \int_{0}^{\infty} d t f_{2}(t)=-\frac{n+2}{3} b_{m}\left[I^{(1)}(m)+I^{(2)}(m)\right],
$$

where $I^{(1)}(m)$ and $I^{(2)}(m)$ are the functions introduced in Appendix B [cf. Eqs. (B4)-(B6)].

\section{APPENDIX E: NUMERICAL EVALUATION OF INTEGRALS}

In this appendix we briefly explain how the numerical values of the integrals $J_{2,3}(m), j_{1}(m), I_{1}^{(1)}(m)$, and $I^{(2)}(m)$ presented in Table I were obtained.

\section{Numerical calculation of the integrals $J_{2,3}(m)$ and $j_{1}(m)$}

From its definition (49) it is clear that $J_{2,3}(m)$ is a single integral of a similar form $\int_{0}^{\infty} d v f(v)$ as the quantities $j_{\phi}(m), j_{\sigma}(m), j_{\rho}(m)$, and $j_{u}(m)$, which were previously introduced and numerically evaluated in Ref. 15 . The same applies to the integral $j_{1}(m)$ if we utilize its representation (77) in terms of $\Omega_{m, d^{*}}$. Unless $m$ takes the special values 2 or 6 , the integrands $f(v)$ involve differences of hypergeometric functions that grow exponentially as $v \rightarrow \infty$, while the $f(v)$ themselves decay as inverse powers of $v$ (cf. Appendix E of Ref. 15).

To cope with these difficulties, we proceeded as follows. Summing up the Taylor series expansions of the scaling functions $\Phi_{m, d^{*}}(v)$ and $\Omega_{m, d^{*}}(v)$ appearing in the integrands $f(v)$ gave reliable results as long as $v$ was not too large. Typically, this worked for all $v \leq v_{0}$ up to $v_{0} \simeq 10.7$. For larger values of $m$, even larger choices of $v_{0}$ were possible. 
We therefore worked with $v_{0}=10$ for both functions $\Phi_{m, d^{*}}(v)$ and $\Omega_{m, d^{*}}(v)$, and all $m$. Depending on whether $v$ was smaller or larger than this value of $v_{0}$, we relied on the Taylor summation and the asymptotic expansions of the functions $\Phi_{m, d^{*}}(v)$ and $\Omega_{m, d^{*}}(v)$ (see below).

In order to gain precision and speed, it proved useful to compute the required Taylor-series expansion coefficients in a recursive fashion. Starting from Eq. (12), one can easily show that the function $\Phi_{m, d^{*}}$ can be written as

$$
\Phi_{m, d^{*}}(v)=2^{-5-m} \pi^{-\frac{6+m}{4}}\left[\frac{8}{\Gamma\left(\frac{1}{2}+\frac{m}{4}\right)} \sum_{k=0}^{\infty} s_{k}\left(v^{4} / 64\right)-\frac{\sqrt{\pi}}{\Gamma\left(1+\frac{m}{4}\right)} v^{2} \sum_{k=0}^{\infty} \check{s}_{k}\left(v^{4} / 64\right)\right],
$$

where $s_{k}(x)$ and $\check{s}(x)$ satisfy the recursion relations

$$
s_{k+1}(x)=\frac{8 x s_{k}(x)}{(1+2 k)(2+m+4 k)}, \quad \check{s}_{k+1}(x)=\frac{4 x \check{s}_{k}(x)}{(1+k)(4+m+4 k)}, \quad s_{0}(x)=\check{s}_{0}(x)=1 .
$$

From Eq. (79) one easily derives the following analogous representation of $\Omega_{m, d^{*}}(v)$ :

$$
\Omega_{m, d^{*}}(v)=\frac{\sqrt{\pi}}{8 \Gamma(1+m / 4)} v^{m-2} \sum_{k=1}^{\infty} \frac{S_{k}\left(v^{4} / 64\right)}{m-2+4 k}-\frac{1}{\Gamma\left(\frac{1}{2}+\frac{m}{4}\right)} v^{m-4} \sum_{k=1}^{\infty} \frac{\check{S}_{k}\left(v^{4} / 64\right)}{m-4+4 k}+R(v)
$$

with

$$
S_{k+1}(x) \frac{4 x S_{k}(x)}{(1+k)(4+m+4 k)}, \quad \check{S}_{k+1}(x)=\frac{8 x \check{S}_{k}(x)}{(1+2 k)(2+m+4 k)}, \quad S_{0}(x)=\check{S}_{0}(x)=1,
$$

and

$$
R(v)=\frac{\sqrt{\pi} v^{m-2}}{8(m-2) \Gamma\left(1+\frac{m}{4}\right)}-\frac{8^{m / 2} \Gamma\left(\frac{1}{2}+\frac{m}{4}\right)}{32(m-2) \sin (m \pi / 4)}-\frac{v^{m-4}}{(m-4) \Gamma\left(\frac{1}{2}+\frac{m}{4}\right)} .
$$

The apparent singularities at $m=2$ and $m=4$ actually cancel, so that

$$
R(v)= \begin{cases}\frac{1}{2} v^{-2}+\frac{1}{8}\left(C_{E}-1\right)+\frac{1}{4} \ln (v / 2) & \text { for } m=2 . \\ \frac{1}{16} \pi^{1 / 2} v^{2}+\pi^{-1 / 2}\left[1-C_{E}+\ln \left(2 v^{-2}\right)\right] & \text { for } m=4 .\end{cases}
$$

In order to determine the values of the scaling functions for $v \geq v_{0}$, we utilized their asymptotic expansions. Those of the functions $\Phi_{m, d^{*}}$ can be found in Eqs. (A5) and (A6) of Ref. 15; those of the $\Omega_{m, d^{*}}(v)$ can be shown to read

$$
\Omega_{m, d^{*}}(v)=-\frac{16}{\sqrt{\pi}} v^{m-8} \sum_{k=0}^{\infty}\left(-\frac{64}{v^{4}}\right)^{k} \frac{\Gamma\left(\frac{3}{2}+k\right)}{\left(\frac{m}{4}-k-2\right) \Gamma\left(\frac{m}{4}-\frac{1}{2}-k\right)} .
$$

Note that if $m=2$ and $m=6$, this asymptotic series truncates at zeroth and first order, respectively, just as its analog for $\Phi_{m, d^{*}}(v)$ does for these choices of $m$.

Keeping the first three terms of the asymptotic expansions turned out to be sufficient. At $v=v_{0}=10$, the Taylor series of both $\Omega_{m, d^{*}}(v)$ and $\Phi_{m, d^{*}}(v)$ agreed with their respective (truncated) asymptotic series to within $10^{-4}$ to $10^{-7}$ percent.

Employing the above methods to compute the values of the integrands $f(v)$, the required integrals could be determined by straightforward numerical integration, giving the results for $J_{2,3}(m)$ and $j_{1}(m)$ presented in Table I. The numerical values found for $m=2$ and $m=6$ in this manner agree with the analytical results to the number of decimal digits retained or better. We also computed $j_{1}(m)$ by numerical evaluation of the double integral (76), obtaining results in conformity with those obtained by the above method.

\section{Calculation of $I^{(1)}(m), I^{(2)}(m)$, and related quantities}

The renormalization functions $C_{2}(u, \epsilon)$ and $P_{\lambda}^{(2,-1)}(0)$ involve the double integrals $I^{(1)}(m)$ and $I^{(2)}(m)$ defined by Eqs. (B5) and (B6). In the special cases $m=2$ and $m \rightarrow 6$, the required integrations can again be done analytically. The functions $\Psi_{m}^{(1)}$ and $\Psi_{m}^{(2)}$ [cf. Eqs. (B9) and (B10)] appearing in the integrands of $I^{(1)}(m)$ and 
$I^{(2)}(m)$ reduce to the simple expressions

$$
\Psi_{2}^{(1)}(y)=\Psi_{2}^{(2)}(y)=\frac{1}{2} \ln \frac{1+y^{1 / 2}}{\left|1-y^{1 / 2}\right|}
$$

and

$$
\Psi_{m}^{(1)}(y)=\Psi_{m}^{(2)}(y)=\frac{2}{6-m}\left[y^{-1 / 2}+O(6-m)\right],
$$

respectively. Upon substituting these results together with the simplified expressions for the scaling functions $\Phi_{2,5}(v)$ and $\Phi_{6,7}(v)$ into Eqs. (B5) and (B6), the integrations can be performed with the aid of MATHEMATicA ${ }^{61}$ to obtain the results (84)-(87). From them Eqs. (59) and (60) for $P_{\lambda}^{(2,-1)}(0)$ follow by insertion into Eq. (D7).

For other values of $m$, we performed the double integrals (B5) and (B6) numerically, employing Mathematica. ${ }^{61}$ In contrast to $\Phi_{m, d^{*}}(y)$, the functions $\Psi_{m}^{(1)}(y)$ and $\Psi_{m}^{(2)}(y)$ are not differences of functions that grow exponentially as $y \rightarrow \infty$. Therefore, their calculation poses no problem for MATHEMATICA even for large $y$. In the case of $\Phi_{m, d^{*}}(y)$ (and its powers appearing in the integrands), we simply let Mathematica evaluate its representation (12) in terms of hypergeometric functions if $y \leq y_{0} \simeq 8.5$, and utilized its asymptotic large- $y$ expansion for $y>y_{0}$. In each case we made sure that the values of the integrands the two methods yielded at the matching point $y=y_{0}$ were sufficiently close so that their difference could safely be neglected, given the limited number of decimal digits of our final numerical results.

The integrands of $I^{(1)}(m)$ and $I^{(2)}(m)$ have integrable singularities at $y=1$ and vary considerably in some parts of the two-dimensional integration regime. However, MathematicA's algorithm for numerical integration requires that the integrand does not vary too much. Letting us guide by two-dimensional plots of the integrands, we therefore divided the integration regimes into suitable rectangular subregions such that the algorithm was able to perform the integration over each of them without running into precision problems. We then added up the contributions from these rectangles to determine the integrals $I^{(1)}(m)$ and $I^{(2)}(m)$. The resulting numbers can be found in Table I.

The numerical values MATHEMATicA produces for $I^{(1)}(m)$ and $I^{(2)}(m)$ in the special cases $m=2$ and $m=6$ agree with the analytical results (84)-(87) to within $10^{-6}$ percent. However, since Mathematica automatically makes use of the fact that scaling functions such as $\Phi_{m, d^{*}}$ simplify considerably if $m=2$ and $m=6$, we must caution not to conclude that the same high numerical precision applies to our numerical results for other choices of $m$.

Let us also note that in the case $m=4, \Psi_{m}^{(1)}(y)$ and $\Psi_{m}^{(2)}(y)$ simplify to

and

$$
\Psi_{4}^{(1)}(y)=(1-y)^{-1 / 2} K[y /(y-1)]
$$

$$
\Psi_{4}^{(2)}(y)=\frac{2}{(y-1)^{1 / 2}+y^{1 / 2}} K\left(\left[\frac{(y-1)^{1 / 2}-y^{1 / 2}}{(y-1)^{1 / 2}+y^{1 / 2}}\right]^{2}\right),
$$

where $K(y)$ denotes a complete elliptic integral of the first kind.

A final remark concerns the sum $I^{(1)}(m)+I^{(2)}(m)$ appearing in $P_{\lambda}^{(2,-1)}(0)$. From Eq. (B4) we see that it is proportional to $\int_{0}^{\infty} d t f_{2}(t)$. The integration over $t$ is straightforward, producing a free propagator in $d^{*}-1$ dimensions. The result translates into

$$
\begin{aligned}
& I^{(1)}(m)+I^{(2)}(m) \\
& =\frac{1}{4} \int_{0}^{\infty} d R R^{1-m / 2} \int_{0}^{\infty} d \varrho \varrho^{m+1} \Phi_{m, d^{*}-1}\left(\varrho R^{-1 / 2}\right) \times \\
& \quad \times\left(R^{2}+1\right)^{-2} \Phi_{m, d^{*}}^{2}\left[\varrho\left(R^{2}+1\right)^{-1 / 4}\right] .
\end{aligned}
$$

Since the integrand on the right-hand side has less structure than those of the individual integrals $I^{(1)}(m)$ and $I^{(2)}(m)$, the form (E12) of the sum lends itself more easily to numerical evaluation via Mathematica. Our results for $P_{\lambda}^{(2,-1)}(0)$ presented in Eq. (61) were obtained in this fashion.
* On leave from: Institute of Solid State and Semiconductor Physics, 220072 Minsk, Belarus.

1 R. M. Hornreich, J. Magn. Magn. Mater. 15-18, 387 (1980).

${ }^{2}$ W. Selke, in Phase Transitions and Critical Phenomena, edited by C. Domb and J. L. Lebowitz (Academic, London, 1992), vol. 15, pp. 1-72.

${ }^{3}$ H. W. Diehl, Acta physica slovaca 52, 271 (2002), proc. of the 5th International Conference "Renormalization Group 2002", Tatranska Strba, High Tatra Mountains, Slovakia, March 10-16, 2002; cond-mat/0205284.

${ }^{4}$ W. Selke, Phys. Rep. 170, 213 (1988).

5 C. C. Becerra, Y. Shapira, N. F. Oliveira, Jr., and T. S. Chang, Phys. Rev. Lett. 44, 1692 (1980).
${ }^{6}$ C. S. O. Yokoi, M. D. Coutinho-Filho, and S. R. Salinas, Phys. Rev. B 29, 6341 (1964).

7 A. Zieba, M. Slota, and M. Kucharczyk, Phys. Rev. B 61, 3435 (2000).

8 C. C. Becerra, V. Bindilatti, and N. F. Oliveira, Jr., Phys. Rev. B 62, 8965 (2000).

9 M. E. Fisher and W. Selke, Phys. Rev. Lett. 44, 1502 (1980).

10 M. Pleimling and M. Henkel, Phys. Rev. Lett. 87, 125702/1 (2001), hep-th/0103194.

11 R. M. Hornreich, M. Luban, and S. Shtrikman, Phys. Rev. Lett. 35, 1678 (1975).

12 C. Mergulhão, Jr. and C. E. I. Carneiro, Phys. Rev. B 58, 6047 (1998). 
13 C. Mergulhão, Jr. and C. E. I. Carneiro, Phys. Rev. B 59, 13954 (1999).

14 H. W. Diehl and M. Shpot, Phys. Rev. B 62, 12338 (2000), cond-mat/0006007.

15 M. Shpot and H. W. Diehl, Nucl. Phys. B 612, 340 (2001), cond-mat/0106105.

${ }^{16}$ H. W. Diehl and M. Shpot, J. Phys. A 34, 9101 (2001), cond-mat/0106105.

17 H. W. Diehl and M. Shpot, J. Phys. A 35, 6249 (2002), cond-mat/0204267.

18 G. Gumbs, Phys. Rev. B 33, 6500 (1986).

19 K. Binder and H. L. Frisch, Eur. Phys. J. B 10, 71 (1999).

${ }^{20}$ H. L. Frisch, J. C. Kimball, and K. Binder, J. Phys.: Condens. Matter 12, 29 (2000)

21 M. Pleimling, Phys. Rev. B 65, 184406/1 (2002).

${ }^{22}$ In general, there may even be several different anisotropy exponents. If the isotropy is broken in both subspaces $\mathbb{R}^{m}$ and $\mathbb{R}^{d-m}$, then up to $d-1$ anisotropy exponents $\theta_{i} \neq 1$ are conceivable.

${ }^{23}$ H. W. Diehl and A. Gerwinski (2000), unpublished.

${ }^{24}$ A brief account of parts of this work has been given in Ref. 25. Note that Eq. (12) of this Letter contains a sign error, which is corrected by Eq. (58) of the present paper.

${ }^{25}$ H. W. Diehl, S. Rutkevich, and A. Gerwinski, J. Phys. A 36, L243 (2003).

26 Since $d^{*}(m)$ varies between $d^{*}(0)=4$ and $d^{*}(8)=8$, and $m$ can at most become equal to the surface dimension $d-1$ if $z$ is a $\beta$-direction, we have $m \leq d^{*}(7)-1=13 / 2$. Thus the largest permissible integer value of $m$ is $m=6$.

27 H. W. Diehl, M. Shpot, and R. K. P. Zia (2003), condmat/0307355.

28 H. W. Diehl, in Phase Transitions and Critical Phenomena, edited by C. Domb and J. L. Lebowitz (Academic, London, 1986), vol. 10, pp. 75-267.

${ }^{29}$ H. W. Diehl and H. K. Janssen, Phys. Rev. A 45, 7145 (1992).

30 H. W. Diehl, Phys. Rev. B 49, 2846 (1994).

31 As usual, these hold inside of averages (i.e., in an "operator sense") and follow from the contributions localized on the surface of the equations of motion; see, e.g., Refs. 28, 29, and 30 .

${ }^{32}$ H. W. Diehl, Int. J. Mod. Phys. B 11, 3503 (1997), condmat/9610143.

33 In order to compare with the $m=0$ results of Refs. 38, 39 , and 28 , one should take into account that a different normalization of the coupling constant was employed in these references, which implies the following correspondence between their $Z$-factors $Z_{\mathrm{DD}}(u)$ and ours here: $Z_{\mathrm{DD}}(u / 2)=Z(u)$.

34 In Ref. 14, explicit results for the scaling function $\Xi_{m, d}(v)$ in terms of generalized hypergeometric functions are given which generalize Eq. (51) to the case $d \neq d^{*}(m)$; cf. its equation (A3). Integral representations of $\Xi_{m, d}(v)$ can be found in this reference as well.

${ }^{35}$ In the continuous symmetry case, a surface phase with homogeneous long-range order above the bulk transition temperature is not expected unless the surface dimension $d-1$ exceeds the usual lower bulk critical dimension $d_{*}=2$ of the $O(n)$ vector model.

${ }^{36}$ H. W. Diehl and S. Dietrich, Phys. Lett. 80A, 408 (1980).

37 H. W. Diehl and S. Dietrich, Z. Phys. B 42, 65 (1981), erratum: 43, 281 (1981).

38 H. W. Diehl and S. Dietrich, Phys. Rev. B 24, 2878 (1981).

39 H. W. Diehl and S. Dietrich, Z. Phys. B 50, 117 (1983).
40 T. Garel and P. Pfeuty, J. Phys. C 9, L246 (1976).

41 M. E. Fisher, in Critical Phenomena, edited by F. J. W. Hahne (Springer-Verlag, Berlin, 1983), vol. 186 of Lecture Notes in Physics, pp. 1-139.

42 For background on dangerous irrelevant variables, see, e.g., Appendix D of Ref. 41.

43 These are analogs of the nonuniversal amplitudes $E_{c}^{*}$ and $E_{h_{1}}^{*}$ utilized in Eqs. (3.105b) and (3.105d) of Ref. 28. In the present $m>0$ case, they do not only depend on $u$, the initial value of the running coupling constant $\bar{u}$, but also on $\lambda$.

44 Strictly speaking, this is guaranteed at this stage only for sufficiently small $\epsilon>0$ because the $\epsilon$ dependent terms of the exponent $1+O(\epsilon)$ appearing in $(1 / \bar{c})^{M(1+O(\epsilon))}$ can then be trusted to be positive. However, from the well-studied $m=0$ case we know that this exponent is proportional to the difference $\beta_{1}^{\text {ord }}-\beta_{1}^{\text {sp }}$ of the surface critical exponents $\beta_{1}^{\text {ord/sp }}$ of the ordinary and special CP transitions, respectively. This difference is positive and expected to be so even in the $m>0$ case.

45 In the special case $\rho=0, \xi_{l 2}$ and $\xi_{l 4}$ correspond to correlation lengths for the $d-m$ "hard" and $m$ "easy" directions. In the general case $\rho \neq 0$ and $g_{\tau} \neq 0$, this is no longer true, irrespective of whether such correlation lengths are defined via second moments of the two-point function $G^{(2,0)}$ or in terms of its exponential decay along these directions ("true correlation lengths"). Unlike our $\xi_{l 2}$ and $\xi_{l 4}$, which are independent of the scaling field $\sigma$, such correlation lengths depend on both relevant scaling fields $g_{\tau}$ and $\sigma$.

46 S. Dietrich and H. W. Diehl, Z. Phys. B 43, 315 (1981).

47 H. W. Diehl, S. Dietrich, and E. Eisenriegler, Phys. Rev. B 27, 2937 (1983).

48 T. W. Burkhardt and J. L. Cardy, J. Phys. A 20, L233 (1987).

49 T. W. Burkhardt and H. W. Diehl, Phys. Rev. B 50, 3894 (1994).

50 In other words, the analogous bulk model with homogeneous values of $\stackrel{\circ}{\tau}=\stackrel{\circ}{\tau}_{2}$ and $\stackrel{\circ}{\rho}=\stackrel{\circ}{\rho}_{2}$ and the Hamiltonian $\mathcal{H}=\int_{\mathbb{R}^{d}} \mathcal{L}_{\mathrm{b}}(\boldsymbol{x} ; \stackrel{\circ}{\tau}, \stackrel{\circ}{\rho}) d V$ should have these properties.

51 E. Eisenriegler and M. Stapper, Phys. Rev. B 50, 10009 (1994).

52 A natural way to define the above-mentioned surface index $\eta_{l 4, \perp}$ would be to write the exponent $\left(2-\eta_{\perp}\right) / \theta$ in the second line on the right-hand side of Eq. (134) as $2-\eta_{l 4, \perp}$.

${ }^{53}$ K. Binder, in Phase Transitions and Critical Phenomena, edited by C. Domb and J. L. Lebowitz (Academic, London, 1983), vol. 8, pp. 1-144.

54 J. S. Reeve and A. J. Guttmann, Phys. Rev. Lett. 45, 1581 (1980).

55 M. C. Barbosa, Physica B 215, 286 (1995).

56 W. Selke, Solid State Comm. 27, 1417 (1978).

57 W. Selke, J. Phys. C 13, L261 (1980).

${ }^{58}$ H. W. Diehl, A. Gerwinski, and S. Rutkevich (2002), unpublished.

59 Test functions are normally required to have strong smoothness properties, e.g., to have continuous partial derivatives of arbitrary order and to decay faster than any power as any of its arguments approaches $\pm \infty$. The amputated legs we are concerned with do not possess such strong properties. For example, the amputated leg $G_{\mathrm{D}}\left(\boldsymbol{x}_{1}, \boldsymbol{x}\right)$ and its Fourier transform $\hat{G}_{\mathrm{D}}\left(\boldsymbol{p} ; z_{1}, z\right)$ depend on $\left|z_{1}-z\right|$ and $\left|z_{1}+z\right|$, so that sufficiently high $z$ derivatives produce (derivatives of) $\delta\left(z_{1} \pm z\right)$. However, one can easily see that 
the actions of the distributions in question on the external legs are well defined. The formulae derived for genuine test functions $\varphi$ and $\psi$ apply, if we consider the cumulants in the $\boldsymbol{p} z$ representation as functions of the $z$ coordinates $z_{1}$ and $z_{2}$ of their external points off the surface. Amputated legs whose external points are located on the surface, such as functions of $z$.

With regard to the coordinates $\boldsymbol{r}$ parallel to the surface the situation is even simpler and completely analogous to the bulk case. Owing to the translational invariance we have parallel to the surface, the required integrals over $r$ variables can be rewritten as convolutions. Such convolutions of well-defined generalized functions of $\boldsymbol{r}$ variables (i.e., of the amputated graph with the two amputated legs) are well-defined, becoming simple products in $\boldsymbol{p}$ space.

${ }^{60}$ I. M. Gel'fand and G. E. Shilov, Generalized Functions (Academic, New York and London, 1964), vol. 1.

61 MathematicA, version 3.0, a product of Wolfram Research. 
$\otimes-1-1-1$ 


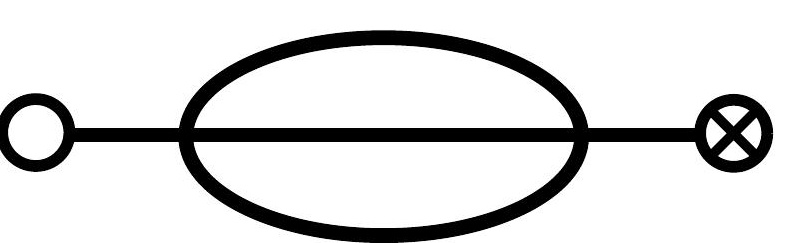

